\title{
Work Environment and Psychosocial Factors Affecting Physical Activity among Taiwanese Information Technology Professionals
}

\author{
by \\ Yun-Ping Lin \\ A dissertation submitted in partial fulfillment \\ of the requirements for the degree of \\ Doctor of Philosophy \\ (Nursing) \\ in The University of Michigan \\ 2011
}

Doctoral Committee:

Professor Janet L. Larson, Chair

Professor Dee W. Edington

Assistant Professor Tsui-Sui Annie Kao

Assistant Professor Marjorie C. McCullagh 
(C) Yun-Ping Lin 2011 


\section{DEDICATION}

To my beloved parents and mother-in-law

Thank you for your endless love and support!

To the love of my life, my husband, Philip and daughter, Ivy

Thank you for always believing in me and pushing me to do my best!

I could not have done it without you!

To my dear brothers, sisters- and brothers-in-law

Thank you for your support, help, and encouragement along the way! 


\section{ACKNOWLEDGMENTS}

This dissertation would not have been possible without the support of many people. First and foremost are the members of my committee. I am deeply grateful to my chair, Dr. Janet Larson, for her extraordinary guidance, inspiration, patience, and enthusiasm. Her divine wisdom has helped me become a better researcher and writer, and she has been an excellent role model for me. Dr. Annie Kao's timely statistical guidance has provided reassurance to the quality of the data analysis. She has been a valued mentor during this journey. Dr. Marjorie McCullagh's expertise in occupational and environmental health nursing has been a great source of knowledge, support, and encouragement. Dr. Dee Edington, a truly expert in the field of individual health promotion and worksite wellness, provided insightful comments on the proposal development stage and a supportive environment framework to help me understand my findings in the workplace context. My deepest gratitude to these dedicated scholars.

I extend my sincere appreciation to Dr. Huey-Ming Tzeng for her generous and timely assistance with the instrument translation by serving as a referee. Her wise advice and experience have helped guide me through this process. In addition, I wish to expressly thank Drs. Oisaeng Hong and Kimberlee Gretebeck, as my academic advisors, for their guidance in the formulation of my program and research plans. I am very grateful for their investment, support, and encouragement along the way.

I would like to thank the following individuals for their statistical support: Dr. Amiram Vinokur from the Institute for Social Research (ISR) and Ms. Laura Klem and 
Mr. Brady West at the University of Michigan Center for Statistical Consultation and Research (CSCAR). I would also like to recognize the financial support of this work from the American Association of Occupational Health Nurses (AAOHN) Foundation, the Midwest Nursing Research Society (MNRS), the Sigma Theta Tau International, Rho Chapter, and the University of Michigan Rackham Graduate Student Research Grant. Each of their contributions is sincerely appreciated.

My heartfelt gratitude goes to the three IT companies that agreed to participate in this project. A special thank you to the participants who gave their time, and the wonderful people who worked there and supported my project to make this dissertation possible. Lastly, my sincere thanks to all my dear professors, friends, and classmates who supported, encouraged, and cared for me during this journey. Thank you for sharing your time, energy, and knowledge with me. 


\section{TABLE OF CONTENTS}

$\begin{array}{ll}\text { DEDICATION } & \text { ii }\end{array}$

ACKNOWLEDGMENTS

LIST OF FIGURES viii

LIST OF TABLES $\quad$ ix

LIST OF APPENDICES $\quad$ xi

ABSTRACT $\quad$ xii

CHAPTER

1. Introduction 1

Statement of the Problem 4

Structure of the Dissertation $\quad 5$

$\begin{array}{lr}\text { Theoretical Framework } & 6\end{array}$

2. Work Environment and Psychosocial Determinants of Physical Activity among White-Collar Workers: A Review of the Literature 17

$\begin{array}{ll}\text { Abstract } & 17\end{array}$

$\begin{array}{ll}\text { Introduction } & 18\end{array}$

$\begin{array}{ll}\text { Methods } & 20\end{array}$

$\begin{array}{ll}\text { Results } & 20\end{array}$

$\begin{array}{ll}\text { Discussion } & 29\end{array}$ 
3. Reliability and Validity of the Chinese Version of the Perceived Workplace Environment Scale in Taiwanese Information Technology Professionals

Abstract

Introduction

Methods

Instrument Translation

Description of the Other Measure

Data Analysis

Results

Discussion

4. Work Environment and Psychosocial Factors Affecting Physical Activity among Taiwanese Information Technology Professionals

Abstract

Introduction

Methods

Overview of Analytic Procedures

Results

Discussion

Study Strengths and Limitations

Implications for Practice and Policy

Conclusions

5. Conclusions

Summary

Implications 
APPENDICES 


\section{LIST OF FIGURES}

\section{FIGURE}

1.1 Hypothesized workplace physical activity model

3.1 Factor structure and standardized factor loadings on the Chinese version of the perceived workplace environment scale items

4.1 Hypothesized workplace physical activity model

4.2. Structural equation model of the effects of work environment and psychosocial factors on physical activity

4.3. Gender comparison structural equation model of the effects of work environment and psychosocial factors on physical activity 


\section{LIST OF TABLES}

\section{TABLE}

2.1 Characteristics and Main Findings of Studies Examining Relationships of Work Environment Determinants with Physical Activity among White-Collar Workers 33

2.2 Characteristics and Main Findings of Studies Examining Relationships of Psychosocial Determinants with Physical Activity among White-Collar Workers 40

2.3 Association of Job Strain Variables with Physical Activity among White-Collar Workers

2.4 Association of Psychosocial Variables with Physical Activity among White-Collar Workers

3.1 Items of the Original Perceived Workplace Environment Scale (PWES)

3.2 Chinese Version of the Perceived Workplace Environment Scale (PWES-C) 64

3.3 知覺職場身體活動環境量表中文版

3.4 Descriptive Statistics and Distribution of Data for the PWES-C and IPAQ-Taiwan $(\mathrm{N}=575)$

3.5 Fit Statistics of the PWES-C from Confirmatory Factor Analysis

3.6 Internal Consistency Reliability of the PWES-C

4.1 Descriptive Statistics and Internal Consistency Reliabilities for the Scales

4.2 Factor Loadings and Error for the Measured Variables (Indicators) of Latent Factors Used in the Confirmatory Factor Analysis Measurement Model

4.3 Sample Characteristics by Gender 
4.5 Means, Standard Deviations, and Zero-Order Correlations Among Variables Included in the Model

4.6 Fit Statistics of the Structural Equation Model of the Effects of Work Environment and Psychosocial Factors on Physical Activity for the Entire Sample

4.7 Participant Recommendations for Promoting Physical Activity for IT Professionals in Their Company $(\mathrm{n}=175)$ 


\section{LIST OF APPENDICES}

APPENDICES

A. The English Version of the Questionnaire

B. The Chinese Version of the Questionnaire 


\title{
ABSTRACT \\ Work Environment and Psychosocial Factors Affecting Physical Activity among Taiwanese Information Technology Professionals
}

\author{
by \\ Yun-Ping Lin
}

Chair: Janet L. Larson

Information technology (IT) professionals are typically sedentary and little is known about factors that influence their physical activity. Research has been limited by a lack of validated measures of workplace physical activity environments. PURPOSES: The purposes of this study were to (a) examine psychometric properties of a newly translated instrument for measuring workplace environments and (b) examine relationships among work environment (supportive workplace environments and job strain), psychosocial factors (outcome expectations and self-efficacy for physical activity), and physical activity in Taiwanese IT professionals. METHODS: This research was guided by a hypothesized model using Social Cognitive Theory, incorporating variables from the demand/control model. This was a cross-sectional survey of 576 IT professionals (467 men and 109 women; $M=33.7$ years, $S D=6.08$ ) from three IT companies in Taiwan. Three instruments were translated into Chinese using a modified committee approach. Participants completed a Chinese questionnaire that included the 
International Physical Activity Questionnaire-Taiwan long form, Perceived Workplace Environment Scale (PWES-C), Psychological Job Demands and Job Control Scale, Scale of Exercise Self-Efficacy, Positive and Negative Outcome Expectations Scale (PNOESC), and Historical Physical Activity Questionnaire (HPAQ-C). Structural equation modeling (SEM) was used to test the model. RESULTS: Psychometric properties of the PWES-C demonstrated satisfactory internal consistency reliability, content and construct validity. SEM analyses showed that (a) higher levels of physical activity were associated with higher perceptions of supportive workplace environments, positive outcome expectations, and self-efficacy; (b) self-efficacy partially mediated the effects of supportive workplace environments on physical activity; (c) job strain had an indirect effect on physical activity through self-efficacy; (d) the final model accounted for $31 \%$ of the variance in physical activity; and (e) the effect of gender on our findings was not substantial in multigroup analyses. CONCLUSIONS: The PWES-C is a reliable and valid measure of perceived workplace environment in Taiwanese IT professionals. Both work environment and psychosocial factors are important. Interventions directed toward increasing individuals' confidence in their ability to overcome barriers to physical activity and positive expected outcomes of physical activity in the context of supportive workplace environments may be useful. Study limitations, implications and future directions are discussed. 


\section{CHAPTER 1}

\section{Introduction}

Taiwan Healthy People 2020 demonstrated links between the modern living and working environments, a decrease in physical activity, sedentary behaviors and negative health consequences and the economic burden in Taiwan (Chen et al., 2008). This

document indicates that the least physically active groups are among those aged 25 to 44 (inadequate physical activity: 40-43\%) and white-collar workers (42\%). In response, Taiwan Healthy People 2020 objectives relating to physical activity include increasing the proportion of adults aged 18-65 who engage in adequate physical activity $(64-66 \%$ by $2012 ; 66-68 \%$ by $2016 ; 67-70 \%$ by 2020$)$, and increasing the proportion of workplaces that provide physical activity and fitness classes at least 2 days per week. In addition, an ecological approach is proposed to develop specific strategies to achieve these objectives for physical activity (Chen et al.).

Despite an increased emphasis on the health benefits of regular physical activity, a recent Internet survey revealed that Taiwanese information technology (IT) workers are the least active working population, with approximately $70 \%$ considering themselves to be physically inactive (Taiwan 1111 Job Bank, 2007a). A study of 106 Taiwanese IT workers showed that only $4 \%$ engaged in leisure-time physical activity at least three times per week (Hsu \& Huang, 2001), compared to $30 \%$ of all Taiwanese adults (Ku, Fox, McKenna, \& Peng, 2006). Among these IT workers, results of their physical fitness tests 
were substandard; in particular, $35 \%$ of the participants were overweight or obese, and another 77\% had unsatisfactory cardiorespiratory endurance and leg muscle strength (Hsu \& Huang).

Over the last three decades, the IT services have emerged as one of the most important industries in the global economy. This industry is also regarded as a prime force in Taiwan's economic growth, and the employment of IT workers increased from 65,499 in 2006 to 80,748 in 2009, and is expected to grow (Taiwan Science and Technology Advisory Group, 2007). The ever-increasing global market competition, rapid technological advances, and tight deadlines have made the IT profession a very demanding and stressful job requiring long hours that contribute to the sedentariness of IT workers (Taiwan 1111 Job Bank, 2007b; Taiwan Council of Labor Affairs, 2007). Epidemiologic studies have documented the adverse health effects of sedentary work behaviors. Morris, Heady, Raffle, Roberts, and Parks (1953a, 1953b) found that more active bus conductors and postmen had lower rates of coronary heart disease than sedentary bus drivers and mail sorters in England. Taylor et al. (1962) concluded that U.S. railroad workers in sedentary jobs had more coronary heart disease than those in jobs requiring moderate to heavy physical activity. Current research indicates that sitting (the most common sedentary behavior) is hazardous to health, and too much sitting is different from lack of physical activity. Prolonged sitting is harmful to cardiovascular and metabolic effects (e.g., waist circumference, 2-hour plasma glucose, triglycerides, and high-density lipoprotein [HDL] cholesterol), which are independent of moderate to vigorous physical activity and waist circumference. However, frequent light-intensity physical activity or breaks in sedentary time (even 5 seconds) throughout the day are 
beneficially associated with metabolic biomarkers and overall energy expenditure (Hamilton, Hamilton, \& Zderic, 2007; Hamilton, Healy, Dunstan, Zderic, \& Owen, 2008).

The economic burden of physical inactivity has been estimated in studies from the U.S., the U.K., and Canada (Allender, Foster, Scarborough, \& Rayner, 2007; Anderson et al., 2005; Katzmarzyk, Gledhill, \& Shephard, 2000; Pronk, Goodman, O’Connor, \& Martinson, 1999). For example, inactive people incurred 24\% higher health care costs than their active counterparts (Pratt, Macera, \& Wang, 2000), and 4.7\% of health care costs could be decreased by each additional day of physical activity per week (Pronk et al.). Workers who were moderately active (1-2 times/week; $\geq 20$ minutes/time) and very active ( $\geq 3$ times/week; $\geq 20$ minutes/time) had lower health care costs (approximately $\$ 250 /$ year) than sedentary ( $<1$ time/week) workers (Wang, McDonald, Champagne, \& Edington, 2004).

Taiwan government agencies and academia have sought to improve worker health and productivity through the implementation of workplace physical activity or fitness programs (Fan \& Yen, 2008); however, the effects of these programs (conducted in Taiwan) on increasing physical activity or fitness have been mixed (Chen \& Ling, 2004; Hsu \& Huang, 2001; Huang, Kao, Chen, \& Hsu, 2003; Jwo, Tong, \& Yen, 1992; Li, Tseng, Tseng, \& Lee, 2006; Wang \& Chang, 2006). In spite of some methodological flaws (e.g., fidelity of implementation and contamination concerns), three theory-based programs using quasi-experimental designs yielded short-term increases in worker physical activity (Huang et al.; Wang \& Chang) or fitness (Jwo et al.). But it is not clear how efficacious these programs are with IT workers (Note: samples in Wang and Chang's study were high-tech workers including IT workers; the others were office 
workers). Furthermore, these programs have tended to operate on a personal (individual) level (e.g., education and/or stretching exercises during breaks) despite recent support for the potential impact of broad policy and environmental changes to promote physical activity (Brug, Oenema, \& Ferreira, 2005; Engbers, van Poppel, Chin, \& van Mechelen, 2005; Foster \& Hillsdon, 2004; Kahn et al., 2002; Kremers et al., 2006; Matson-Koffman, Brownstein, Neiner, \& Greaney, 2005; McLeroy, Bibeau, Steckler, \& Glanz, 1988; Plotnikoff, Prodaniuk, Fein, \& Milton, 2005; Sallis, Bauman, \& Pratt, 1998; Spence \& Lee, 2003).

Overall, only a few published workplace physical activity studies conducted in Taiwan have examined the relationships between physical activity or exercise and its determinants (Chen \& Chang, 2004; Kao, Lu, \& Huang, 2002; Lee, Huang, \& Kao, 2005). Each of these studies focused primarily on psychosocial factors at the personal level, with the exception of one recent unpublished dissertation study that included the influence of work environment on worker physical activity (Chang, 2007). None of these studies were conducted with IT workers. Little is known about the nature of physical activity and its determinants among Taiwanese IT workers. To promote the health of this working population, it is important to identify those factors that influence their physical activity as a first step toward designing effective interventions.

\section{Statement of the Problem}

Epidemiologic evidence suggests that workers whose jobs required long hours of sitting had two times the increased risk of cardiovascular disease than those whose jobs required physical effort (Hamilton et al., 2008). IT workers are typically inactive due to the sedentary nature of their jobs and pastimes (Taiwan 1111 Job Bank, 2007a, 2007b). 
To our knowledge, no published studies have explored the specific nature of physical activity (e.g., different types of physical activity) and its determinants among IT workers. Although much of the recent literature suggests environmental influences on physical activity, only a few studies (Dodson, Lovegreen, Elliott, Haire-Joshu, \& Brownson, 2008; Lucove, Huston, \& Evenson, 2007; Prodaniuk, Plotnikoff, Spence, \& Wilson, 2004) have examined this relationship in workers. Even fewer (Prodaniuk et al.) have focused specifically on multiple dimensions of the workplace environment. This is most likely due to the challenges of operationalizing the environment constructs and the lack of psychometrically sound instruments (Sallis \& Owen, 2002).

\section{Structure of the Dissertation}

This is a manuscript-style dissertation. Three manuscript-style papers are presented in the next three chapters. Chapter 2 presents a critical review of the empirical research on the work environment and psychosocial determinants of physical activity among white-collar workers and provides recommendations for future research. Chapter 3 describes the translation process of the PWES from English into Chinese, as well as examines the distribution of the data, internal consistency reliability, and construct validity of the Chinese version. Chapter 4 presents the results of the present investigation designed to examine the relationships among work environment (i.e., supportive workplace environment and job strain), psychosocial factors (i.e., outcome expectations and self-efficacy for physical activity), and physical activity in Taiwanese IT professionals. The final chapter (Chapter 5) concludes with a summary of the main findings and implications of the three papers and some directions for future work. 


\section{Theoretical Framework}

Based on the review of previous studies, a hypothesized workplace physical activity model (see Figure 1.1) was developed using Social Cognitive Theory (i.e., supportive workplace environments, outcome expectations, and self-efficacy for physical activity) (Bandura, 1986), incorporating variables from the demand/control model (i.e., job strain; the ratio of job demands to job control) (Karasek, 1979). This approach aligns with the view that "Synergy may be achieved by taking the most promising concepts from each model and integrating them for use with specific populations" (Baranowski, Cullen, Nicklas, Thompson, \& Baranowski, 2003). This also addresses the recommendation to examine how environmental and psychosocial variables interact in their effects on physical activity (Marcus et al., 2006).

\section{Environmental Influences (Work Environment)}

Personal Factors (Psychosocial)
Behavioral Outcome

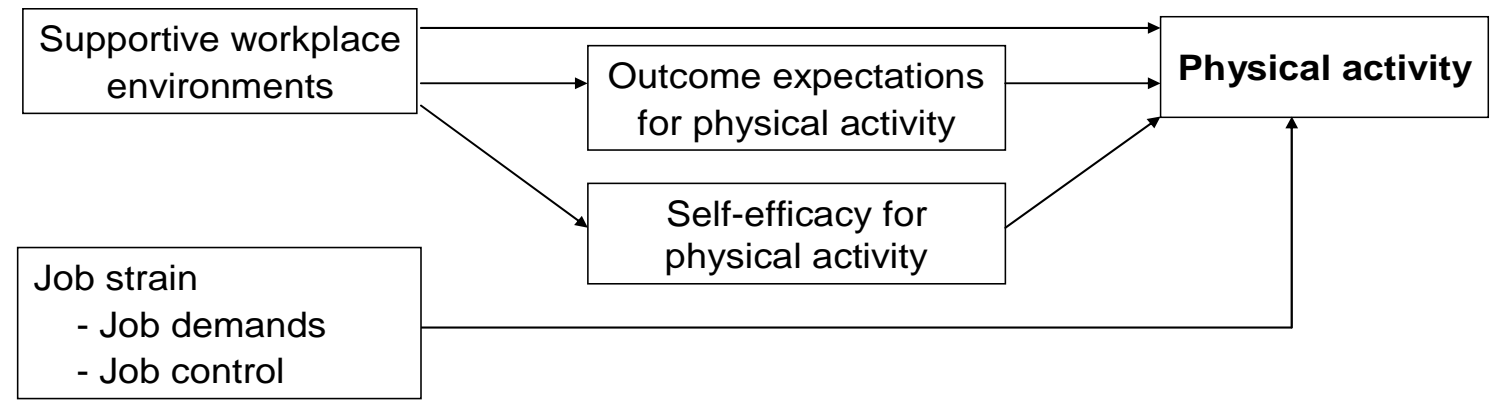

Figure 1.1. Hypothesized workplace physical activity model.

For purposes of this model, the variable of physical activity refers to any physical activity that is performed at work, in and around the home, while traveling from place to place, and for the purpose of recreation, exercise or sport for at least 10 minutes at a time 
(Craig et al., 2003). Supportive workplace environments refer to workers' perceptions of their workplace environments that support physical activity (Prodaniuk et al., 2004). Job strain is stress which occurs when individuals face high psychological job demands while simultaneously having low control over their work; job strain is thus defined as the ratio of job demands to job control (Karasek, 1979). Psychological job demands are psychological stressors related to workload, work pace, and organizational constraints on completing work. Job control is workers' perceptions of control over their work, reflecting the extent to which they use intellectual skills and make decisions (Karasek). Outcome expectations for physical activity are an individual's judgment of the likely consequences of engaging in regular physical activity (Bandura, 1986). Self-efficacy for physical activity is an individual's judgment of his/her confidence to engage in regular physical activity despite various barriers (Bandura).

In the model, physical activity behavior is posited to be the outcome of a simultaneous influence of conscious and unconscious processes (Kremers et al., 2006). Supportive workplace environments are hypothesized to affect physical activity both directly and indirectly through the mediating role of outcome expectations and selfefficacy for physical activity. The indirect effect reflects relatively conscious influence of supportive workplace environments on physical activity. The direct effect reflects relatively automatic or unconscious influence of supportive workplace environments on physical activity. Job strain is hypothesized to affect physical activity directly.

\section{Social Cognitive Theory}

Bandura's Social Cognitive Theory postulates that a person performs a behavior and that this behavior has certain outcomes. Two kinds of determinants emerge from this 
theory: (a) self efficacy is a belief concerning one's ability to perform a specific behavior in a particular situation; (b) outcome expectations are beliefs which ask whether performing a specific behavior will produce certain outcomes (Bandura, 1997; Strecher et al., 1986).

Self-efficacy plays an important role in changing one's behavior, and personal health behavior change would be easy if there were no obstacles or barriers to overcome. People with high self-efficacy tend to view barriers as surmountable, whereas those with low self-efficacy tend to give up or reduce their effort. In addition, self-efficacy beliefs are hypothesized to influence the outcomes that people expect their efforts to produce. People with high self-efficacy expect to produce desired outcomes, whereas those with low self-efficacy expect their efforts to produce poor outcomes (Bandura, 2004).

Health behaviors are also influenced by the outcomes that people anticipate their performances to occur. Outcome expectations include three major forms: (a) physical outcomes are about pleasurable and aversive effects resulting from performing a behavior; (b) social effects are about a behavior leading to opportunities for social interaction and gaining social approval as well as disapproval; and (c) self-evaluative reactions to one's behavior are about the feelings of self-satisfaction and self-worth as well as selfdissatisfaction resulting from performing a behavior. Each form contains positive and negative outcome expectations, with the positive ones serving as incentives and the negative ones as disincentives (Bandura, 1997, 2004).

Social Cognitive Theory posits that behavior is dynamically and continuously interacting with both personal factors and environmental influences (triadic reciprocal determinism) (Bandura, 1986). It is similar to ecological models because it shares the 
same perspective that the environment can affect one's behavior (Spence \& Lee, 2003). In the proposed model of workplace physical activity (see Figure 1.1), environmental influences include two variables: supportive workplace environments and job strain. Job strain is discussed in the following section of the demand/control model. Supportive workplace environments were defined based on an ecological workplace physical activity model (Plotnikoff et al., 2005). The model consists of six workplace environment dimensions: (a) individual (i.e., factors in the workplace influencing worker characteristics regarding physical activity, such as knowledge, attitude, and skills); (b) social (i.e., influence of the corporate culture, social relationships, peer and supervisor attitudes regarding worker physical activity); (c) organizational (i.e., how the organizational structure impacts worker physical activity: infrastructure, leadership, and desire to promote physical activity); (d) community (i.e., how the workplace interacts or partners with, or uses other organizations, community, or government resources that may promote worker physical activity); (e) policy (i.e., the workplace's policies regarding worker physical activity); and (f) physical environment (i.e., the workplace's physical environment as it affects worker physical activity, such as buildings, grounds, and surrounding areas) (Plotnikoff et al.; Prodaniuk et al., 2004).

Social Cognitive Theory provides both determinants of health behavior and principles to inform and guide the development of interventions to motivate people to adapt and maintain a health behavior (Bandura, 2004). Importantly, there have been recent calls to examine the potential mediation effects of psychosocial factors on the relationship between environmental factors and physical activity, in an attempt to guide development of interventions (Baranowski et al., 2003; Brug et al., 2005; Kremers et al., 
2006; Prodaniuk et al., 2004; Sallis \& Owen, 2002; Spence \& Lee, 2003). Yet surprisingly, except for one study (Plotnikoff et al., 2005; Prodaniuk et al.), there is a dearth of research examining this mediation effect on worker physical activity.

\section{Demand/Control Model}

Karasek's (1979) demand/control model is also termed the job strain model. Job strain results from high psychological job demands combined with low decision latitude (job control). Accordingly, Karasek posited that job strain is equivalent to the excess of job demands over job control (i.e., ratio of job demands to job control). Job demands are the psychological demands of work ("how hard you work") related to workload, work pace, organizational constraints on completing work, and conflicting demands. Examples include how many projects are due this month and the proximity of deadlines. Time pressure can impose high mental workload. Decision latitude (job control) refers to workers' ability to control the performance of their own jobs, which is measured by skill discretion (task variety) and decision authority (autonomy). Skill discretion and decision authority are highly correlated because workers with high levels of skills are more likely to control the particular skills to complete their work (Karasek et al., 1998; Karasek \& Theorell, 1990).

High job demands may cause anxiety about job performance and time pressure on completing work. However, the model hypothesizes that the impact of excessive job demands may be buffered by job control if workers are allowed to participate in the decision-making process (decision authority) and can use a variety of skills to do their jobs (skill discretion) (Cooper, Dewe, \& O'Driscoll, 2001). Karasek and Theorell (1990) also hypothesized that workers with decision latitude over their tasks will decrease their 
stress but increase learning, whereas psychological demands will both increase stress and learning. Yet, if these demands are associated with lack of control (i.e., not positive challenges), they will not increase learning. The authors emphasized that it is vital to examine job demands and job control in the workplace separately and then review their combined effects. In addition, low job control and high job demands can be used as independent indicators of job strain (Karasek et al., 1998).

In summary, the theoretical framework proposed here suggests that work environment factors (i.e., supportive workplace environments and job strain) may influence worker physical activity. The effects of work environment on physical activity, one's perceptions of workplace environments to support physical activity and one's control over his/her work when facing high psychological job demands, may facilitate or inhibit physical activity. Psychosocial variables such as outcome expectations and selfefficacy for physical activity that may affect physical activity are hypothesized to have the mediation effects on the relationship between supportive workplace environments and physical activity. Through a better understanding of workers' work environment and psychosocial determinants, ways may be found to increase their physical activity, and eventually resulting in a healthy workforce and high-performance workplace. 


\section{References}

Allender, S., Foster, C., Scarborough, P., \& Rayner, M. (2007). The burden of physical activity-related ill health in the UK. Journal of Epidemiology and Community Health, 61(4), 344-348.

Anderson, L. H., Martinson, B. C., Crain, A. L., Pronk, N. P., Whitebird, R. R., O"Connor, P. J., et al. (2005). Health care charges associated with physical inactivity, overweight, and obesity. Preventing Chronic Disease, 2(4), 1-12.

Bandura, A. (1986). Social foundations of thought and action: A social cognitive theory. Englewood Cliffs, N.J.: Prentice-Hall.

Bandura, A. (1997). Self-efficacy: The exercise of control. New York: W.H. Freeman.

Bandura, A. (2004). Health promotion by social cognitive means. Health Education \& Behavior, 31(2), 143-164.

Baranowski, T., Cullen, K. W., Nicklas, T., Thompson, D., \& Baranowski, J. (2003). Are current health behavioral change model helpful in guiding prevention of weight gain efforts? Obesity Research, 11 Suppl, 23S-43S.

Brug J., Oenema, A., \& Ferreira, I. (2005). Theory, evidence and intervention mapping to improve behavior nutrition and physical activity interventions. International Journal of Behavioral Nutrition and Physical Activity, 2(1), 2.

Chang, S. H. (2007). Physical activity and its determinants among workers -- A case of traditional industry's employee in Taiwan. Unpublished doctoral dissertation, National Taiwan University, Taipei. (in Chinese)

Chen, C. M., \& Chang, M. (2004). Exercise behavior and related factors in career women -- The case of a bank in Taipei City. Journal of Nursing Research, 12(3), 180-189.

Chen, C. M., \& Ling, T. S. (2004). Evaluation of physical health fitness promotion strategy. Chinese Journal of Occupational Medicine, 11(4), 209-215. (in Chinese)

Chen, J. J., Wang, T. M., Lan, C. F., Huang, Y. G., Chiang, I. T., Wu, S. K. et al., (2008). Healthy lifestyle -- Physical activity. In K. N. Kuo (Ed.), Healthy People 2020 (pp.183-224). Taipei: National Health Research Institutes. (in Chinese)

Cooper, C. L., Dewe, P., \& O'Driscoll, M. P. (2001). Organizational stress: A review and critique of theory, research, and applications. Thousand Oaks, Calif.: Sage.

Craig, C.L., Marshall, A.L., Sjostrom, M., Bauman, A. E., Booth, M. L., \& Ainsworth, B. E., et al. (2003). International physical activity questionnaire: 12-country reliability and validity. Medicine and Science in Sports and Exercise, 35(8), 13811395. 
Dodson, E. A., Lovegreen, S. L., Elliott, M. B., Haire-Joshu, D., \& Brownson, R. C. (2008). Worksite policies and environments supporting physical activity in midwestern communities. American Journal of Health Promotion, 23(1), 51-55.

Engbers, L. H., van Poppel, M. N., Chin, A. P. M. J., \& van Mechelen, W. (2005). Worksite health promotion programs with environmental changes: A systematic review. American Journal of Preventive Medicine, 29(1), 61-70.

Fan, K. T., \& Yen, L. L. (2008). Health promotion in the workplace: Comparison of the experiences of Taiwan and other countries. Taiwan Journal of Public Health, 27(4), 271-281. (in Chinese)

Foster, C., \& Hillsdon, M. (2004). Changing the environment to promote healthenhancing physical activity. Journal of Sports Sciences, 22(8), 755-769.

Hamilton, M. T., Hamilton, D. G., \& Zderic, T. W. (2007). Role of low energy expenditure and sitting in obesity, metabolic syndrome, type 2 diabetes, and cardiovascular disease. Diabetes, 56(11), 2655-2667.

Hamilton, M. T., Healy, G. N., Dunstan, D. W., Zderic, T. W., \& Owen, N. (2008). Too little exercise and too much sitting: Inactivity physiology and the need for new recommendations on sedentary behavior. Current Cardiovascular Risk Reports, 2(4), 292-298.

Hsu, J. H., \& Huang, S. L. (2001). Labour health promotion assistance plan for business health promotion (pp. I-1-I-31) (IOSH90-M341). Taipei: Institute of Occupational Safety and Health, Council of Labor Affairs. (in Chinese)

Huang, Y. C., Kao, Y. H., Chen, C. J., \& Hsu, J. H. (2003). The effects of exercise intervention on stages of exercise and physical activity for the workers in the worksite. Journal of Occupational Safety and Health, 11(1), 1-10. (in Chinese)

Jwo, J. C., Tong, S. Q., \& Yen, L. L. (1992). Experimental research on a worksite physical fitness promotion program. Physical Education Journal, 14, 193-206. (in Chinese)

Kahn, E. B., Ramsey, L. T., Brownson, R. C., Heath, G. W., Howze, E. H., Powell, K. E., et al. (2002). The effectiveness of interventions to increase physical activity. A systematic review. American Journal of Preventive Medicine, 22(4 Suppl), 73107.

Kao, Y. H., Lu, C. M., \& Huang, Y. C. (2002). Impact of a transtheoretical model on the psychosocial factors affecting exercise among workers. Journal of Nursing Research, 10(4), 303-310.

Karasek, R. (1979). Job demands, job decision latitude, and mental strain: Implications for job redesign. Administrative Science Quarterly, 24(2), 285-308. 
Karasek, R., Brisson, C., Kawakami, N., Houtman, I., Bongers, P., \& Amick, B. (1998). The Job Content Questionnaire (JCQ): An instrument for internationally comparative assessments of psychosocial job characteristics. Journal of Occupational Health Psychology, 3(4), 322-355.

Karasek, R., \& Theorell, T. (1990). Healthy work: Stress, productivity, and the reconstruction of working life. New York: Basic Books.

Katzmarzyk, P. T., Gledhill, N., \& Shephard, R. J. (2000). The economic burden of physical activity in Canada. Canadian Medical Association Journal, 163(11), 1435-1440.

Kremers, S. P., de Bruijn, G. J., Visscher, T. L., van Mechelen, W., de Vries, N. K., \& Brug, J. (2006). Environmental influences on energy balance-related behaviors: A dual-process view. International Journal of Behavioral Nutrition and Physical Activity, 3, 9.

Ku, P. W., Fox, K. R., McKenna, J., \& Peng, T. L. (2006). Prevalence of leisure-time physical activity in Taiwanese adults: Results of four national surveys, 2000-2004. Preventive Medicine, 43(6), 454-457.

Lee, Y. S., Huang, Y. C., \& Kao, Y. H. (2005). Physical activities and correlates of clinical nurses in Taipei municipal hospitals. Journal of Nursing Research, 13(4), 281-292.

Li, C. L., Tseng, H. M., Tseng, R. F., \& Lee, S. J. (2006). The effectiveness of an aerobic exercise intervention on worksite health-related physical fitness -- A case in a high-tech company. Chang Gung Medical Journal, 29(1), 100-106.

Lucove, J. C., Huston, S. L., \& Evenson, K. R. (2007). Workers' perceptions about worksite policies and environments and their association with leisure-time physical activity. American Journal of Health Promotion, 21(3), 196-200.

Marcus, B. H., Williams, D. M., Dubbert, P. M., Sallis, J. F., King, A. C., Yancey, A. K., et al. (2006). Physical activity intervention studies: What we know and what we need to know: A scientific statement from the American Heart Association Council on Nutrition, Physical Activity, and Metabolism (Subcommittee on Physical Activity); Council on Cardiovascular Disease in the Young; and the Interdisciplinary Working Group on Quality of Care and Outcomes Research. Circulation, 114(24), 2739-2752.

Matson-Koffman, D. M., Brownstein, J. N., Neiner, J. A., \& Greaney, M. L. (2005). A site-specific literature review of policy and environmental interventions that promote physical activity and nutrition for cardiovascular health: What works? American Journal of Health Promotion, 19(3), 167-193.

McLeroy, K. R., Bibeau, D., Steckler, A., \& Glanz, K. (1988). An ecological perspective on health promotion programs. Health Education Quarterly, 15(4), 351-377. 
Morris, J. N., Heady, J. A., Raffle, P. A., Roberts, C. G., \& Parks, J. W. (1953a). Coronary heart-disease and physical activity of work. Lancet, 265(6795), 1053 1057; contd.

Morris, J. N., Heady, J. A., Raffle, P. A., Roberts, C. G., \& Parks, J. W. (1953b).

Coronary heart-disease and physical activity of work. Lancet, 265(6796), 11111120; concl.

Plotnikoff, R. C., Prodaniuk, T. R., Fein, A. J., \& Milton, L. (2005). Development of an ecological assessment tool for a workplace physical activity program standard. Health Promotion Practice, 6(4), 453-463.

Pratt, M., Macera, C. A., \& Wang, G. (2000). Higher direct medical costs associated with physical inactivity. Physician and Sportsmedicine, 28(10), 63-70.

Prodaniuk, T. R., Plotnikoff, R. C., Spence, J. C., \& Wilson, P. M. (2004). The influence of self-efficacy and outcome expectations on the relationship between perceived environment and physical activity in the workplace. International Journal of Behavioral Nutrition and Physical Activity, 1(1), 7.

Pronk, N. P., Goodman, M. J., O’Connor, P. J., \& Martinson, B. C. (1999). Relationship between modifiable health risks and short-term health care charges. Journal of the American Medical Association, 282(23), 2235-2239.

Sallis, J. F., Bauman, A., \& Pratt, M. (1998). Environmental and policy interventions to promote physical activity. American Journal of Preventive Medicine, 15(4), 379397.

Sallis, J. F., \& Owen, N. (2002). Ecological models of health behavior. In K. Glanz, B. K. Rimer, F. M. Lewis (Eds.), Health behavior and health education: Theory, research, and practice (3rd ed., pp. 462-484). San Francisco: Jossey-Bass.

Spence, J. C., \& Lee, R. E. (2003). Toward a comprehensive model of physical activity. Psychology of Sport and Exercise, 4(1), 7-24.

Strecher, V. J., DeVellis, B. M., Becker, M. H., \& Rosenstock, I. M. (1986). The role of self-efficacy in achieving health behavior change. Health Education \& Behavior, 13(1), 73-91.

Taiwan 1111 Job Bank. (2007a). Physically inactive group in the workplace -- A survey of physical activity habits among office workers. Retrieved February 5, 2011, from http://project.1111.com.tw/zone/pr/headline.asp?autono=1702 (in Chinese)

Taiwan 1111 Job Bank. (2007b). A survey of voices from the information technology workers. Retrieved February 5, 2011, from http://www.1111.com.tw/zone/pr/headline.asp?autono=1677 (in Chinese) 
Taiwan Council of Labor Affairs, Executive Yuan. (2007). Career guide to industries: Computer system design services industry. Retrieved February 14, 2009, from $\mathrm{http} / / /$ statdb.cla.gov.tw/careerguide/ind/ind_detail.asp?section_id=1\&id_no=K07 2 (in Chinese)

Taiwan Science and Technology Advisory Group, Executive Yuan. (2007). Survey on supply and demand of manpower in the information technology service industry. Taipei: Author. (in Chinese)

Taylor, H. L., Klepetar, E., Keys, A., Parlin, W., Blackburn, H., \& Puchner, T. (1962). Death rates among physically active and sedentary employees of the railroad industry. American Journal of Public Health and the Nation's Health, 52, $1697-$ 1707.

Wang, F., McDonald T., Champagne, L. J., \& Edington, D. W. (2004). Relationship of body mass index and physical activity to health care costs among employees. Journal of Occupational and Environmental Medicine, 46(5), 428-436.

Wang, H. W., \& Chang, M. (2006). The effects of exercise intervention based on the transtheoretical model for employees in a worksite. Formosan Journal of Medicine, 10(3), 292-301. (in Chinese) 


\title{
CHAPTER 2
}

\section{Work Environment and Psychosocial Determinants of Physical Activity among White-Collar Workers: A Review of the Literature}

\begin{abstract}
Understanding how work environment and psychosocial determinants can influence worker physical activity is an occupational health research priority. Identifying the most promising determinants of physical activity is important for the development of effective interventions. METHODS: Research articles published in English (up to February 1, 2011) that described the relationship between work environment and psychosocial factors and physical activity among white-collar workers were reviewed. Each variable was evaluated across multiple studies for the direction of significant effects and the strength of support as a predictor of physical activity. RESULTS: Twenty-three studies were reviewed (4 prospective and 19 cross-sectional studies). Strong evidence was found for self-efficacy, perceived barriers or cons, and perceived benefit or pros as predictors of physical activity. Weak evidence was found for passive jobs, high strain jobs, job control, perceived workplace environments, intention, and social support. CONCLUSIONS: The findings suggest the need to examine these important work environment and psychosocial determinants of physical activity for different working populations in different settings, before they can be used to develop effective interventions.
\end{abstract}




\section{Introduction}

Physical activity is one of the leading health indicators that simultaneously represents a major public health concern (Taiwan Department of Health, 2010; U.S. Department of Health and Human Services [USDHHS], 2000). Regular physical activity has been found to be protective against numerous chronic diseases, such as cardiovascular disease, hypertension, diabetes, various cancers, obesity, osteoporosis, and mental illness (Andersen, Schnohr, Schroll, \& Hein, 2000; USDHHS, 2008; World Health Organization [WHO], 2002). However, the global prevalence of physical inactivity (doing very little or no physical activity) among adults was estimated to be $17 \%$ and caused almost 2 million deaths a year. The global prevalence of insufficient physical activity ( $<150$ minutes/week of moderate-intensity physical activity) averaged 41\% (WHO). In Taiwan, white-collar workers engaged in less physical activity (insufficient physical activity: $42 \%)$ when compared to other workers $(32 \%-39 \%)$ and the general population of adults aged 18 to 65 (38\%) (Chen et al., 2008). In a recent Internet survey of physical activity habits among 1,092 Taiwanese office workers, nearly $65 \%$ of the participants reported that they are physically inactive, with the information technology workers being the least active (Taiwan 1111 Job Bank, 2007).

Changes in today's living and working environments have led to inadequate physical activity and prolonged sitting, which are causally related to all-cause mortality (Andersen et al., 2000; Hamilton, Hamilton, \& Zderic, 2007; Patel et al., 2010; WHO, 2002). Sedentary work behaviors associated adverse health effects have been well documented (Morris, Heady, Raffle, Roberts, \& Parks, 1953a, 1953b; Taylor et al., 1962). As a result, the $\mathrm{WHO}$ emphasizes that physical inactivity is a growing threat to public 
health around the world, and that workplace health promotion interventions targeting physical activity and diet are an effective means to prevent chronic diseases and promote health (WHO, 2008).

Understanding determinants or mediating variables of physical activity is the foundation of the development of effective interventions (Baranowski, Cullen, Nicklas, Thompson, \& Baranowski, 2003; Brug, Oenema, \& Ferreira, 2005). Physical activity may be influenced by different personal (psychosocial) and environmental factors, which may differ across specific populations and under specific circumstances (Baranowski et al.; Brug et al.; Kremers et al., 2006; Spence \& Lee, 2003). Individual psychosocial factors have been widely investigated in studies of worker physical activity. Only more recent studies have focused on identifying either organizational (e.g., worksite environments and policies) or individual (e.g., job strain and its components—-job demands and job control) level factors in the work environment associated with physical activity. Literature reviews have been conducted on the effectiveness of worksite physical activity interventions (Dishman, Oldenburg, O’Neal, \& Shephard, 1998; Pronk, 2009; Proper et al., 2003), worksite health promotion programs with policy and/or environmental changes to promote physical activity (Engbers, van Poppel, Chin, \& van Mechelen, 2005; Matson-Koffman, Brownstein, Neiner, \& Greaney, 2005), and determinants of employee participation in physical activity (Kaewthummanukul \& Brown, 2006), but a review of work environment and psychosocial determinants of physical activity is lacking. This paper updates and extends the earlier review by Kaewthummanukul and Brown, focusing on work environment and psychosocial determinants of physical activity behavior among white-collar workers. 


\section{Methods}

Search strategy. The following sources were searched to identify potential research articles: online databases (e.g., PubMed, PsycINFO, and CINAHL), reference lists of included papers, and hand search of journals. Articles were retrieved by using a combination of the search terms: "physical activity or exercise," and "workers or employees," and "psychosocial or work environment factors or determinants or predictors or job strain."

Inclusion criteria. Only papers that were published in English and that described the relationship between psychosocial or work environment factors (independent variables) and physical activity or exercise behavior (dependent variable) in (predominantly) white-collar workers were considered for review. No time restriction was placed on publication date (up to February 1, 2011).

\section{Results}

Twenty-three articles were identified as meeting the criteria, 13 of which were categorized under the work environment determinants and 10 of which were categorized under the psychosocial determinants. Nineteen studies used cross-sectional design or data, including papers that presented only baseline results of a prospective or quasiexperimental design. Four studies were prospective (ranging from 8 weeks to 5 years). Sixteen of the 23 studies used an established theoretical framework to guide their research endeavors, including Karasek's (1979) demand/control model (also termed job strain model), Theory of Planned Behavior (TPB), transtheoretical model (TTM), and Social Cognitive Theory (SCT). All studies reviewed relied on self-report measures of all variables. Nine studies examined associations of work environment and/or psychosocial 
determinants with leisure-time physical activity (LTPA). Seven studies examined associations with general physical activity (including light, moderate, and vigorous intensity physical activity, as well as physical activity of daily living). Six studies examined associations with exercise (a subset of physical activity referring to relatively structured activities, such as purposeful walking and jogging). Two studies examined associations with work-break or workplace physical activity. Tables 2.1 and 2.2 summarize the samples, designs, theoretical frameworks used, work environment and psychosocial factors measured, type of physical activity outcome, and major findings and their direction. Tables 2.3 and 2.4 highlight the direction of associations (significant at $p$ $<.05$ and nonsignificant) between job strain variables and physical activity, and between psychosocial variables and physical activity across studies, respectively.

\section{Studies Examining the Effects of Work Environment on Physical Activity}

Organizational work environment factors. Prodaniuk, Plotnikoff, Spence, and Wilson (2004) used the six-item Perceived Workplace Environment Scale (PWES) to measure the perceptions of workplace environmental support for physical activity. Each PWES item assesses perceptions about one of the six environmental dimensions of the ecological workplace physical activity model. The results showed that each PWES item or global PWES construct was weakly but significantly and positively related to LTPA and workplace physical activity. Moreover, the relationship between perceived workplace environment and workplace physical activity was partially mediated by self-efficacy in the cross-sectional (Prodaniuk et al.) and longitudinal (Plotnikoff, Pickering, Flaman, \& Spence, 2010) analyses in the same cohort. The results yielded support for self-efficacy as a mediator across three time points (at baseline, 6 months, and 12 months). 
Two studies examined the relationship of worksite policies and environments with physical activity (Dodson, Lovegreen, Elliott, Haire-Joshu, \& Brownson, 2008; Lucove, Huston, \& Evenson, 2007). Lucove et al. found that subsidies for health club use were positively related to both LTPA and work-break physical activity. However, on-site exercise facility was only positively related to LTPA, whereas paid time for non-workrelated physical activity and a safe place to walk outside work were only positively related to work-break physical activity. Dodson et al. found that workers with accessible exercise facilities and equipment, stairways, and personal services (e.g., fitness testing and counseling) at worksites were more likely to meet physical activity recommendations than those without these environments and services. Further, the number of policies or environments available and visible in the workplace was associated with the likelihood of meeting physical activity recommendations. Other variables not found to be correlated with physical activity included group services (e.g., exercise classes and health fairs), sponsored sports teams, resource materials (e.g., brochures, posters, and videos), reduced health insurance premiums, other monetary incentives, and flexible work schedule. Additionally, in one study of employed women, Tavares, Plotnikoff, \& Loucaides (2009) found no relationship between perceived physical environment and physical activity, and they used a single item to measure the degree of access to a place where participants can be physically active.

Unfortunately, $69 \%$ of 977 U.S. workers reported no policies or environments that support physical activity in their workplace (Dodson et al., 2008). The most commonly reported policies or environments were accessible stairways (57\%) and a safe place to walk outside work (56\%), whereas paid time for non-work-related physical activity 
(15\%), facilities (e.g., gym, locker, shower) (15\%), and equipment (e.g., treadmill, cycle, weights) (11\%) were the least commonly reported (Dodson et al.; Lucove et al., 2007). In contrast, a study (not included in this review) investigating barriers and incentives for worksite health promotion services and policies indicated that the most favored physical activity services cited by 2,337 U.S. workers were fitness centers $(81 \%)$ and on-site exercise classes (55\%), and the most supported policy was giving paid time to exercise at work (89\%) (Kruger, Yore, Bauer, \& Kohl, 2007). Noticeably, there was a discrepancy between workers' desires or expectations and the organization's ability to meet them.

Individual work environment factors. In recent years, much attention has been given to the effects of working conditions on health behaviors, including physical activity or sedentary behavior. Karasek's (1979) demand/control (job strain) model appears to be the most widely used theory, which describes two dimensions of the psychosocial job characteristics: psychological job demands and job control. Job demands are the psychological demands of work with regard to workload, work pace, organizational constraints on completing work, and conflicting demands. Job control refers to job decision latitude, and it is defined based on measures of skill discretion as well as decision authority (Karasek et al., 1998). According to the model, low control combined with high demands represents a "high strain job," which may lead to psychological strain and even physiological health outcomes, whereas high control combined with low demands represents a "low strain job," which leads to relatively less strain. High control combined with high demands represents an "active job," indicating good strain, while low control combined with low demands represents a "passive job," which may lead to negative job learning (Karasek et al., 1998). 
Table 2.3 presents both significant and nonsignificant associations between job strain variables and physical activity, stratified by gender. The relationships between job demands, job control, job strain (ratio of demands to control), and physical activity were somewhat inconsistent and varied between sexes. For example, women with active jobs were more likely to be physically active (Hellerstedt \& Jeffery, 1997), while men and older workers with active jobs were less likely to be active (Kouvonen et al., 2005). Women with low strain jobs were less likely to be physically active, while men with low strain jobs were more likely to be active (Hellerstedt \& Jeffery). Nevertheless, passive jobs, high strain jobs, and low job control had a relatively consistent relationship with lower levels of physical activity or exercise in both men and women (Brisson, Larocque, Moisan, Vezina, \& Dagenais, 2000; Gimeno et al., 2009; Hellerstedt \& Jeffery; Kouvonen et al.; Lallukka et al., 2008).

However, other studies did not find an association between exercise or sedentary behavior and job demands, job control, or job strain (Kaewthummanukul, Brown, Weaver, \& Thomas, 2006; Landsbergis et al., 1998; Payne, Jones, \& Harris, 2002, 2005). Two studies (Payne et al., 2002, 2005) were conducted in a computer company in the U.K., which did not stratify analyses by gender and they found no relationship between exercise and job demands or job control. Nevertheless, Payne et al. (2002) indicated that high strain workers reported significantly lower exercise self-efficacy, lower perceived behavioral control, and less exercise than low strain workers. In addition, intenders who failed to exercise reported higher job demands than intenders who succeeded in exercising. A one-unit increase in the job demands score was associated with a 7\% increase of failing to implement exercise intention $(\mathrm{OR}=.93)$ (Payne et al.). 
A comparative study (Lallukka et al., 2008) that used three cohorts of white-collar workers from Britain, Finland, and Japan concluded that job strain had weak and inconsistent association with physical inactivity across gender and cohorts. For instance, passive jobs and high strain jobs were found to be associated with a greater likelihood of physical inactivity for British men and Finnish women only. None of the job strain variables were associated with physical inactivity for Japanese men and women, British women, and Finnish men.

\section{Studies Examining the Effects of Psychosocial Factors on Physical Activity}

Studies examined several constructs that can broadly be classified as outcome expectations (used interchangeably with outcome expectancies), defined in the SCT as beliefs about the expected benefits and costs for performing a behavior in particular situations (Bandura, 1986, 2004; Baranowski, Perry, \& Parcel, 2002). In a review of the role of outcome expectancy in physical activity behavior, Williams, Anderson, and Winett (2005) demonstrated that outcome expectancy is labeled and conceptualized in a variety of ways within different theories, but all include expected outcomes of a behavior. The authors noted that perceived benefits are the same as positive outcome expectancies. Perceived barriers are not the same as negative outcome expectancies; however, they share similar beliefs since barriers are based partly on expected negative outcomes. An example is that the perception of lack of time as a barrier is based on the expectation that physical activity will take away from the time someone has for his/her work, family or friends. Table 2.4 shows anticipated affective reaction, attitude, barriers, cons, benefits, pros, enjoyment, fear, response efficacy, severity, and vulnerability under the label of outcome expectations. 
Perceived benefits or pros of physical activity. Six of nine studies

(Kaewthummanukul et al., 2006; Nishida, Suzuki, Wang, \& Kira, 2004; Prodaniuk et al., 2004; Purath, 2006; Steinhardt \& Dishman, 1989; Tavares et al., 2009) reported generally positive relationships between perceived benefits or pros of engaging in physical activity and physical activity. However, there are some differences in studies according to analysis based on the factor or item level and different working groups (women vs. men or women with young children vs. women without young children). At the factor level, all two of the studies (Nishida et al.; Steinhardt \& Dishman) revealed that psychological benefits were positively associated with stages of physical activity or participation in the onsite fitness program, whereas the results of social and body image/health benefits were found to be inconsistent in these two studies. Nishida et al. reported that social benefits were positively associated with stages of physical activity among Japanese male workers, but Steinhardt and Dishman found that U.S. fitness program adherents were less likely than nonadherents to rate social benefits as expected outcomes of physical activity. In contrast, U.S. fitness program adherents were more likely than nonadherents to value body image/health outcomes (Steinhardt \& Dishman), and neither health nor weight control benefits were associated with stages of physical activity in Japanese male workers (Nishida et al.). At the item level, physically active women were more likely than inactive women to view that physical activity contributes to decreased stress and an increased positive outlook on life (Purath). Additionally, women without young children showed an association between pros of physical activity and stages of physical activity, while women with young children did not (Tavares et al.). 
Perceived barriers or cons of physical activity. Nine of ten studies (Chen \& Chang, 2004; Kaewthummanukul et al., 2006; Kao, Lu, \& Huang, 2002; Nishida et al., 2004; Prodaniuk et al., 2004; Purath, 2006; Sassen, Kok, Schaalma, Kiers, \& Vanhees, 2010; Steinhardt \& Dishman, 1989; Tavares et al., 2009) reported generally negative relationships between perceived barriers or cons of engaging in physical activity and physical activity, although some differences were observed in studies. Two studies (Nishida et al.; Steinhardt \& Dishman) consistently showed that barriers regarding physical or obstacles (both factors include elements of too boring and too inconvenient) were negatively associated with stages of physical activity or participation in the onsite fitness program. However, time barriers were found to be a predictor of physical activity in U.S. workers (Steinhardt \& Dishman), but not in Japanese male workers (Nishida et al.). Physically active women were less likely than inactive women to cite bad weather, time, and tired as barriers to physical activity (Purath). Women with young children, compared to women without young children, had a relatively strong association between cons of physical activity (e.g., perceived less time for family and friends if engaging in physical activity; too tired to engage in physical activity because of other daily responsibilities) and stages of physical activity (Tavares et al.).

Self-efficacy for physical activity. Ten of eleven studies (Chen \& Chang, 2004; Kaewthummanukul et al., 2006; Kao et al., 2002; Lee, Huang, \& Kao, 2005; Nishida et al., 2004; Payne et al., 2002; Prodaniuk et al., 2004; Purath, 2006; Sassen et al., 2010; Tavares et al., 2009) reported a positive relationship between self-efficacy for physical activity and physical activity. The eleventh study found no relationship between these two variables, likely due to the low reliability of the self-efficacy measure (Cronbach's 
alpha $=.60)$ (Jackson, Smith, \& Conner, 2003). Four studies (Jackson et al.; Payne et al., 2002, 2005; Sassen et al.) assessed perceived control or perceived behavioral control (constructs similar to SCT's concept of self-efficacy) and found no association with exercise or LTPA.

Social support. Each of the three studies (Chen \& Chang, 2004;

Kaewthummanukul et al., 2006; Tavares et al., 2009) reported generally positive relationships between social support and physical activity. In addition, Tavares et al. pointed out that women without young children perceived a stronger association between social support and stages of physical activity, as compared to women with young children.

Intention. Three of five studies (Payne et al., 2002; Sassen et al., 2010; Tavares et al., 2009) that examined the relationship between intention for future engagement in physical activity and physical activity behavior found a strong, positive association between the two variables.

Other variables. As presented in Table 2.4, relationships between several additional psychosocial variables and physical activity were evaluated. Descriptive norms (sample item: "Are those people who are important to you physically active?") was found to be positively associated with physical activity in one of the two studies (Sassen et al., 2010). Motivation was shown to be positively related to physical activity in one study (Kaewthummanukul et al., 2006). Higher levels of self-identity (sample item: "I see myself as a physically active person") were associated with higher levels of LTPA in one study (Jackson et al., 2003). Finally, of the three studies (Jackson et al.; Sassen et al.; Tavares et al., 2009) that examined the relationship between subjective norms or injunctive norms and physical activity, neither of which was found to be significant. 


\section{Discussion}

Results from the reviewed studies showed diversity in sample characteristics, research design, measures, and employing psychosocial constructs derived from a variety of theories, such as job strain (demand/control) model, TPB, TTM, and SCT. Each study and variable was individually rated based on the direction of significant effects and the strength of support as a predictor of physical activity. The pattern of findings was summarized in Tables 2.3 and 2.4.

Overall, positive, albeit weak, associations between perceived workplace environments or policies and physical activity (e.g., workplace and work-break physical activity or LTPA) were identified in three studies. Job strain variables had some independent, but relatively small, effects on LTPA or exercise in six studies, with passive jobs, high strain jobs, and job control demonstrating more evidence. As for the psychosocial variables, self-efficacy, barriers or cons, and benefit or pros were the most commonly assessed constructs and provided more evidence for associations with physical activity, with self-efficacy emerging as the strongest predictor. Intention and social support were also observed to have some evidence for predicting physical activity. Finally, a number of other constructs were observed to have insufficient evidence for predicting physical activity among white-collar workers, such as job demands, motivation, enjoyment, fear, self-identify, and descriptive norms. These merit further study.

Much of the research on worker physical activity to date has focused more on individual psychosocial and job strain variables, but researchers are beginning to explore the potential influence of worksite environments and policies on physical activity. The paucity of research examining the effects of workplace environments on physical activity 
is most likely due to the challenge of operationalizing key constructs and the lack of theoretically-based and psychometrically sound measures of workplace physical activity environments. Only one reliable and valid measure of perceived workplace environment (Prodaniuk et al., 2004) was identified in this review. On the contrary, theories and measures of psychosocial constructs are more well-developed. Therefore, it would be premature to suggest that psychosocial factors are more influential than environmental factors. Alternatively, more efforts are needed to establish a consensus on the conceptual and operational definitions and theoretical frameworks to foster the empirical examination of the concept of workplace physical activity environments.

Very few of the reviewed studies examined the combined effects of work environment and psychosocial variables on worker physical activity, and even fewer included a mediator variable. The findings of this review support the recommendation of an earlier review of physical activity intervention studies, indicating that there is a pressing need to examine how environmental and psychosocial variables interact in their association with physical activity (Marcus et al., 2006). Moreover, to fully understand how work environment may affect worker physical activity, examining both perspectives (e.g., workplace environments and job strain) simultaneously will contribute to our understanding of it. Furthermore, there have been recent calls to examine the potential mediation effects of psychosocial factors on the relationship of environmental factors and physical activity, in an attempt to guide development of interventions (Baranowski et al., 2003; Brug et al., 2005; Kremers et al., 2006; Prodaniuk et al., 2004; Sallis \& Owen, 2002; Spence \& Lee, 2003). 
The findings of the present review exhibit diversity in the population groups (e.g., Asian, North American, and European), research settings (e.g., public and private sector worksites, hospitals, and communities), and measures (varying in the number of items, reliability and validity) across studies. Although the target population in this review is predominantly white-collar workers, variations in job characteristics and work environments are expected within and across studies. This can affect workers' levels of physical activity at work. For example, nurses' job tasks are very different from that of other workers studied. Lee et al. (2005) reported that hospital nurses are significantly more physically active than office workers during the working day, whereas on a nonworking day, office workers are likely more active than nurses. These differences may account for some inconsistent findings in some of the studies reviewed here. Also, generalizations across studies are difficult to make due to diverse measurement instruments used. There is a clear need for standardized and validated measures to be used across studies, in order to determine the theoretically consistent patterns of findings.

The majority of studies were descriptive or exploratory $(n=19)$. The use of a cross-sectional design can help identify the most salient constructs for further study, but questions of causality cannot be demonstrated. Of the few findings from prospective studies, only one study employed mediator analyses, which allowed the researchers to disentangle the causal direction and meaning of the relationship between the predictor variable and the outcome variable. The examination of potential mediators in intervention studies is crucial because it offers a means of understanding how or why an intervention works (Bennett, 2000). 
In conclusion, the primary purpose of this literature review was to determine the most promising work environment and psychosocial determinants of physical activity among white-collar workers. Strong evidence was found for psychosocial variables, including self-efficacy, barriers or cons, and benefit or pros. Weak evidence was found for work environment variables (e.g., passive jobs, high strain jobs, job control, and perceived workplace environments) as well as such variables as intention and social support. The findings suggest the need to examine these important work environment and psychosocial determinants of physical activity for different working populations in different settings, before they can be used to develop effective interventions. In doing so, it is recommended that prospective designs with validated measures and mediation analyses be used to verify the strength of these potential predictors of physical activity. 
Table 2.1 Characteristics and Main Findings of Studies Examining Relationships of Work Environment Determinants with Physical Activity among White-Collar Workers

\begin{tabular}{|c|c|c|c|c|c|c|c|}
\hline Study & Sample & Design & $\begin{array}{l}\text { Theoretical } \\
\text { framework }\end{array}$ & $\begin{array}{c}\text { Determinant/ } \\
\text { Predictor }\end{array}$ & $\begin{array}{l}\text { Association with } \\
\text { PA/Exercise }\end{array}$ & $\begin{array}{c}\text { PA/Exercise } \\
\text { measurement }\end{array}$ & Notes \\
\hline $\begin{array}{l}\text { Brisson et } \\
\text { al. }(2000)\end{array}$ & $\begin{array}{l}\mathrm{N}=6995(50 \% \text { men }) \\
\text { Age=18-65 yrs } \\
\mathrm{M}=41 \text { yrs (men) } \\
\mathrm{M}=39 \text { yrs (women) } \\
\text { Setting: } 20 \text { public } \\
\text { and semi-public } \\
\text { organizations and } 1 \\
\text { private insurance } \\
\text { company in Canada }\end{array}$ & $\begin{array}{l}\text { Cross- } \\
\text { sectional }\end{array}$ & $\begin{array}{l}\text { Job strain } \\
\text { model }\end{array}$ & $\begin{array}{l}\text { Job demands } \\
\text { (1) low } \\
\text { (2) } \\
\text { (3) } \\
\text { (4) high } \\
\text { Job control } \\
\text { (1) low } \\
\text { (2) } \\
\text { (3) } \\
\text { (4) high } \\
\text { Job strain } \\
\text { - Low strain job } \\
\text { - Active job } \\
\text { - Passive job } \\
\text { - High strain job }\end{array}$ & $\begin{array}{l}\text { reference group } \\
\text { ns/ns F/M } \\
\mathrm{OR}=1.3^{*} / \mathrm{ns} \mathrm{F} / \mathrm{M} \\
\mathrm{ns} / \mathrm{ns} \mathrm{F} / \mathrm{M} \\
\text { ns/OR=1.3* F/M } \\
\mathrm{ns} / \mathrm{ns} \mathrm{F} / \mathrm{M} \\
\mathrm{ns} / \mathrm{ns} \mathrm{F} / \mathrm{M} \\
\text { reference group } \\
\text { reference group } \\
\mathrm{ns} / \mathrm{ns} \mathrm{F} / \mathrm{M} \\
\mathrm{ns} / \mathrm{OR}=1.3^{*} \mathrm{~F} / \mathrm{M} \\
\mathrm{ns} / \mathrm{OR}=1.2^{*} \mathrm{~F} / \mathrm{M}\end{array}$ & $\begin{array}{l}\text { - } 1 \text { exercise session: } \\
\text { a rigorous PA during } \\
\text { leisure time that } \\
\text { lasted } \geq 20 \text { minutes } \\
\text { - Sedentary } \\
\text { behavior: exercise } \\
<1 \text { time/wk over the } \\
\text { last } 6 \mathrm{mos}\end{array}$ & $\begin{array}{l}\text { Sedentary behavior } \\
\text { were higher in } \\
\text { women }(47 \%) \text { than } \\
\text { in men }(37 \%)\end{array}$ \\
\hline $\begin{array}{l}\text { Dodson et } \\
\text { al. }(2008)\end{array}$ & $\begin{array}{l}\mathrm{N}=977 \text { (mostly } \\
\text { sitting or standing } \\
\text { while at work; } 25 \% \\
\text { men) } \\
\text { Median age=43 yrs } \\
\text { Setting: midwestern } \\
\text { communities in the } \\
\text { US }\end{array}$ & $\begin{array}{l}\text { Cross- } \\
\text { sectional }\end{array}$ & None & $\begin{array}{l}\text { Structured: } \\
\text { Facilities for PA } \\
\text { Equipment } \\
\text { Subsidized } \\
\text { memberships } \\
\text { Group services } \\
\text { Sponsored sports } \\
\text { teams } \\
\text { Nonstructured: } \\
\text { Accessible } \\
\text { stairways } \\
\text { Personal services } \\
\text { Time for PA }\end{array}$ & $\begin{array}{l}\mathrm{OR}=1.7(1.1-2.8) \\
\mathrm{OR}=2.0(1.2-3.5) \\
\mathrm{ns} \\
\mathrm{ns} \\
\mathrm{ns}\end{array}$ & $\begin{array}{l}\text { PA items derived } \\
\text { from the Behavioral } \\
\text { Risk Factor } \\
\text { Surveillance System } \\
\text { Survey }\end{array}$ & $\begin{array}{l}\text { - } 69 \% \text { reported no } \\
\text { PA policies or } \\
\text { environments } \\
\text { available at } \\
\text { worksites } \\
\text { - } 38 \% \text { met PA } \\
\text { recommendation } \\
\text { via moderate or } \\
\text { vigorous PA, and } \\
21 \% \text { only via } \\
\text { walking }\end{array}$ \\
\hline
\end{tabular}




\begin{tabular}{|c|c|c|c|c|c|c|c|}
\hline & & & & $\begin{array}{l}\text { Resource } \\
\text { materials } \\
\text { Reduced health } \\
\text { insurance } \\
\text { Other monetary } \\
\text { incentives }\end{array}$ & $\begin{array}{l}\text { ns } \\
\text { ns } \\
\text { ns }\end{array}$ & & \\
\hline $\begin{array}{l}\text { Gimeno et } \\
\text { al. (2009) }\end{array}$ & $\begin{array}{l}\mathrm{N}=6085 \text { office staff } \\
(71 \% \text { men }) \\
\text { Age }=35-55 \text { yrs } \\
\text { Setting: } 20 \text { civil } \\
\text { service departments } \\
\text { in the UK }\end{array}$ & $\begin{array}{l}\text { Prospecti } \\
\text { ve }\end{array}$ & None & $\begin{array}{l}\text { Passive jobs } \\
\text { (cumulated over } 3 \\
\text { phases) } \\
\text { - None } \\
\text { - } 1 \text { phase } \\
\text { - } 2 \text { phases } \\
\text { - } 3 \text { phases }\end{array}$ & $\begin{array}{l}\text { (Results for men) } \\
\text { reference } \\
\mathrm{ns} \\
\mathrm{PR}=1.1(1.0-1.3) \\
\mathrm{PR}=1.3(1.2-1.5)\end{array}$ & $\begin{array}{l}\text { LTPA (2 items): } \\
\text { Average number of } \\
\text { hours per week spent } \\
\text { in "moderately } \\
\text { energetic" and } \\
\text { "vigorous" PA }\end{array}$ & $\begin{array}{l}\text { - Low levels of } \\
\text { LTPA: }<1.5 \mathrm{hrs} / \mathrm{wk} \\
\text { of at least } \\
\text { moderately } \\
\text { energetic LTPA } \\
\text { - Cumulative effect } \\
\text { of passive jobs on } \\
\text { low levels of } \\
\text { LTPA applied only } \\
\text { to men }\end{array}$ \\
\hline $\begin{array}{l}\text { Hellerstedt } \\
\text { \& Jeffery } \\
(1997)\end{array}$ & $\begin{array}{l}\mathrm{N}=3843(49 \% \text { men) } \\
\mathrm{M}=39 \text { yrs (men) } \\
\mathrm{M}=38 \text { yrs (women) } \\
\text { Setting: } 12 \text { public } \\
\text { and } 20 \text { private } \\
\text { worksites in } \\
\text { Minnesota, USA }\end{array}$ & $\begin{array}{l}\text { Cross- } \\
\text { sectional }\end{array}$ & $\begin{array}{l}\text { Job strain } \\
\text { model }\end{array}$ & $\begin{array}{l}\text { Job demands } \\
\text { (1) low } \\
\text { (2) } \\
\text { (3) } \\
\text { (4) high } \\
\text { Job control } \\
\text { (1) low } \\
\text { (2) } \\
\text { (3) } \\
\text { (4) high } \\
\text { Job strain } \\
\text { - High strain job } \\
\text { - Low strain job } \\
\text { - Active job } \\
\text { - Passive job }\end{array}$ & 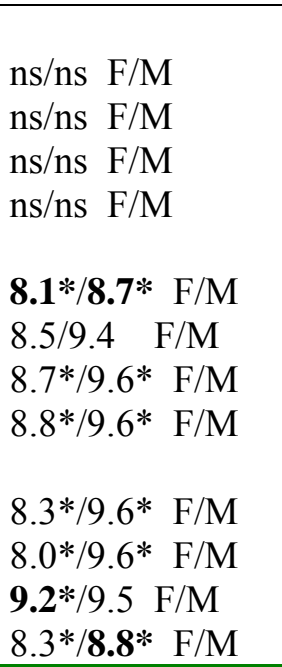 & $\begin{array}{l}\text { Exercise sessions: } \\
\text { sum the responses to } \\
12 \text { items about the } \\
\text { weekly frequency of } \\
\text { specific PA }\end{array}$ & \\
\hline
\end{tabular}




\begin{tabular}{|c|c|c|c|c|c|c|c|}
\hline $\begin{array}{l}\text { Kouvonen } \\
\text { et al. } \\
(2005)\end{array}$ & $\begin{array}{l}\mathrm{N}=46573(19 \% \\
\text { men) } \\
\text { Age: } 17-64 \text { yrs } \\
\text { Setting: Finnish } \\
\text { public sector in } 10 \\
\text { municipalities and } \\
21 \text { hospitals }\end{array}$ & $\begin{array}{l}\text { Cross- } \\
\text { sectional }\end{array}$ & $\begin{array}{l}\text { Demand/co } \\
\text { ntrol model } \\
\text { of job strain }\end{array}$ & $\begin{array}{l}\text { Job strain } \\
\text { - Low strain job } \\
\text { - Active job } \\
\text { - Passive job } \\
\text { - High strain job } \\
\text { Job control } \\
\text { - high } \\
\text { - intermediate } \\
\text { - low } \\
\text { Job demands } \\
\text { - low } \\
\text { - intermediate } \\
\text { - high }\end{array}$ & $\begin{array}{l}\text { reference group } \\
\mathrm{ns} / * * \mathrm{~F} / \mathrm{M} \\
* * * / * * * \mathrm{~F} / \mathrm{M} \\
* * * / * * * \mathrm{~F} / \mathrm{M} \\
\text { reference group } \\
* * * / \mathrm{ns} \mathrm{F} / \mathrm{M} \\
* * * / * * * \mathrm{~F} / \mathrm{M} \\
\text { reference group } \\
\mathrm{ns} / \mathrm{ns} \mathrm{F} / \mathrm{M} \\
\mathrm{ns} / \mathrm{ns} \mathrm{F} / \mathrm{M}\end{array}$ & $\begin{array}{l}\text { LTPA: average } \\
\text { amount of time spent } \\
\text { per week on leisure } \\
\text { and on the journey to } \\
\text { and from work in PA } \\
\text { corresponding to the } \\
\text { activity intensity of } \\
\text { walking, vigorous } \\
\text { walking, jogging, } \\
\text { and running (MET } \times \\
\text { h/wk) }\end{array}$ & $\begin{array}{l}\text { - Men engaged in } \\
\text { more LTPA than } \\
\text { women ( } 36 \text { vs. } 31 \\
\text { MET×h/wk) } \\
\text { - Active jobs were } \\
\text { related to low } \\
\text { LTPA only in men } \\
\text { and older workers } \\
\text { - High job } \\
\text { demands were } \\
\text { related to low } \\
\text { LTPA only in } \\
\text { upper nonmanual } \\
\text { workers }\end{array}$ \\
\hline $\begin{array}{l}\text { Lallukka et } \\
\text { al. (2004) }\end{array}$ & $\begin{array}{l}\mathrm{N}=6243 \text { employees } \\
(20 \% \text { men }) \\
\text { Age=40-60 yrs } \\
\text { Setting: Helsinki, } \\
\text { Finland }\end{array}$ & $\begin{array}{l}\text { Cross- } \\
\text { sectional }\end{array}$ & None & $\begin{array}{l}\text { Job strain } \\
\text { - High strain job } \\
\text { - Passive job } \\
\text { - Low strain job } \\
\text { - Active job } \\
\text { Work fatigue } \\
\text { Work overtime } \\
\text { Physically } \\
\text { strenuous work } \\
\text { Mentally } \\
\text { strenuous work } \\
\text { Work-home } \\
\text { interface }\end{array}$ & $\begin{array}{l}\text { (for women only) } \\
\text { reference group } \\
\mathrm{ns} \\
\mathrm{ns} \\
\mathrm{ns} \\
\mathrm{OR}=0.8(0.6-0.9) \\
\mathrm{ns} \\
\mathrm{OR}=1.2(1.0-1.4) \\
\mathrm{ns} \\
\mathrm{OR}=1.3(1.1-1.4)\end{array}$ & $\begin{array}{l}\text { - LTPA over the past } \\
12 \text { mos } \\
\text { - Recommended PA } \\
\text { level (>30 MET } \times \\
\mathrm{h} / \text { wk) was a } \\
\text { dependent variable } \\
\text { in the logistic } \\
\text { regression analyses }\end{array}$ & $\begin{array}{l}\text { - In men, none of } \\
\text { the work } \\
\text { conditions were } \\
\text { related to PA in the } \\
\text { final model } \\
\text { - } 33 \% \text { of women } \\
\text { and } 34 \% \text { of men } \\
\text { met recommended } \\
\text { PA level }\end{array}$ \\
\hline $\begin{array}{l}\text { Lallukka et } \\
\text { al. }(2008)\end{array}$ & $\begin{array}{l}\mathrm{n}_{1}=3397(72 \% \text { men; } \\
\text { Britain) } \\
\mathrm{n}_{2}=6070(20 \% \text { men; }\end{array}$ & $\begin{array}{l}\text { Cross- } \\
\text { sectional } \\
\text { comparat }\end{array}$ & $\begin{array}{l}\text { Job strain } \\
\text { model }\end{array}$ & $\begin{array}{l}\text { Britain } \\
\text { Job strain } \\
\text { - Low strain job }\end{array}$ & $\begin{array}{l}\text { (for men only) } \\
\text { reference group }\end{array}$ & $\begin{array}{l}\text { - LTPA: amount and } \\
\text { intensity (mild, } \\
\text { moderate, and }\end{array}$ & $\begin{array}{l}\text { - None of the job } \\
\text { strain variables } \\
\text { were related to }\end{array}$ \\
\hline
\end{tabular}




\begin{tabular}{|c|c|c|c|c|c|c|c|}
\hline & $\begin{array}{l}\text { Finland) } \\
\mathrm{n}_{3}=2213 \text { ( } 71 \% \text { men; } \\
\text { Japan) } \\
\text { Age=45-60 yrs } \\
\text { Settings: } 20 \text { civil } \\
\text { service departments } \\
\text { in London; city of } \\
\text { Helsinki; a local } \\
\text { government in } \\
\text { Japan }\end{array}$ & ive & & $\begin{array}{l}\text { - Active job } \\
\text { - Passive job } \\
\text { - High strain job } \\
\text { Work overtime } \\
\text { Finland } \\
\text { Job strain } \\
\text { - Low strain job } \\
\text { - Active job } \\
\text { - Passive job } \\
\text { - High strain job } \\
\text { Work overtime }\end{array}$ & $\begin{array}{l}\mathrm{ns} \\
\mathrm{OR}=1.6(1.2-2.3) \\
\mathrm{OR}=1.9(1.3-2.7) \\
\mathrm{ns} \\
\text { (for women only) } \\
\text { reference group } \\
\mathrm{ns} \\
\mathrm{OR}=1.3(1.1-1.6) \\
\mathrm{OR}=1.3(1.0-1.6) \\
\mathrm{ns}\end{array}$ & $\begin{array}{l}\text { vigorous) } \\
\text { - Physical inactivity: } \\
\leq 1 \text { h of LTPA/wk or } \\
\text { the lowest quintile of } \\
\text { the distribution of } \\
\text { the sum as a cutoff } \\
\text { point }\end{array}$ & $\begin{array}{l}\text { physical inactivity } \\
\text { in Japanese men } \\
\text { and women, } \\
\text { British women, } \\
\text { and Finnish men } \\
\text { - } 18-23 \% \text { were } \\
\text { physically inactive }\end{array}$ \\
\hline $\begin{array}{l}\text { Landsbergi } \\
\text { s et al. } \\
(1998)\end{array}$ & $\begin{array}{l}\mathrm{N}=202 \text { men } \\
\text { Age=30-60 yrs } \\
\text { Setting: } 9 \text { public } \\
\text { and private sector } \\
\text { worksites in New } \\
\text { York, USA }\end{array}$ & $\begin{array}{l}\text { Cross- } \\
\text { sectional }\end{array}$ & $\begin{array}{l}\text { Job strain } \\
\text { model }\end{array}$ & $\begin{array}{l}\text { Job control } \\
\text { Job demands } \\
\text { High job strain }\end{array}$ & $\begin{array}{l}\mathrm{ns} \\
\mathrm{ns} \\
\mathrm{ns}\end{array}$ & $\begin{array}{l}\text { Sedentary behavior } \\
\text { assessed by } 3 \text { items: } \\
\text { - Are you exercising } \\
\text { regularly at the } \\
\text { present time? } \\
\text { - If yes, how many } \\
\text { times per week? } \\
\text { - When you exercise, } \\
\text { what is the average } \\
\text { length of time? } \\
\text { - Sedentary: exercise } \\
<2 \text { h/wk }\end{array}$ & \\
\hline $\begin{array}{l}\text { Lucove et } \\
\text { al. (2007) }\end{array}$ & $\begin{array}{l}\mathrm{N}=987 \text { employees } \\
\text { Age } \geq 18 \text { yrs } \\
\text { Setting: community } \\
\text { in } 6 \text { North Carolina } \\
\text { counties in the US }\end{array}$ & $\begin{array}{l}\text { Cross- } \\
\text { sectional }\end{array}$ & None & $\begin{array}{l}\text { Subsidy for health } \\
\text { club } \\
\text { Paid time for non- } \\
\text { work-related PA } \\
\text { On-site fitness } \\
\text { facility } \\
\text { Safe place to walk }\end{array}$ & $\begin{array}{l}* / * \text { LTPA/WPA } \\
\text { ns/* LTPA/WPA } \\
* / \mathrm{ns} \text { LTPA/WPA } \\
\text { ns/* LTPA/WPA }\end{array}$ & $\begin{array}{l}\text { - LTPA and } \\
\text { recommended PA: } \\
\text { derived from } \\
\text { Behavioral Risk } \\
\text { Factor Surveillance } \\
\text { Survey Exercise } \\
\text { Module }\end{array}$ & $\begin{array}{l}\text { - } 76 \% \text { engaged in } \\
\text { LTPA in the past } \\
\text { month } \\
\text { - } 23 \% \text { engaged in } \\
\text { work-break PA } \\
\text { - } 22 \% \text { met the } \\
\text { recommended }\end{array}$ \\
\hline
\end{tabular}




\begin{tabular}{|c|c|c|c|c|c|c|c|}
\hline & & & & $\begin{array}{l}\text { outside work } \\
\text { Flexible work } \\
\text { schedule }\end{array}$ & $\begin{array}{l}\text { ns/ns } \\
\text { LTPA/WPA }\end{array}$ & $\begin{array}{l}\text { - Work-break PA: in } \\
\text { a usual week, do you } \\
\text { use your lunch or } \\
\text { other regular work } \\
\text { breaks to do PA or } \\
\text { exercise, such as } \\
\text { walking, aerobics, or } \\
\text { jogging, for at least } \\
10 \text { minutes at a time? }\end{array}$ & $\begin{array}{l}\text { levels of PA } \\
\text { - None of worksite } \\
\text { policies and } \\
\text { environments were } \\
\text { related to } \\
\text { recommended PA } \\
\text { - Reliability and } \\
\text { validity of } \\
\text { perceived worksite } \\
\text { policy and } \\
\text { environment } \\
\text { measure is } \\
\text { unknown }\end{array}$ \\
\hline $\begin{array}{l}\text { Payne et al. } \\
(2002)\end{array}$ & $\begin{array}{l}\mathrm{n}_{1}=241 \text { (baseline; } \\
70 \% \text { men) } \\
\text { Aged 16-24: } 9 \% \\
\text { Aged } 25-34: 26 \% \\
\text { Aged } 35-44: 32 \% \\
\text { Aged } 45-54: 26 \% \\
\text { Aged } \geq 55: 7 \% \\
\mathrm{n}_{2}=213 \text { (one week) } \\
\text { Setting: a large } \\
\text { computer company } \\
\text { in the UK }\end{array}$ & $\begin{array}{l}\text { Cross- } \\
\text { sectional }\end{array}$ & $\begin{array}{l}\text { TPB and } \\
\text { job strain } \\
\text { model }\end{array}$ & $\begin{array}{l}\text { Predict exercise } \\
\text { behavior from } \\
\text { TPB variables } \\
\text { Intention } \\
\text { Self-efficacy } \\
\text { Perceived control } \\
\text { Predict successful } \\
\text { implementation of } \\
\text { exercise intention } \\
\text { Self-efficacy } \\
\text { Job demands }\end{array}$ & $\begin{array}{l}\beta=.78 * * * \\
\beta=.12 * * \\
\mathrm{~ns}\end{array}$ & $\begin{array}{l}\text { - What forms of } \\
\text { exercise did you do } \\
\text { last week and how } \\
\text { many hours did you } \\
\text { devote to each? } \\
\text { - Exercise: taking } \\
\text { part in purposeful } \\
\text { activity which is } \\
\text { often structured and } \\
\text { pursued for health } \\
\text { and fitness benefits }\end{array}$ & $\begin{array}{l}\text { Intention, self- } \\
\text { efficacy, and } \\
\text { perceived control } \\
\text { explained } 71 \% \text { of } \\
\text { the variance in } \\
\text { exercise behavior }\end{array}$ \\
\hline $\begin{array}{l}\text { Payne et al. } \\
(2005)\end{array}$ & $\begin{array}{l}\mathrm{n}_{1}=331 \text { (baseline; } \\
70 \% \text { men) } \\
\text { Aged 16-24: } 7 \% \\
\text { Aged 25-34: } 21 \% \\
\text { Aged 35-44: } 32 \% \\
\text { Aged 45-54: } 33 \%\end{array}$ & $\begin{array}{l}\text { Cross- } \\
\text { sectional }\end{array}$ & $\begin{array}{l}\text { TPB and } \\
\text { job strain } \\
\text { model }\end{array}$ & $\begin{array}{l}\text { Intention (I) } \\
\text { PBC } \\
\text { Demands (D) } \\
\text { Control (C) } \\
D \times C \\
D \times I\end{array}$ & $\begin{array}{l}\text { ns } \\
\text { ns } \\
\text { ns } \\
\text { ns } \\
\text { ns } \\
\text { ns }\end{array}$ & $\begin{array}{l}\text { - What forms of } \\
\text { exercise did you do } \\
\text { last week and how } \\
\text { long did you devote } \\
\text { to each? } \\
\text { - Exercise: taking }\end{array}$ & $\begin{array}{l}\text { The final model } \\
\text { explained } 62 \% \text { of } \\
\text { the variance in } \\
\text { exercise behavior }\end{array}$ \\
\hline
\end{tabular}




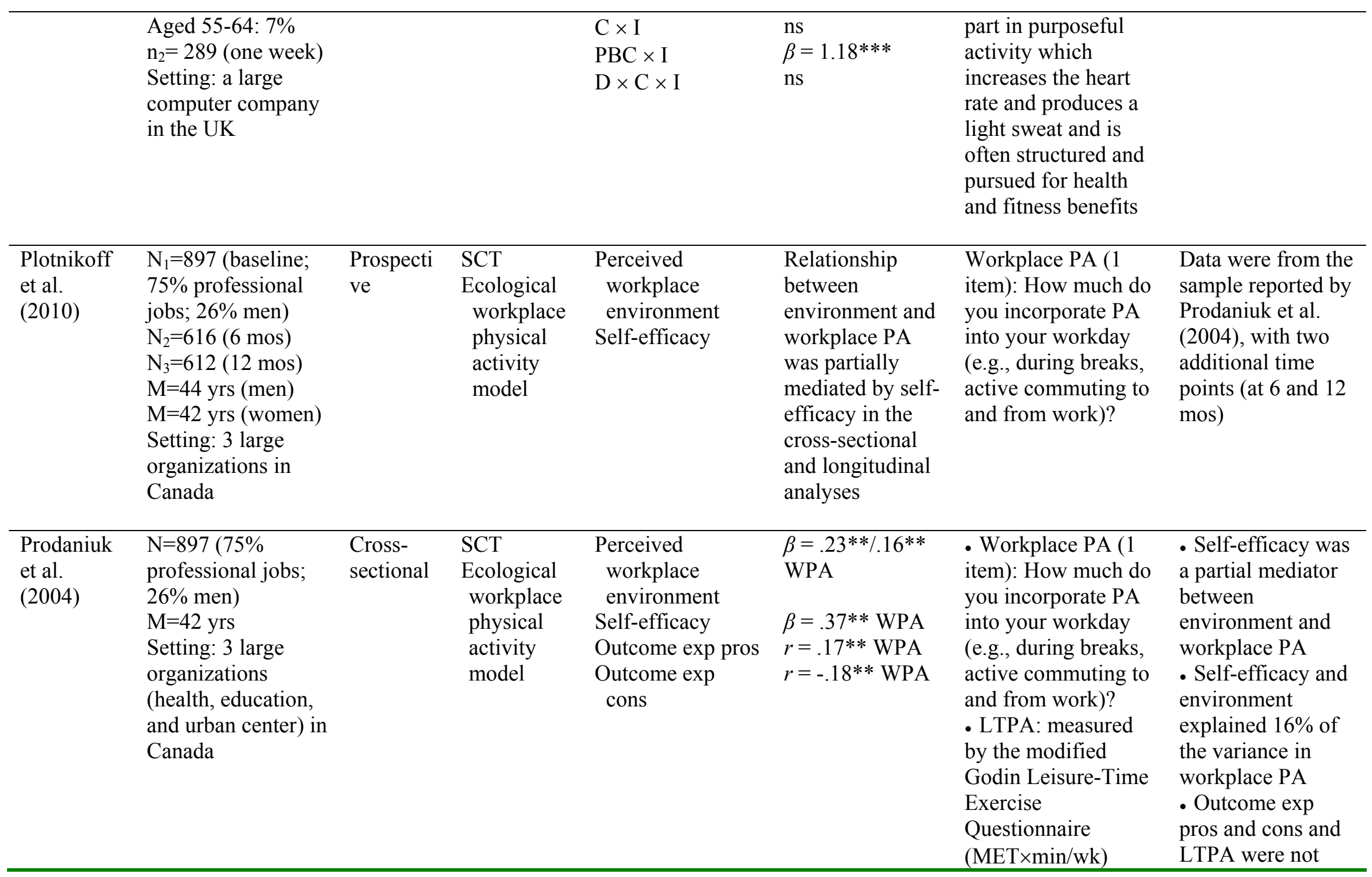


included in the

mediation analysis

due to small

relationship

Note. $\mathrm{PA}=$ physical activity; LTPA $=$ leisure-time physical activity; WPA $=$ work-break/workplace physical activity; TPB $=$ Theory of Planned Behavior; SCT = Social Cognitive Theory; $\exp =$ expectations; $\mathrm{PBC}=$ perceived behavioral control; $\mathrm{mos}=$ months; $\mathrm{wks}=$ weeks; $\mathrm{ns}=$ nonsignificant; $\mathrm{F} / \mathrm{M}=$ females/males; $\mathrm{OR}=$ odds ratio; $\mathrm{PR}=$ prevalence ratio.

${ }^{*} p<.05 .{ }^{* *} p<.01 .{ }^{* * *} p<.001$. 
Table 2.2 Characteristics and Main Findings of Studies Examining Relationships of Psychosocial Determinants with Physical Activity among White-Collar Workers

\begin{tabular}{|c|c|c|c|c|c|c|c|}
\hline Study & Sample & Design & $\begin{array}{l}\text { Theoretical } \\
\text { framework }\end{array}$ & $\begin{array}{c}\text { Determinant/ } \\
\text { Predictor }\end{array}$ & $\begin{array}{l}\text { Association with } \\
\text { PA/Exercise }\end{array}$ & $\begin{array}{c}\text { PA/Exercise } \\
\text { measurement }\end{array}$ & Notes \\
\hline $\begin{array}{l}\text { Chen \& } \\
\text { Chang } \\
(2004)\end{array}$ & $\begin{array}{l}\mathrm{N}=361 \text { women } \\
\text { Age=20-56 yrs } \\
\text { Setting: a bank in } \\
\text { Taiwan }\end{array}$ & $\begin{array}{l}\text { Cross- } \\
\text { sectional }\end{array}$ & $\begin{array}{l}\text { Self- } \\
\text { developed }\end{array}$ & $\begin{array}{l}\text { Benefits } \\
\text { Barriers } \\
\text { Self-efficacy } \\
\text { Body image } \\
\text { Social support } \\
\text { Enjoyment }\end{array}$ & $\begin{array}{l}\text { ns } \\
\operatorname{Exp}(\beta)=.94 \\
\operatorname{Exp}(\beta)=1.07 \\
\mathrm{~ns} \\
\operatorname{Exp}(\beta)=1.03 \\
\operatorname{Exp}(\beta)=1.10\end{array}$ & $\begin{array}{l}\text { Exercise behavior: } \\
\text { assessed low, } \\
\text { moderate, and high } \\
\text { intensity exercise } \\
\text { over the last } 6 \text { mos } \\
(\mathrm{MET} \times \mathrm{h} / \mathrm{wk})\end{array}$ & $\begin{array}{l}\text { Only } 8.6 \% \\
\text { engaged in regular } \\
\text { exercise }\end{array}$ \\
\hline
\end{tabular}

\begin{tabular}{|c|c|c|c|c|c|c|c|}
\hline $\begin{array}{l}\text { Jackson et } \\
\text { al. (2003) }\end{array}$ & $\begin{array}{l}\mathrm{N}_{1}=169 \text { (baseline; } \\
36 \% \text { men; } M=43 \\
\text { yrs) } \\
\mathrm{N}_{2}=85 \text { ( } 8 \text { wks; } 36 \% \\
\text { men; } \mathrm{M}=42 \mathrm{yrs}) \\
\text { Setting: a college in } \\
\text { England }\end{array}$ & $\begin{array}{l}\text { Prospecti } \\
\text { ve }\end{array}$ & $\begin{array}{l}\text { Extended } \\
\text { version of } \\
\text { the TPB }\end{array}$ & $\begin{array}{l}\text { Intention } \\
\text { Perceived control } \\
\text { Self-efficacy } \\
\text { Attitude } \\
\text { Subjective norm } \\
\text { Descriptive norm } \\
\text { Anticipated } \\
\text { affective reaction } \\
\text { Moral norm } \\
\text { Self-identity } \\
\text { Past behavior }\end{array}$ & $\begin{array}{l}\mathrm{ns} \\
\mathrm{ns} \\
\mathrm{ns} \\
\mathrm{ns} \\
\mathrm{ns} \\
\mathrm{ns} \\
\mathrm{ns} \\
\mathrm{ns} \\
\beta=.23 * \\
\beta=.57 * * *\end{array}$ & $\begin{array}{l}\text { - Modified version } \\
\text { of the Leisure-Time } \\
\text { Exercise } \\
\text { Questionnaire } \\
\text { (including activity at } \\
\text { work; a typical week } \\
\text { over the past } 8 \mathrm{wks} \text { ) } \\
\text { - Past PA: total PA } \\
\text { at baseline } \\
\text { - PA behavior: total } \\
\text { PA at } 8 \text { wks }\end{array}$ & $\begin{array}{l}\text { - } 5 \text { TPB variables } \\
\text { accounted for } 29 \% \\
\text { of the variance in } \\
\text { PA behavior } \\
\text { - } 5 \text { TPB variables } \\
\text { and } 5 \text { additional } \\
\text { variables explained } \\
64 \% \text { of the } \\
\text { variance in PA } \\
\text { behavior }\end{array}$ \\
\hline $\begin{array}{l}\text { Kaewthum } \\
\text { manukul et } \\
\text { al. (2006) }\end{array}$ & $\begin{array}{l}\mathrm{N}=970 \text { female } \\
\text { nurses } \\
\mathrm{M}=33 \text { yrs } \\
\text { Setting: a hospital } \\
\text { in Thailand }\end{array}$ & $\begin{array}{l}\text { Cross- } \\
\text { sectional }\end{array}$ & $\begin{array}{l}\text { SCT } \\
\text { HPM }\end{array}$ & $\begin{array}{l}\text { Self-efficacy } \\
\text { Barriers } \\
\text { Benefits } \\
\text { Social support } \\
\text { - family members } \\
\text { - friends } \\
\text { Job demands } \\
\text { - psychological } \\
\text { - physical } \\
\text { Motivation }\end{array}$ & $\begin{array}{l}\beta=.06 * * * \\
\beta=-.06 * * \\
\beta=-.02 * \\
\beta=.06 * * \\
\text { ns } \\
\text { ns } \\
\text { ns } \\
\beta=.01 * *\end{array}$ & $\begin{array}{l}\text { Nurses' Health } \\
\text { Study II Activity and } \\
\text { Inactivity } \\
\text { Questionnaire } \\
\text { (weekly recreational } \\
\text { PA and selected } \\
\text { routines of daily } \\
\text { living over the past } \\
\text { month) }\end{array}$ & $\begin{array}{l}\text { - } 67.5 \% \text { met } \mathrm{PA} \\
\text { recommendation } \\
(\geq 21 \mathrm{MET} \times \mathrm{h} / \mathrm{wk}) \\
\text { - Final model } \\
\text { explained } 18 \% \text { of } \\
\text { the variance in PA }\end{array}$ \\
\hline
\end{tabular}




\begin{tabular}{|c|c|c|c|c|c|c|c|}
\hline $\begin{array}{l}\text { Kao et al. } \\
(2002)\end{array}$ & $\begin{array}{l}\mathrm{N}=193 \text { sedentary } \\
\text { workers } \\
\mathrm{n}_{1}=133 \\
\text { (experimental; } 68 \% \\
\text { men; } \mathrm{M}=47 \mathrm{yrs} \text { ) } \\
\mathrm{n}_{2}=60 \text { (control; } \\
72 \% \text { men; } \mathrm{M}=44 \\
\text { yrs) } \\
\text { Setting: a telecom } \\
\text { company in Taiwan }\end{array}$ & $\begin{array}{l}\text { Cross- } \\
\text { sectional } \\
\text { data from } \\
\text { a quasi- } \\
\text { experime } \\
\text { ntal } \\
\text { design }\end{array}$ & TTM & $\begin{array}{l}\text { Benefits } \\
\text { Barriers } \\
\text { Self-efficacy }\end{array}$ & $\begin{array}{l}\mathrm{ns} \\
\mathrm{F}=8.5 * * * \\
(1)^{\dagger}>\text { (5) } \\
\text { (2) }>\text { (4) } \\
\text { (2) }>\text { (5) } \\
\mathrm{F}=11.5 * * * \\
\text { (5) }>\text { (1) } \\
\text { (5) }>\text { (2) } \\
\text { (5) }>\text { (3) }\end{array}$ & $\begin{array}{l}5 \text { stages of exercise } \\
\text { behavior }{ }^{\dagger} \text { : } \\
\text { (1) Precontemplation } \\
\text { (2) Contemplation } \\
\text { (3) Preparation } \\
\text { (4) Action } \\
\text { (5) Maintenance }\end{array}$ & $\begin{array}{l}\text { Results presented } \\
\text { here used data } \\
\text { from pretest of the } \\
\text { experimental group }\end{array}$ \\
\hline $\begin{array}{l}\text { Lee et al. } \\
(2005)\end{array}$ & $\begin{array}{l}\mathrm{N}=300 \text { female } \\
\text { nurses } \\
\mathrm{M}=31 \text { yrs } \\
\text { Setting: } 4 \text { hospitals } \\
\text { in Taiwan }\end{array}$ & $\begin{array}{l}\text { Cross- } \\
\text { sectional }\end{array}$ & None & $\begin{array}{l}\text { Benefits } \\
\text { Barriers } \\
\text { Self-efficacy }\end{array}$ & $\begin{array}{l}\mathrm{ns} \\
\mathrm{ns} \\
\beta=.25 * * *\end{array}$ & $\begin{array}{l}\text { 3-day PA record } \\
\text { - } 1 \text { working day and } \\
2 \text { non-working days } \\
\text { - Including all daily } \\
\text { activities } \\
\text { - Activities were } \\
\text { converted into } \\
\text { energy expenditure } \\
\text { (EE, } \mathrm{kcal} / \mathrm{kg} / \text { day) }\end{array}$ & $\begin{array}{l}\text { - Mean EE for all } \\
\text { PA (kcal/kg/day) } \\
\text { on working days } \\
\text { (56) was greater } \\
\text { than that on non- } \\
\text { working days (43) } \\
\text { - Self-efficacy, } \\
\text { marital status, and } \\
\text { education } \\
\text { explained } 11 \% \text { of } \\
\text { the variance in PA } \\
\text { on working and } \\
\text { non-working days }\end{array}$ \\
\hline $\begin{array}{l}\text { Nishida et } \\
\text { al. (2004) }\end{array}$ & $\begin{array}{l}\mathrm{N}=273 \text { men }(63 \% \\
\text { office workers }) \\
\mathrm{M}=33 \text { yrs } \\
\text { Setting: } 5 \\
\text { manufacturing } \\
\text { companies in Japan }\end{array}$ & $\begin{array}{l}\text { Cross- } \\
\text { sectional }\end{array}$ & TTM & $\begin{array}{l}\text { Benefits } \\
\text { - psychological } \\
\text { - health } \\
\text { - social } \\
\text { - weight control } \\
\text { - vital } \\
\text { Barriers }\end{array}$ & $\begin{array}{l}\mathrm{F}=8.8 * * \\
\mathrm{~ns} \\
\mathrm{~F}=8.5 * * \\
\mathrm{~ns} \\
\mathrm{~F}=4.4 * *\end{array}$ & $\begin{array}{l}\text { 8-item current PA } \\
\text { level was used and } \\
\text { answers were } \\
\text { converted to } 5 \text { stages } \\
\text { of PA: } \\
\text { (1) Precontemplation } \\
\text { (2) Contemplation }\end{array}$ & $\begin{array}{l}\text { Only } 8 \% \text { were in } \\
\text { the action or } \\
\text { maintenance stages } \\
\text { of PA (met the } \\
\text { recommended } \\
\text { levels of PA) }\end{array}$ \\
\hline
\end{tabular}




\begin{tabular}{|c|c|c|c|c|c|c|c|}
\hline & & & & $\begin{array}{l}\text { - physical } \\
\text { - specific } \\
\text { obstacles } \\
\text { - time } \\
\text { Self-efficacy }\end{array}$ & $\begin{array}{l}\mathrm{F}=5.0 * * \\
\mathrm{~ns} \\
\mathrm{~ns}(p=.06) \\
\mathrm{F}=23.6^{* *}\end{array}$ & $\begin{array}{l}\text { (3) Preparation } \\
\text { (4) Action } \\
\text { (5) Maintenance }\end{array}$ & \\
\hline $\begin{array}{l}\text { Purath } \\
(2006)\end{array}$ & $\begin{array}{l}\mathrm{N}=373 \text { women } \\
\mathrm{n}_{1}=86 \text { active } \\
\mathrm{n}_{2}=287 \text { inactive } \\
\mathrm{M}=44 \text { yrs } \\
\text { Setting: a } \\
\text { university in the US }\end{array}$ & $\begin{array}{l}\text { Cross- } \\
\text { sectional }\end{array}$ & TTM & $\begin{array}{l}\text { Self-efficacy } \\
\text { (overall) } \\
\text { - raining/snowing } \\
\text { Pros (overall) } \\
\text { - less stressed } \\
\text { - positive outlook } \\
\text { Cons (overall) } \\
\text { - bad weather } \\
\text { - time } \\
\text { - tired }\end{array}$ & $\begin{array}{l}\mathrm{OR}=1.10 * * \\
\mathrm{OR}=1.37 * \\
\mathrm{~ns} \\
\mathrm{OR}=1.95 * \\
\mathrm{OR}=1.94 * \\
\mathrm{~ns} \\
\mathrm{OR}=.64 * \\
\mathrm{OR}=.60 * \\
\mathrm{OR}=.66^{*}\end{array}$ & $\begin{array}{l}\text { Stage of change: } \\
\text { Current Physical } \\
\text { Activity Status ( } 8 \\
\text { stages) was used to } \\
\text { classify cases as } \\
\text { physically active (1- } \\
\text { 4) or inactive (5-8) }\end{array}$ & $\begin{array}{l}\text { - } 23 \% \text { met the } \\
\text { recommended } \\
\text { levels of PA } \\
\text { - Physically active } \\
\text { women were more } \\
\text { likely to have a } \\
\text { past history of } \\
\text { sustained PA ( } \geq 6 \\
\text { mos) than inactive } \\
\text { women }\end{array}$ \\
\hline $\begin{array}{l}\text { Sassen et } \\
\text { al. (2010) }\end{array}$ & $\begin{array}{l}\mathrm{N}=1298 \text { police } \\
(67 \% \text { men }) \\
\text { Age }=18-62 \text { yrs } \\
\text { Setting: a police } \\
\text { department in } \\
\text { Netherlands }\end{array}$ & $\begin{array}{l}\text { Cross- } \\
\text { sectional }\end{array}$ & TPB & $\begin{array}{l}\text { Attitude } \\
\text { Subjective norm } \\
\text { Descriptive norm } \\
\text { Perceived control } \\
\text { Self-efficacy } \\
\text { Intention } \\
\text { Barriers }\end{array}$ & $\begin{array}{l}\text { ns } \\
\text { ns } \\
\beta=.08 * * \\
\text { ns } \\
\beta=.24 * * * \\
\beta=.47 * * * \\
\beta=-.10 * * *\end{array}$ & $\begin{array}{l}2 \text { items assessed PA: } \\
\text { - Are you physically } \\
\text { active for at least } 60 \\
\text { minutes a day? } \\
\text { - Do you think you } \\
\text { engage in adequate } \\
\text { PA?' }\end{array}$ & $\begin{array}{l}\text { Regression } \\
\text { analyses were used } \\
\text { for people with } \geq 1 \\
\text { CV risk factors } \\
\text { ( } \mathrm{n}=989 \text { ); TPB } \\
\text { explained } 52 \% \text { of } \\
\text { the variance in PA } \\
\text { behavior }\end{array}$ \\
\hline $\begin{array}{l}\text { Steinhardt } \\
\text { \& Dishman } \\
(1989)\end{array}$ & $\begin{array}{l}\mathrm{N}=968 \text { primarily } \\
\text { white upper middle } \\
\text { class workers } \\
\mathrm{M}=38.5 \text { yrs } \\
\text { Setting: an } \\
\text { American oil } \\
\text { company }\end{array}$ & $\begin{array}{l}\text { Cross- } \\
\text { sectional }\end{array}$ & None & $\begin{array}{l}\text { Outcome- } \\
\text { expectancy values } \\
\text { - psychologic } \\
\text { - body image/ } \\
\text { health } \\
\text { - social } \\
\text { - competitive }\end{array}$ & $\begin{array}{l}\beta=.14 * * \\
\beta=.12 * * \\
\beta=-.08^{*} \\
\text { ns }\end{array}$ & $\begin{array}{l}\text { Regular participation } \\
\text { in the onsite fitness } \\
\text { program: } \\
\text { On the average, how } \\
\text { often, per month, } \\
\text { have you used the } \\
\text { fitness center in the }\end{array}$ & $\begin{array}{l}\text { - Outcome- } \\
\text { expectancy values } \\
\text { and barriers } \\
\text { explained } 12 \% \text { of } \\
\text { the variance in } \\
\text { participation in the } \\
\text { onsite fitness }\end{array}$ \\
\hline
\end{tabular}




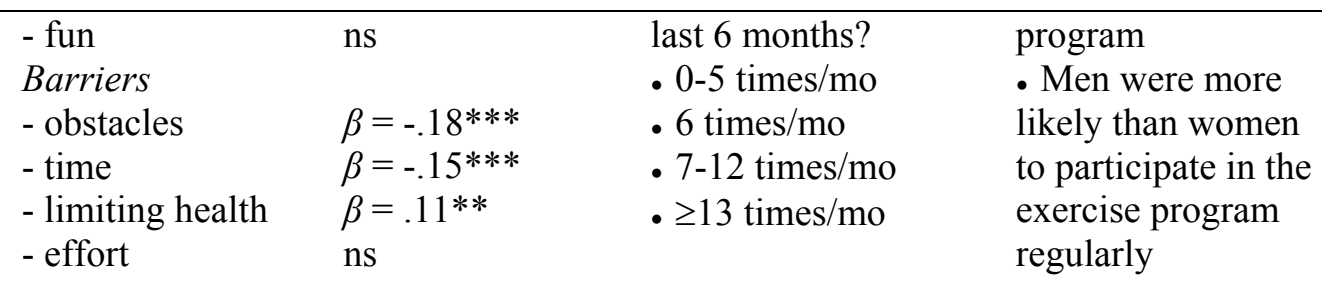

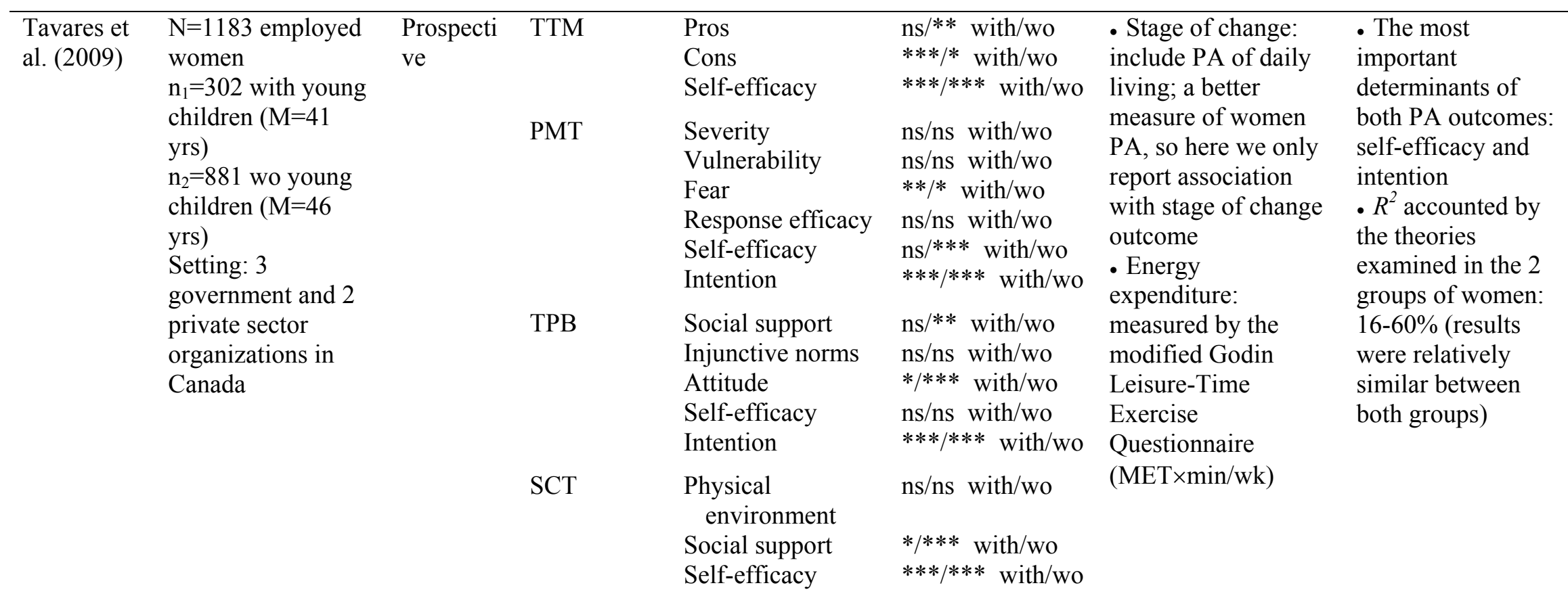

Note. PA = physical activity; LTPA = leisure-time physical activity; TPB = Theory of Planned Behavior; SCT = Social Cognitive

Theory; HPM = Health Promotion Model; TTM = Transtheoretical model; PMT = Protection Motivation Theory; CV = cardiovascular; mos $=$ months; $w k s=$ weeks; $w o=$ without; $n s=$ nonsignificant; $\mathrm{OR}=$ odds ratio.

$\dagger 5$ stages of exercise behavior: (1) = Precontemplation, (2) = Contemplation, (3) = Preparation, (4) = Action, (5) = Maintenance.

${ }^{*} p<.05 .{ }^{* *} p<.01 .{ }^{* * *} p<.001$. 
Table 2.3 Association of Job Strain Variables with Physical Activity among White-Collar Workers

\begin{tabular}{|c|c|c|c|c|c|c|}
\hline \multirow[b]{2}{*}{ Variable } & \multicolumn{3}{|c|}{ Significant $(p<.05)$} & \multicolumn{3}{|c|}{ Nonsignificant } \\
\hline & Women & Men & Both Sexes & Women & Men & Both Sexes \\
\hline Job demands & Brisson (2000) & & Payne (2002) & $\begin{array}{l}\text { Hellerstedt (1997) } \\
\text { Kouvonen (2005) } \\
\text { Kaewthummanukul (2006) }\end{array}$ & $\begin{array}{l}\text { Brisson (2000) } \\
\text { Hellerstedt (1997) } \\
\text { Kouvonen (2005) } \\
\text { Landsbergis (1998) }\end{array}$ & Payne (2005) \\
\hline Job control & $\begin{array}{l}\text { Hellerstedt (1997) } \\
\text { Kouvonen (2005) }\end{array}$ & $\begin{array}{l}\text { Brisson (2000) } \\
\text { Hellerstedt (1997) } \\
\text { Kouvonen (2005) }\end{array}$ & & Brisson (2000) & Landsbergis (1998) & $\begin{array}{l}\text { Payne (2002) } \\
\text { Payne (2005) }\end{array}$ \\
\hline High strain job & $\begin{array}{l}\text { Hellerstedt (1997) } \\
\text { Kouvonen (2005) } \\
\text { Lallukka (2008) }\end{array}$ & $\begin{array}{l}\text { Brisson (2000) } \\
\text { Hellerstedt (1997) } \\
\text { Kouvonen (2005) } \\
\text { Lallukka (2008) }\end{array}$ & & $\begin{array}{l}\text { Brisson (2000) } \\
\text { Lallukka (2004) }\end{array}$ & $\begin{array}{l}\text { Lallukka (2004) } \\
\text { Landsbergis (1998) }\end{array}$ & \\
\hline Passive job & $\begin{array}{l}\text { Hellerstedt (1997) } \\
\text { Kouvonen (2005) } \\
\text { Lallukka (2008) }\end{array}$ & $\begin{array}{l}\text { Brisson (2000) } \\
\text { Gimeno (2009) } \\
\text { Hellerstedt (1997) } \\
\text { Kouvonen (2005) } \\
\text { Lallukka (2008) }\end{array}$ & & $\begin{array}{l}\text { Brisson }(2000) \\
\text { Gimeno (2009) } \\
\text { Lallukka (2004) }\end{array}$ & Lallukka (2004) & \\
\hline Active job & Hellerstedt (1997) & Kouvonen (2005) & & $\begin{array}{l}\text { Brisson (2000) } \\
\text { Kouvonen (2005) } \\
\text { Lallukka (2004) } \\
\text { Lallukka (2008) }\end{array}$ & $\begin{array}{l}\text { Brisson (2000) } \\
\text { Hellerstedt (1997) } \\
\text { Lallukka (2004) } \\
\text { Lallukka (2008) }\end{array}$ & \\
\hline Low strain job & Hellerstedt (1997) & Hellerstedt (1997) & & Lallukka (2004) & Lallukka (2004) & \\
\hline
\end{tabular}


Table 2.4 Association of Psychosocial Variables with Physical Activity among White-Collar Workers

\begin{tabular}{|c|c|c|c|c|c|}
\hline \multirow[b]{2}{*}{ Variable } & \multicolumn{3}{|c|}{ Significant $(p<.05)$} & \multicolumn{2}{|c|}{ Nonsignificant } \\
\hline & Women & Men & Both Sexes & Women & Both Sexes \\
\hline Body image & & & & Chen (2004) & \\
\hline Descriptive norm & & & Sassen (2010) & & Jackson (2003) \\
\hline Intention & Tavares (2009) & & $\begin{array}{l}\text { Payne (2002) } \\
\text { Sassen (2010) }\end{array}$ & & $\begin{array}{l}\text { Jackson (2003) } \\
\text { Payne (2005) }\end{array}$ \\
\hline Moral norm & & & & & Jackson (2003) \\
\hline Motivation & Kaewthummanukul (2006) & & & & \\
\hline $\begin{array}{l}\text { Outcome expectations } \\
\text { Anticipated affective } \\
\text { reaction }\end{array}$ & & & & & Jackson (2003) \\
\hline Attitude & Tavares (2009) & & & & $\begin{array}{l}\text { Jackson (2003) } \\
\text { Sassen (2010) }\end{array}$ \\
\hline Barriers/Cons & $\begin{array}{l}\text { Chen (2004) } \\
\text { Kaewthummanukul (2006) } \\
\text { Purath (2006) } \\
\text { Tavares (2009) }\end{array}$ & Nishida (2004) & $\begin{array}{l}\text { Kao (2002) } \\
\text { Prodaniuk (2004) } \\
\text { Sassen (2010) } \\
\text { Steinhardt (1989) }\end{array}$ & Lee (2005) & \\
\hline Benefits/Pros & $\begin{array}{l}\text { Kaewthummanukul (2006) } \\
\text { Purath (2006) } \\
\text { Tavares (2009) }\end{array}$ & Nishida (2004) & $\begin{array}{l}\text { Prodaniuk (2004) } \\
\text { Steinhardt (1989) }\end{array}$ & $\begin{array}{l}\text { Chen (2004) } \\
\text { Lee (2005) }\end{array}$ & Kao (2002) \\
\hline Enjoyment & Chen (2004) & & & & \\
\hline Fear & Tavares (2009) & & & & \\
\hline Response efficacy & & & & Tavares (2009) & \\
\hline Severity & & & & Tavares (2009) & \\
\hline Vulnerability & & & & Tavares (2009) & \\
\hline Perceived control/ & & & & & Jackson (2003) \\
\hline
\end{tabular}




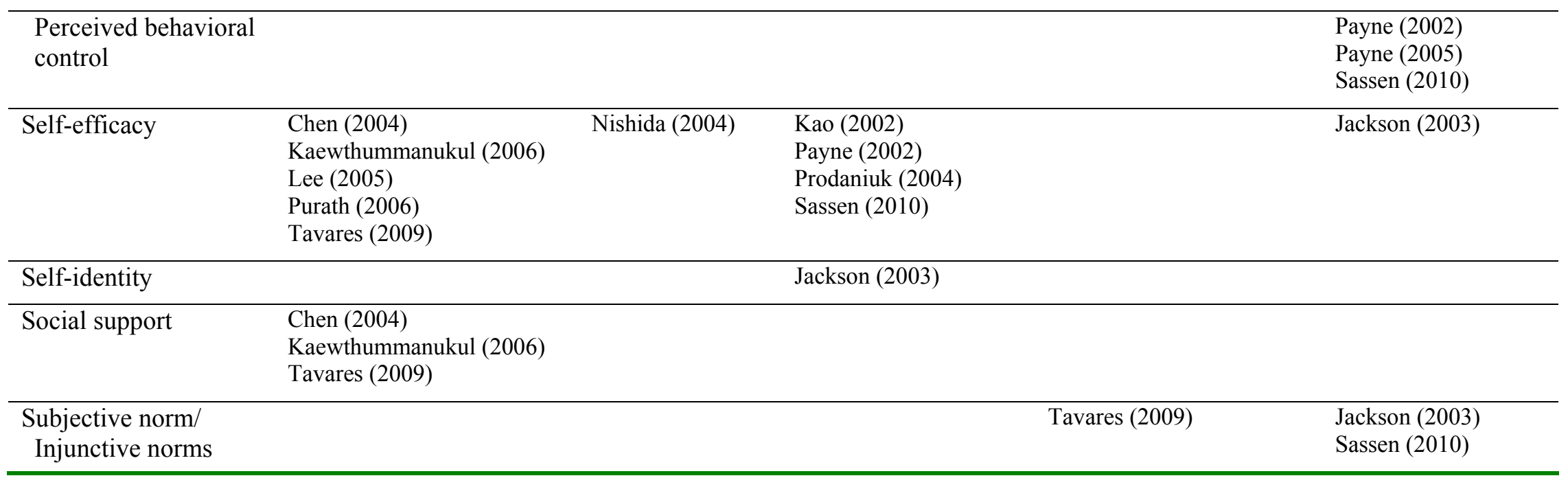




\section{References}

Andersen, L. B., Schnohr, P., Schroll, M., \& Hein, H. O. (2000). All-cause mortality associated with physical activity during leisure time, work, sports, and cycling to work. Archives of Internal Medicine, 160(11), 1621-1628.

Bandura, A. (1986). Social foundations of thought and action: A social cognitive theory. Englewood Cliffs, N.J.: Prentice-Hall.

Bandura, A. (2004). Health promotion by social cognitive means. Health Education \& Behavior, 31(2), 143-164.

Baranowski, T., Cullen, K. W., Nicklas, T., Thompson, D., \& Baranowski, J. (2003). Are current health behavioral change model helpful in guiding prevention of weight gain efforts? Obesity Research, 11 Suppl, 23S-43S.

Baranowski, T., Perry, C. L., \& Parcel, G. S. (2002). How individuals, environments, and health behavior interact: Social Cognitive Theory. In K. Glanz, B. K. Rimer \& F. M. Lewis (Eds.), Health Behavior and Health Education: Theory, Research and Practice (3rd ed., pp. 165-184). San Fransisco: Jossey-Bass.

Bennett, J. A. (2000). Focus on research methods. Mediator and moderator variables in nursing research: Conceptual and statistical differences. Research in Nursing \& Health, 23(4), 415-420.

Brisson, C., Larocque, B., Moisan, J., Vezina, M., \& Dagenais, G. R. (2000). Psychosocial factors at work, smoking, sedentary behavior, and body mass index: A prevalence study among 6995 white collar workers. Journal of Occupational and Environmental Medicine, 42(1), 40-46.

Brug J., Oenema, A., \& Ferreira, I. (2005). Theory, evidence and intervention mapping to improve behavior nutrition and physical activity interventions. International Journal of Behavioral Nutrition and Physical Activity, 2(1), 2.

Chen, C. M., \& Chang, M. (2004). Exercise behavior and related factors in career women -- the case of a bank in Taipei City. Journal of Nursing Research, 12(3), 180-189.

Chen, J. J., Wang, T. M., Lan, C. F., Huang, Y. G., Chiang, I. T., Wu, S. K. et al., (2008). Healthy lifestyle -- Physical activity. In K. N. Kuo (Ed.), Healthy People 2020 (pp.183-224). Taipei: National Health Research Institutes. (in Chinese)

Dishman, R. K., Oldenburg, B., O’Neal, H., \& Shephard, R. J. (1998). Worksite physical activity interventions. American Journal of Preventive Medicine, 15(4), 344-361.

Dodson, E. A., Lovegreen, S. L., Elliott, M. B., Haire-Joshu, D., \& Brownson, R. C. (2008). Worksite policies and environments supporting physical activity in midwestern communities. American Journal of Health Promotion, 23(1), 51-55. 
Engbers, L. H., van Poppel, M. N., Chin, A. P. M. J., \& van Mechelen, W. (2005). Worksite health promotion programs with environmental changes: A systematic review. American Journal of Preventive Medicine, 29(1), 61-70.

Gimeno, D., Elovainio, M., Jokela, M., De Vogli, R., Marmot, M. G., \& Kivimaki, M. (2009). Association between passive jobs and low levels of leisure-time physical activity: The Whitehall II cohort study. Occupational and Environmental Medicine, 66(11), 772-776.

Hamilton, M. T., Hamilton, D. G., \& Zderic, T. W. (2007). Role of low energy expenditure and sitting in obesity, metabolic syndrome, type 2 diabetes, and cardiovascular disease. Diabetes, 56(11), 2655-2667.

Hellerstedt, W. L., \& Jeffery, R. W. (1997). The association of job strain and health behaviours in men and women. International Journal of Epidemiology, 26(3), 575-583.

Jackson, C., Smith, R. A., \& Conner, M. (2003). Applying an extended version of the theory of planned behaviour to physical activity. Journal of Sports Sciences, 21(2), 119-133.

Kaewthummanukul, T., \& Brown, K. C. (2006). Determinants of employee participation in physical activity: Critical review of the literature. AAOHN Journal, 54(6), 249261.

Kaewthummanukul, T., Brown, K. C., Weaver, M. T., \& Thomas, R. R. (2006). Predictors of exercise participation in female hospital nurses. Journal of Advanced Nursing, 54(6), 663-675.

Kao, Y. H., Lu, C. M., \& Huang, Y. C. (2002). Impact of a transtheoretical model on the psychosocial factors affecting exercise among workers. Journal of Nursing Research, 10(4), 303-310.

Karasek, R. (1979). Job demands, job decision latitude, and mental strain: Implications for job redesign. Administrative Science Quarterly, 24(2), 285-308.

Karasek, R., Brisson, C., Kawakami, N., Houtman, I., Bongers, P., \& Amick, B. (1998). The Job Content Questionnaire (JCQ): An instrument for internationally comparative assessments of psychosocial job characteristics. Journal of Occupational Health Psychology, 3(4), 322-355.

Kouvonen, A., Kivimaki, M., Elovainio, M., Virtanen, M., Linna, A., \& Vahtera, J. (2005). Job strain and leisure-time physical activity in female and male public sector employees. Preventive Medicine, 41(2), 532-539.

Kremers, S. P., de Bruijn, G. J., Visscher, T. L., van Mechelen, W., de Vries, N. K., \& Brug, J. (2006). Environmental influences on energy balance-related behaviors: A 
dual-process view. International Journal of Behavioral Nutrition and Physical Activity, 3, 9.

Kruger J, Yore, M. M., Bauer, D. R., \& Kohl, H. W. (2007). Selected barriers and incentives for worksite health promotion services and policies. American Journal of Health Promotion, 21(5), 439-447.

Lallukka, T., Lahelma, E., Rahkonen, O., Roos, E., Laaksonen, E., Martikainen, P., et al. (2008). Associations of job strain and working overtime with adverse health behaviors and obesity: Evidence from the Whitehall II Study, Helsinki Health Study, and the Japanese Civil Servants Study. Social Science \& Medicine, 66(8), 1681-1698.

Lallukka, T., Sarlio-Lahteenkorva, S., Roos, E., Laaksonen, M., Rahkonen, O., \& Lahelma, E. (2004). Working conditions and health behaviours among employed women and men: The Helsinki health study. Preventive Medicine, 38(1), 48-56.

Landsbergis, P. A., Schnall, P. L., Deitz, D. K., Warren, K., Pickering, T. G., \& Schwartz, J. E. (1998). Job strain and health behaviors: Results of a prospective study. American Journal of Health Promotion, 12(4), 237-245.

Lee, Y. S., Huang, Y. C., \& Kao, Y. H. (2005). Physical activities and correlates of clinical nurses in Taipei municipal hospitals. Journal of Nursing Research, 13(4), 281-292.

Lucove, J. C., Huston, S. L., \& Evenson, K. R. (2007). Workers' perceptions about worksite policies and environments and their association with leisure-time physical activity. American Journal of Health Promotion, 21(3), 196-200.

Marcus, B. H., Williams, D. M., Dubbert, P. M., Sallis, J. F., King, A. C., Yancey, A. K., et al. (2006). Physical activity intervention studies: What we know and what we need to know: A scientific statement from the American Heart Association Council on Nutrition, Physical Activity, and Metabolism (Subcommittee on Physical Activity); Council on Cardiovascular Disease in the Young; and the Interdisciplinary Working Group on Quality of Care and Outcomes Research. Circulation, 114(24), 2739-2752.

Matson-Koffman, D. M., Brownstein, J. N., Neiner, J. A., \& Greaney, M. L. (2005). A site-specific literature review of policy and environmental interventions that promote physical activity and nutrition for cardiovascular health: What works? American Journal of Health Promotion, 19(3), 167-193.

Morris, J. N., Heady, J. A., Raffle, P. A., Roberts, C. G., \& Parks, J. W. (1953a). Coronary heart-disease and physical activity of work. Lancet, 265(6795), 10531057; contd.

Morris, J. N., Heady, J. A., Raffle, P. A., Roberts, C. G., \& Parks, J. W. (1953b). Coronary heart-disease and physical activity of work. Lancet, 265(6796), 1111- 
1120; concl.

Nishida, Y., Suzuki, H., Wang, D. H., \& Kira, S. (2004). Psychological correlates of physical activity and exercise in Japanese male employees. International Journal of Sport and Health Science, 2, 136-144.

Patel, A. V., Bernstein, L., Deka, A., Feigelson, H. S., Campbell, P. T., Gapstur, S. M., et al. (2010). Leisure time spent sitting in relation to total mortality in a prospective cohort of US adults. American Journal of Epidemiology, 172(4), 419-429.

Payne, N., Jones, F., \& Harris, P. (2002). The impact of working life on health behavior: The effect of job strain on the cognitive predictors of exercise. Journal of Occupational Health Psychology, 7(4), 342-353.

Payne, N., Jones, F., \& Harris, P. R. (2005). The impact of job strain on the predictive validity of the theory of planned behaviour: An investigation of exercise and healthy eating. British Journal of Health Psychology, 10(Pt 1), 115-131.

Plotnikoff, R. C., Pickering, M. A., Flaman, L. M., \& Spence, J. C. (2010). The role of self-efficacy on the relationship between the workplace environment and physical activity: A longitudinal mediation analysis. Health Education \& Behavior, 37(2), 170-185.

Prodaniuk, T. R., Plotnikoff, R. C., Spence, J. C., \& Wilson, P. M. (2004). The influence of self-efficacy and outcome expectations on the relationship between perceived environment and physical activity in the workplace. International Journal of Behavioral Nutrition and Physical Activity, 1(1), 7.

Pronk, N. P. (2009). Physical activity promotion in business and industry: Evidence, context, and recommendations for a national plan. Journal of Physical Activity \& Health, 6 Suppl 2, S220-235.

Proper, K. I., Koning, M., van der Beek, A. J., Hildebrandt, V. H., Bosscher, R. J., van Mechelen, W. (2003). The effectiveness of worksite physical activity programs on physical activity, physical fitness, and health. Clinical Journal of Sport Medicine, 13(2), 106-117.

Purath, J. (2006). Comparison of the traits of physically active and inactive women. Journal of the American Academy of Nurse Practitioners, 18(5), 234-240.

Sallis, J. F., \& Owen, N. (2002). Ecological models of health behavior. In K. Glanz, F. M. Lewis, \& B. K. Rimer (Eds.), Health behavior and health education: Theory, research, and practice (3rd ed., pp. 462-484). San Francisco: John Wiley \& Sons.

Sassen, B., Kok, G., Schaalma, H., Kiers, H., \& Vanhees, L. (2010). Cardiovascular risk profile: Cross-sectional analysis of motivational determinants, physical fitness and physical activity. BMC Public Health, 10, 592. 
Spence, J. C., \& Lee, R. E. (2003). Toward a comprehensive model of physical activity. Psychology of Sport and Exercise, 4(1), 7-24.

Steinhardt, M. A., \& Dishman, R. K. (1989). Reliability and validity of expected outcomes and barriers for habitual physical activity. Journal of Occupational Medicine, 31(6), 536-546.

Taiwan 1111 Job Bank. (2007). Physically inactive group in the workplace -- A survey of physical activity habits among office workers. Retrieved February 5, 2011, from http://project.1111.com.tw/zone/pr/headline.asp?autono=1702 (in Chinese)

Taiwan Department of Health. (2010). 2009 Taiwan public health report. Taipei: Author. (in Chinese)

Tavares, L. S., Plotnikoff, R. C., \& Loucaides, C. (2009). Social-cognitive theories for predicting physical activity behaviours of employed women with and without young children. Psychology, Health \& Medicine, 14(2), 129-142.

Taylor, H. L., Klepetar, E., Keys, A., Parlin, W., Blackburn, H., \& Puchner, T. (1962). Death rates among physically active and sedentary employees of the railroad industry. American Journal of Public Health and the Nation's Health, 52, 1697 1707.

U.S. Department of Health and Human Services. (2000). Healthy people 2010: Understanding and improving health. $2^{\text {nd }}$ ed. Washington, DC: U.S. Government Printing Office.

U.S. Department of Health and Human Services. (2008). 2008 Physical activity guidelines for Americans. Retrieved March 1, 2009 from http://www.health.gov/PAGuidelines/pdf/paguide.pdf

Williams, D. M., Anderson, E. S., \& Winett, R. A. (2005). A review of the outcome expectancy construct in physical activity research. Annals of Behavioral Medicine, 29(1), 70-79.

World Health Organization. (2002). The world health report 2002: Reducing risks, promoting healthy life. Retrieved March 18, 2008, from http://www.who.int/whr/2002/en/whr02_en.pdf

World Health Organization. (2008). Preventing noncommunicable diseases in the workplace through diet and physical activity: WHO/World Economic Forum report of a joint event. Retrieved February 1, 2009, from http://www.weforum.org/pdf/Wellness/WHOWEF_report.pdf 


\title{
CHAPTER 3
}

\section{Reliability and Validity of the Chinese Version of the Perceived Workplace Environment Scale in Taiwanese Information Technology Professionals}

\begin{abstract}
The six-item Perceived Workplace Environment Scale (PWES) is a recently developed instrument designed to measure the perceptions of workplace environments that support physical activity. This paper describes the translation process and the psychometric testing of the Chinese version of the PWES (PWES-C). Psychometric properties were examined by a survey of 466 male and 109 female Taiwanese information technology professionals. Internal consistency reliability was high (Cronbach's $\alpha=.88$ ), and only minor ceiling and floor effects were observed for the PWES-C. Content validity was supported by a panel of experts. Construct validity was supported by examining the factor structure of the PWES-C using confirmatory factor analysis, and the results suggest a single factor structure. The PWES-C is a reliable and valid measure of perceived workplace environment in information technology professionals.
\end{abstract}




\section{Introduction}

There are four opportunities for people to remain physically active in their daily lives, such as having a job that involves significant walking or manual labor, walking or cycling to work, household work, or leisure time participation in sports or active activities (World Health Organization [WHO], 2002). However, living in an urbanized and mechanized society has greatly reduced physical survival demands. Contemporary economy requires fewer workers performing hard physical labor, and more workers spend their day sitting and doing light work. Changes to living and working environments have considerably contributed to lower physical activity levels among workers (van Poppel \& Engbers, 2009).

Current approaches to worksite health promotion focus on creating supportive environments to facilitate health behavior change (Golaszewski, Allen, \& Edington, 2008). The ecological model for health promotion (McLeroy, Bibeau, Steckler, \& Glanz, 1988) includes five elements that influence behavior: (a) intrapersonal factors, (b) interpersonal processes and primary groups, (c) institutional factors, (d) community factors, and (e) public policy. To this model, Sallis and Owen (2002) added the physical environment factor as a vital component of an ecological model of physical activity. Interventions targeting multiple dimensions of the environment play an important role in enhancing worker physical activity and health as well as business performance (Pronk, 2009; Pronk \& Kottke, 2009). However, progress in this area has been limited by the lack of adequate measures. Only a limited number of measures of workplace environmental support relating specifically to physical activity have been reported (Blunt \& Hallam, 2010; Prodaniuk, Plotnikoff, Spence, \& Wilson, 2004). 
A brief measure focusing on workplace environmental support for only physical activity would be a useful and practical tool for program developers and/or occupational health professionals, but available instruments tend to measure environmental support for multiple health-related behaviors with numerous items. A recently developed instrument focusing on physical activity contains six items that can be easily administered to workers in a few minutes, the Perceived Workplace Environment Scale (PWES) (Prodaniuk et al., 2004). The PWES measures employees' perceptions of their workplace environments that support physical activity and includes six dimensions: individual, social, organizational, community, policy, and physical environment (Prodaniuk et al.).

The purpose of this paper is to describe the translation and evaluation of the psychometric properties of the newly translated Chinese version of the PWES (PWES-C) in a sample of Taiwanese information technology (IT) professionals. The specific aims of this paper are: (a) to describe the translation process of the PWES from English into Chinese; (b) to examine the distribution of the data, internal consistency reliability, and construct validity of the Chinese version.

\section{Background}

In the research literature on workplace health promotion, well-established instruments have been used to assess workplace environments, including the Checklist of Health Promotion Environments at Worksites (CHEW) (Oldenburg, Sallis, Harris, \& Owen, 2002), the Heart Check (Golaszewski \& Fisher, 2002), the Environmental Assessment Tool (EAT) (Parker, DeJoy, Wilson, Bowen, \& Goetzel, 2010), and the Worksite Supportive Environments for Active Living Survey (SEALS) (Blunt \& Hallam, 2010). Except for the Worksite SEALS, these instruments measure environmental 
support for multiple health-related behaviors (ranging from 2 to 5), including physical activity. They are relatively objective measures with many items (ranging from 100 to 226), requiring site visits/observations or interviews with key corporate managers.

Individual items are rated on a dichotomous scale, indicating the presence or absence of environmental support for specific health-related behaviors. In reality, different degrees of environmental support for specific health-related behaviors are possible, and this is not reflected in these instruments. The CHEW focuses mostly on the physical dimension of the environment; the Heart Check focuses on the individual, organizational, policy, and physical environments; and the EAT focuses on the physical and social environments. None of the three instruments attempts to reflect all six dimensions of the ecological model for health promotion. Although these instruments have been shown to have acceptable reliability and/or validity, the observational measures may not be easy to complete and the raters may require substantial training and significant experience to achieve reliable results (Beresford et al., 2010). The 28-item Worksite SEALS (Blunt \& Hallam, 2010) is a reliable and valid self-report measure of perceptions of the environment using a 4-point Likert scale, but is limited to physical and social dimensions of the environment.

Development of the Perceived Workplace Environment Scale. Prodaniuk et al. (2004) constructed a measure of PWES based on their original instrument, the Workplace Physical Activity Assessment Tool (WPAAT) (Plotnikoff, Prodaniuk, Fein, \& Milton, 2005). The WPAAT was developed based on their conceptual framework of an ecological workplace physical activity model (Plotnikoff et al.). This model was based on the work of McLeroy et al. (1988) who proposed an ecological framework for health 
promotion, and Sallis and Owen (2002) who added the physical environment as a potential important component for physical activity.

The ecological workplace physical activity model is composed of six overlapping dimensions of the workplace environment: individual, social, organizational, community, policy, and physical environment (Plotnikoff et al., 2005). Individual refers to factors in the workplace influencing worker characteristics regarding physical activity, such as knowledge, attitude, and skills. Social reflects the influence of the corporate culture, social relationships, peer and supervisor attitudes regarding worker physical activity. Organizational refers to how the organizational structure impacts worker physical activity, such as infrastructure, leadership, and desire to promote physical activity. Community refers to how the workplace interacts or partners with, or uses other organizations, community, or government resources that may promote worker physical activity. Policy is the workplace's policies regarding worker physical activity. Physical environment refers to the workplace's physical environment as it affects worker physical activity, such as buildings, grounds, and surrounding areas (Plotnikoff et al.; Prodaniuk et al., 2004).

The PWES (Prodaniuk et al., 2004) was modified from a 45-item WPAAT "yes/no" assessment tool (Plotnikoff et al., 2005) to a 6-item "5-point Likert scale" survey instrument. Each PWES item measures perceptions concerning one of the six environmental dimensions of the ecological workplace physical activity model. Items are presented in Table 3.1. General instructions direct participants to indicate the extent to which the workplace environment supports physical activity. Responses are quantified on a 5 -point Likert scale: $1=$ none, $2=$ a little, $3=$ some, $4=$ quite a lot, $5=$ a great amount . 
The use of a 5-point rating scale allows an assessment of varying degrees of environmental support for physical activity, and thus has the potential to yield more accurate information.

Scoring. Scores are calculated for the six items by averaging the item responses, with a potential range of one to five. A higher score reflects a more supportive workplace environment for physical activity.

Table 3.1 Items of the Original Perceived Workplace Environment Scale (PWES)

\begin{tabular}{ll}
\hline \multicolumn{1}{c}{ Dimension } & \multicolumn{1}{c}{ Item } \\
\hline Individual & $\begin{array}{l}\text { How much information is provided in your workplace educating } \\
\text { and/or encouraging employees about physical activity? }\end{array}$ \\
Social & $\begin{array}{l}\text { Is there a positive social climate that encourages physical activity in } \\
\text { your workplace? }\end{array}$ \\
Organizational & $\begin{array}{l}\text { How much organizational capacity (i.e. infrastructure, will, and } \\
\text { leadership) is there in your workplace that promotes physical activity } \\
\text { for employees? }\end{array}$ \\
Community & $\begin{array}{l}\text { Has your organization used any services or resources in the } \\
\text { community to support the physical activity of employees? (examples: } \\
\text { local recreation centre, community events) }\end{array}$ \\
Policy & $\begin{array}{l}\text { Does your workplace have policies that promote the physical activity } \\
\text { of employees? (examples: no meetings scheduled over lunch, } \\
\text { subsidized memberships at a fitness centre) }\end{array}$ \\
Physical & $\begin{array}{l}\text { Are there convenient and appropriate facilities that you can access in } \\
\text { order to do physical activity during the workday? }\end{array}$ \\
\hline
\end{tabular}

Previous reliability and validity. Psychometric properties of the original PWES were initially tested in a sample of 897 employees. Evidence for construct validity was provided by confirmatory factor analysis supporting a unidimensional factor structure $(\mathrm{NFI}=.95, \mathrm{IFI}=.95, \mathrm{CFI}=.95, \mathrm{SRMSR}=.06, \mathrm{RMSEA}=.11[90 \% \mathrm{CI}=.09-.13])$. Internal consistency reliability was reported to be acceptable with a Cronbach's $\alpha$ coefficient of .83 and test-retest reliability of $r=.97$ (Prodaniuk et al., 2004). In the 
development stage, item content relevance of the PWES was evaluated by a panel of 15 experts including researchers, workplace physical activity practitioners, and workers (Prodaniuk et al.). In their work, construct validity was also supported by significant ( $p$ $=.01$ ) positive relationships with leisure-time physical activity and workplace physical activity.

\section{Methods}

\section{Design, Settings and Sample}

A cross-sectional descriptive research design was used. The study was approved by the Institutional Review Board at the University of Michigan. Participants were recruited from three IT companies in Northern Taiwan. A systematic sample of 735 Taiwanese IT professionals ( 245 from each of the three companies) was drawn from a list of potential participants. That is, occupational health professionals from the three companies were guided to use a sampling interval of 4 with a random start between 1 and 10 to select 245 out of approximately 1,000 IT professionals from their respective companies. Eligible participants were those who met the inclusion criteria: (a) full-time IT professionals who perform knowledge work in professional positions; (b) aged 18 years or older; (c) no physical limitations or medical problems that would prevent physical activity performance; and (d) not currently pregnant women.

\section{Data Collection}

A total of 735 survey packages were distributed by the IT department assistants from each of the three companies. Each package included an informed consent letter, a self-administered questionnaire (Appendices A and B), a $\$ 3$ gift card, and a return envelope. The letter explained the purpose of the study and invited the IT professional to 
participate in the study. Return of the completed questionnaire represented the respondent's consent to participate in the study. The completed questionnaire was enclosed in a sealed envelope labeled with the principal investigator's name, was returned to the department assistant, and then collected by the investigator. The usable response rate was $78.4 \%$ (576 out of 735). For purposes of this paper, one case was removed from the dataset because one missing value was found in the PWES-C, resulting in a sample size of 575. Details of the study's recruitment methods are further described in Chapter 4.

\section{Instrument Translation}

Permission to begin the translation process was obtained from the author of the PWES (Prodaniuk et al., 2004). The PWES in English was translated into Chinese using a modified committee approach (Behling \& Law, 2000; Geisinger, 1994; Harkness, Pennell, \& Schoua-Glusberg, 2004), with the consideration of cross-cultural equivalence (Flaherty et al., 1988).

Translation process. The committee included two skilled translators, a referee, and the principal investigator; each of them had sufficient cultural and linguistic knowledge. They were all women and from Taiwan. Two bilingual translators and the referee had been in the United States for more than ten years, while the principal investigator was in this country for about four years at the time of the translation. The first translator was an American Translators Association (ATA)-certified translator from English into Chinese and had over ten years experience in translation, writing, editing, and proofreading in a variety of areas, including health care and survey instruments. She earned a bachelor's degree in Foreign Languages and Literature (English major) from 
National Taiwan University and a master's degree in Performance Studies from New York University. The second translator was a member of ATA and a certified PRO member of ProZ.com, the world's largest translator community. She had over ten years experience in translation and editing in many different subject areas, ranging from business surveys and health care to IT and tourism. She received a bachelor's degree in Foreign Languages and Literature (English major) from National Chung-Hsing University, Taiwan and a master's degree in translation from Monterey Institute of International Studies. The bilingual referee was a PhD-prepared nursing professor in the Division of Nursing Business and Health Systems at the University of Michigan who had extensive experience with instrument design and translation issues.

Before translation, brief background information related to the project and source PWES was provided to each committee member to put the translators in the best position to produce a good translation. Two translators independently translated the English language PWES into Chinese and were asked to keep notes of their translation queries, compromises, and problems. In addition, they could clarify with the investigator about any questions they might have during the course of their work.

Following the translations, both Chinese versions of the PWES, along with the agenda for the reconciliation meeting were given to each committee member. Then the committee met in a teleconference to compare and review both versions of the translated PWES item by item to reconcile discrepancies and to produce a consensus version. At the reconciliation meeting chaired by the referee, each translator could explain why certain translation choices were made, and the others could explain why they reacted to the translation as they did. While some very minor differences occurred between the two 
Chinese versions, they were easily resolved by consensus. For example, a minor difference was found in the translation of "local recreation center." One translated this phrase as "local recreation center" and another translated it as "local activity center." Since activity centers are more available than recreation centers in a typical Taiwanese community, the committee reached a consensus that it is therefore more appropriate to use the term "local recreation/activity center" to denote "local recreation center." Through this discursive process, the final Chinese translation reflected the entire committee's best judgment.

Immediately after the reconciliation meeting, the investigator summarized the changes and resubmitted it to the committee for further review. Each committee member reviewed the revised version and achieved a consensus on a final version.

Content equivalence. Content equivalence (Flaherty et al., 1988) was established in two ways. First, the final Chinese version of the PWES (PWES-C) was reviewed for content equivalence by the committee conducting translation as described above. Second, a panel of six Taiwanese experts, who had experience working with IT or white-collar workers, were asked to rate the cultural relevancy and clarity of each item on the PWESC. These six experts were in the areas of occupational health nursing, occupational medicine, health behavior and education, physical activity, and instrument development. Each item was rated on a 4-point Likert scale $(1=$ not relevant; $2=$ somewhat relevant but unable to assess relevance without item revision; 3 = quite relevant but needs minor alteration; $4=$ very relevant and succinct) (Lynn, 1986). The expert panel was asked to provide their suggested revisions for items they scored below 4 . The content validity index (CVI) was computed to quantify the extent of expert agreement based on the 
proportion of items rated as 3 or 4 (quite/very relevant) (Waltz, Strickland, \& Lenz, 2005). The CVI for the entire scale (S-CVI) was 1.0. The CVI for each item (I-CVIs) was 1.0 , except for item $6(\mathrm{CVI}=.83)$, meeting the requirement to be considered content valid (Lynn). One expert suggested item 3 (see Table 3.2) to be separated into three individual items (i.e., infrastructure, will, and leadership). Thus, she further suggested item 6 (see Table 3.2) to be deleted because it articulated a similar concept about "infrastructure." However, the researchers decided to maintain the structure of the original instrument; that is, item 3 was not divided into three items so that item 6 was retained accordingly. Based on the input of the expert panel, one revision was made to item 6 of the PWES-C. Namely, "during or after working hours" was added in the parenthesis after "during the workday" to make it clear to the respondents.

Semantic and technical equivalence. To establish semantic and technical equivalence (Flaherty et al., 1988), the final version of the PWES-C was pretested with 10 Taiwanese IT professionals ( 7 men and 3 women; independent of major study participants) from three IT companies. They were asked to indicate the words, phrases, and sentences that seem strange or unusual in their language; and to identify any problems in completing all items, such as clarity and comprehensibility of the instrument as well as the method of assessment (Carlson, 2000; Flaherty et al.; Wang, Lee, \& Fetzer, 2006). All of the participants responded that the items were easily understood without difficulty in wordings. Only one revision was made based on the participant's suggestions by adding one example (i.e., subsidies for physical activity groups) to item 5 (see Table 3.2) because this benefit is commonly provided by many IT companies in Taiwan. 
After the initial pretesting, the second pretesting of the PWES-C was conducted along with other instruments that were used in the major study at the same time. This time, the entire survey was pretested with a sample of 20 Taiwanese IT professionals (17 men and 3 women) from three IT companies. The purpose was to ensure appropriateness and clarity of item wording, ease of response, as well as correct instructions and format (Brink \& Wood, 1998; Waltz et al., 2005). It took approximately 3 minutes and 30 minutes for respondents to complete the PWES-C and the entire survey, respectively. Following pretesting, an interview was conducted with 10 respondents individually. They were asked to identify any problems they had completing the survey and suggestions for improvements. For the PWES-C, all 10 interviewees confirmed the clarity of item wording. As a result, no modifications to the final version of the PWES-C were made. Actual Chinese version of the PWES-C is presented in Table 3.3. 
Table 3.2 Chinese Version of the Perceived Workplace Environment Scale (PWES-C)

These questions relate to your perceptions about your workplace environment relative to physical activity. Please circle the number that best describes your response to each statement.

\begin{tabular}{|c|c|c|c|c|c|c|}
\hline & & None & A little & Some & $\begin{array}{l}\text { Quite } \\
\text { a lot }\end{array}$ & $\begin{array}{l}\text { A great } \\
\text { amount }\end{array}$ \\
\hline 1. & $\begin{array}{l}\text { How much information is provided in your } \\
\text { workplace educating and/or encouraging } \\
\text { employees about physical activity? }\end{array}$ & 1 & 2 & 3 & 4 & 5 \\
\hline 2. & $\begin{array}{l}\text { Is there a positive social climate that } \\
\text { encourages physical activity in your } \\
\text { workplace? }\end{array}$ & 1 & 2 & 3 & 4 & 5 \\
\hline 3. & $\begin{array}{l}\text { How much organizational capacity (i.e. } \\
\text { infrastructure, will, and leadership) is there in } \\
\text { your workplace that promotes physical } \\
\text { activity for employees? }\end{array}$ & 1 & 2 & 3 & 4 & 5 \\
\hline 4. & $\begin{array}{l}\text { Has your organization used any services or } \\
\text { resources in the community to support the } \\
\text { physical activity of employees? (examples: } \\
\text { local recreation/activity center, community } \\
\text { events) }\end{array}$ & 1 & 2 & 3 & 4 & 5 \\
\hline 5. & $\begin{array}{l}\text { Does your workplace have policies that } \\
\text { promote the physical activity of employees? } \\
\text { (examples: no meetings scheduled over } \\
\text { lunch, subsidies for memberships at a local } \\
\text { fitness center or physical activity groups) }\end{array}$ & 1 & 2 & 3 & 4 & 5 \\
\hline 6. & $\begin{array}{l}\text { Are there convenient and appropriate } \\
\text { facilities that you can access in order to do } \\
\text { physical activity during the workday } \\
\text { (including during or after working hours)? }\end{array}$ & 1 & 2 & 3 & 4 & 5 \\
\hline
\end{tabular}


Table 3.3 知覺職場身體活動環境量表中文版

以下問題是關於您對職場環境有關身體活動方面的認知。對於每一句敘述, 請圈 選一個最能表達您的看法的數字。

\section{無 少許 有些 相當多 很多}

1. 您的工作場所提供了多少資訊以宣導及 $\quad \begin{array}{llllll}1 & 2 & 3 & 4 & 5\end{array}$

（或）鼓勵員工從事身體活動？

2. 您的工作場所是否具有鼓勵從事身體活 $\quad \begin{array}{llllll}1 & 2 & 3 & 4 & 5\end{array}$ 動的正面風氣?

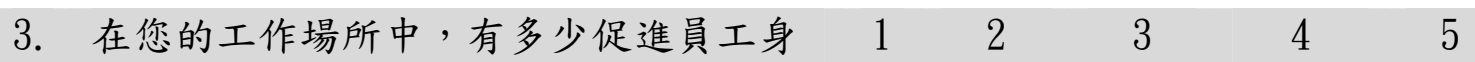
體活動的組織能力（也就是基礎設施、 推動意願和領導力）？

4. 您的組織曾使用社區的任何服務或資 $\begin{array}{lllll}1 & 2 & 3 & 4 & 5\end{array}$ 源，來支持員工的身體活動嗎？（例 如：當地休閒／活動中心、社區活動）

5. 您的工作場所是否訂有鼓勵員工從事身 體活動的政策？（例如：不在午餐時間 開會、補助健身中心會員費或社團活動 費用）

6. 是否有便利及合適的設施, 可以讓您在 $\quad \begin{array}{llllll}1 & 2 & 3 & 4 & 5\end{array}$ 工作日（包括上班或下班時間）用以從 事身體活動? 
Construct validity. Construct validity was examined using confirmatory factor analysis (CFA) to see if the proposed factor structure of the PWES-C was supported in the current study. CFA is based on theory and empirical foundations that allow researchers a priori to specify hypothesized factor structure and then determine how well the proposed measurement model fits the data (Nunnally \& Bernstein, 1994).

Reliability. After a confirmation of the factor structure, reliability was determined by the following criteria. First, internal consistency of the PWES-C was estimated by using Cronbach's alpha coefficient $\geq .70$, and alpha change $\leq .10$ if the item was deleted. Second, a corrected item-total correlation and an inter-item correlation had to be $\geq .30$ (Nunnally \& Bernstein, 1994). Third, the mean of each item had to be close to the center of the range of possible scores (DeVellis, 2003).

Criterion equivalence was not tested because a previously established criterion measure of the same construct was not found in the Chinese language.

Conceptual equivalence. To determine whether the instrument assesses the same theoretical construct in different cultures (Flaherty et al., 1988), conceptual equivalence was established by examining whether the PWES-C (independent variable) had significant relationships with the dependent variables, an overall physical activity score and a leisure-time physical activity score, measured by the International Physical Activity Questionnaire-Taiwan long form. If supportive workplace environments are observed to have a positive relationship with the physical activity level of employees in Taiwanese culture under study as they did in Canadian culture where the PWES was originally developed, the same predicted direction in both the English and Chinese versions of the 
PWES would provide support for the conceptual equivalence of the PWES-C (Flaherty et al.).

\section{Description of the Other Measure}

The Chinese version of the 27-item International Physical Activity QuestionnaireTaiwan (IPAQ-Taiwan) long form (Taiwan Bureau of Health Promotion, 2007) was used as a measure of physical activity. The IPAQ assessed moderate and vigorous intensity physical activity as well as walking in four domains (i.e., transportation, work, domestic/yard work, and leisure-time physical activity) over the last 7 days. Sitting time was evaluated separately by hours of sitting time on a weekday and weekend day. The English version of the IPAQ was translated into Chinese (IPAQ-Taiwan) and certified by the international prevalence study team (Liou, 2004). International validation of the IPAQ has been reported by Craig et al. (2003). Support for the reliability and validity of the IPAQ-Taiwan self-administered long form was provided by Liou in her study conducted with 141 Taiwanese adults aged 18-65. For example, the test-retest reliability using Spearman's rho was .78. Content validity indices were .99 for language equivalence and .99 for meaning similarity between the English and Chinese versions. Criterion validity assessed against RT3 accelerometer data was acceptable (rho $=.41)$.

For purposes of this paper, data were reported as a continuous measure according to guidelines for data processing and analysis of the IPAQ (IPAQ Research Committee, 2005). First, the scores (MET×minutes/week) for walking, moderate (not including walking score) and vigorous intensity physical activity within each domain were computed separately by multiplying the frequency (days/week), duration (minutes/day), and the corresponding metabolic equivalent value (MET; e.g., 3.3 for walking, 4.0 for 
moderate and 8.0 for vigorous physical activity in the work domain). Second, physical activity scores for work, domestic/yard work, and leisure-time physical activity were computed by summing scores across walking, moderate (not including walking score), and vigorous intensity physical activity. The transportation physical activity score was computed by summing scores from walking and cycling. Finally, an overall total physical activity score (MET $\times$ minutes/week) was computed as a sum of total work, transportation, domestic/yard work, and leisure-time physical activity.

\section{Data Analysis}

Prior to data analyses, missing data were examined as an indicator of the data quality, assuming that items were left blank when participants did not understand a given item. Score distributions were examined for the PWES-C. Ceiling and floor effects were assessed by computing the percentage of participants with the highest and lowest possible score for the PWES-C. Internal consistency reliability for the PWES-C was assessed by examining Cronbach's alpha, inter-item correlations, and corrected item-scale correlations.

SPSS 18.0 for Windows was used for descriptive data analyses. Frequencies and descriptive statistics were performed to describe sample characteristics. T-tests were used to determine if there was a statistically significant difference between men and women. Conceptual equivalence of the PWES-C was assessed by examining Pearson correlation coefficients.

Construct validity for the PWES-C was assessed with CFA via structural equation modeling (SEM) method, using the EQS 6.1 program (Bentler, 2006). Multivariate normality was evaluated using Mardia's normalized estimate which assesses the degree 
of kurtosis in the data. Bentler suggests that a value greater than 3 is indicative of nontrivial positive kurtosis; a multivariate normal data has a normalized estimate of 3 . Tests for multivariate kurtosis showed that our Mardia's normalized estimate was 8.82, indicating that the data violate the assumption of multivariate normality. As a result, we employed the Satorra-Bentler (S-B) scaling method to handle non-normal data (Finney \& DiStefano, 2006). All analyses were performed on covariance matrices using maximum likelihood estimation with the S-B scaling method (robust estimation), which adjusts the $\chi^{2}$, fit indices, and standard errors for non-normality (Finney \& DiStefano). To determine whether the hypothesized measurement model fits the data, the goodness of fit statistics were evaluated. We used the S-B $\chi^{2}$ and S-B scaled indices: nonnormed fit index (NNFI), comparative fit index (CFI), Bollen's (IFI) fit index, and root mean-square error of approximation (RMSEA) to evaluate the fit of the models (Hu \& Bentler, 1999; Yu \& Muthén, 2002). For fit indices, a value $\geq .95$ and for the misfit RMSEA index, a value $\leq .06$ indicate adequate fit. However, $\chi^{2}$ is sensitive to sample size, so it is difficult to obtain a nonsignificant $\chi^{2}$ when the sample size is large (Hu \& Bentler; Yu \& Muthén). To compare models with robust estimation, we used calculations based on work by Satorra and Bentler (2001) to correct the $\chi^{2}$ difference test for the S-B scaled $\chi^{2}$. The Wald and Lagrange multiplier tests for dropping and adding parameters, respectively, were examined.

\section{Results}

\section{Descriptive Statistics}

Descriptive statistics from the sample of 575 Taiwanese IT professionals showed that the participants' age ranged from 24 to $60(M=33.7, S D=6.1)$. The sample included 
$466(81 \%)$ men and $109(19 \%)$ women. Table 3.4 presents descriptive statistics and distribution of data for the PWES-C and IPAQ-Taiwan. Scores on the PWES-C indicated somewhat below average levels of support for physical activity in workplace environments. Highest scores were reported for a positive social climate that encourages physical activity in their workplace and the lowest scores were reported for the organization's ability to use community services or resources to support worker physical activity (data not shown). There was no significant gender difference for the PWES-C. A significant gender difference was noted for the IPAQ-Taiwan, suggesting that men were more physically active than women $(t=3.6, p<.001)$. The data were normally distributed as defined by the skew index (an absolute value not exceeding 3.0) (Kline, 2005). Minor potential floor effects were observed for the PWES-C, with $2.6 \%$ reporting the lowest possible scores. Potential ceiling effects were not obvious, with very few participants reporting the highest possible scores for the PWES-C.

Table 3.4 Descriptive Statistics and Distribution of Data for the PWES-C and IPAQTaiwan $(\mathrm{N}=575)$

\begin{tabular}{lcccccc}
\hline \multicolumn{1}{c}{ Scale } & $\begin{array}{c}\text { Men }(\boldsymbol{n}=\mathbf{4 6 6}) \\
\boldsymbol{M}(\boldsymbol{S D})\end{array}$ & $\begin{array}{c}\text { Women }(\boldsymbol{n}=\mathbf{1 0 9}) \\
\boldsymbol{M}(\boldsymbol{S D})\end{array}$ & Range & Skewness & $\begin{array}{c}\text { Ceiling } \\
\text { effect \% }\end{array}$ & $\begin{array}{c}\text { Floor } \\
\text { effect \% }\end{array}$ \\
\hline PWES-C & $2.7(.80)$ & $2.6(.88)$ & $1-5$ & .03 & .3 & 2.6 \\
IPAQ-Taiwan & $2065(2169)$ & $1441(1476)$ & $0-12600$ & 1.96 & .2 & 8.2 \\
\hline
\end{tabular}

Note. PWES-C $=$ Chinese Version of the Perceived Workplace Environment Scale; IPAQ-Taiwan = International Physical Activity Questionnaire-Taiwan.

\section{Confirmatory Factor Analysis}

Three different models of the PWES-C were tested. Table 3.5 summarizes the fit statistics of the three models tested using the robust estimation method. Model 1 hypothesized a one-factor structure in which the PWES-C was conceptualized as a 
unidimensional measure. However, the results did not show an acceptable fit for this model; the RMSEA was greater than .06 .

Next, Model 2 posited a two-factor structure (workplace culture: items 1, 2, 3 and resources for physical activity: items $4,5,6$ ) based on intercorrelations of items in the data. This model performed significantly better than Model 1 , and it fits the data reasonably well, with all the fit indices greater than .95 , except for the RMSEA was greater than .06. Nevertheless, the correlation between workplace culture (factor 1) and resources for physical activity (factor 2) was .84, suggesting a substantial amount of shared variance (a common underlying factor) (Tomas \& Oliver, 1999). Moreover, the Lagrange multiplier test suggested adding three direct effects to improve model fit, from factor 2 to items 1, 2, and 3, respectively. Consequently, the results assessed in Model 2 with two factors may indicate that the PWES-C was a global factor, although these three items (items 1, 2, 3) may tap similar aspects (i.e., workplace culture) of the PWES-C.

To establish a more appropriate measurement model, Model 3 hypothesized a global factor of the PWES-C including correlated errors among error variances of the items 1,2 , and 3 . This final model showed an excellent fit to the data, $\mathrm{S}-\mathrm{B} \chi^{2}(\mathrm{df}=6, N=$ $575)=5.5, p=.48 ; \mathrm{NNFI}=1.0, \mathrm{CFI}=1.0, \mathrm{IFI}=1.0$, and RMSEA $=.00(90 \% \mathrm{CI}: .00-$ $.05)$. The final model was found to fit the data significantly better than any of the nested and simpler models ( $p<.001$ for all comparisons). All of the items loaded strongly and significantly onto an underlying, single factor. All the factor loadings were above .60 (Figure 3.1). Cronbach's alpha for the overall PWES-C was .88. 
Table 3.5 Fit Statistics of the PWES-C from Confirmatory Factor Analysis

\begin{tabular}{lrrrrrrc}
\hline \multicolumn{1}{c}{ Model } & S-B $\chi^{2}$ & df & $\boldsymbol{p}$ & NNFI & CFI & IFI & $\begin{array}{c}\text { RMSEA } \\
\mathbf{( 9 0 \% ~ C I ) ~}\end{array}$ \\
\hline 1 (one-factor) & 80.1 & 9 & .00 & .92 & .95 & .95 & $.12(.09-.14)$ \\
2 (two-factor) & 28.1 & 8 & .00 & .97 & .99 & .99 & $.07(.04-.09)$ \\
3 (one-factor with correlated & 5.5 & 6 & .48 & 1.0 & 1.0 & 1.0 & $.00(.00-.05)$ \\
errors among items 1, 2, 3) & & & & & & & \\
\hline
\end{tabular}

Note. PWES-C $=$ Chinese Version of the Perceived Workplace Environment Scale; S-B $\chi^{2}=$ Satorra-Bentler Scaled $\chi^{2}$; NNFI = nonnormed fit index; CFI = comparative fit index; IFI = Bollen's (IFI) fit index; RMSEA = root mean-square error of approximation; $\mathrm{CI}=$ confidence interval.

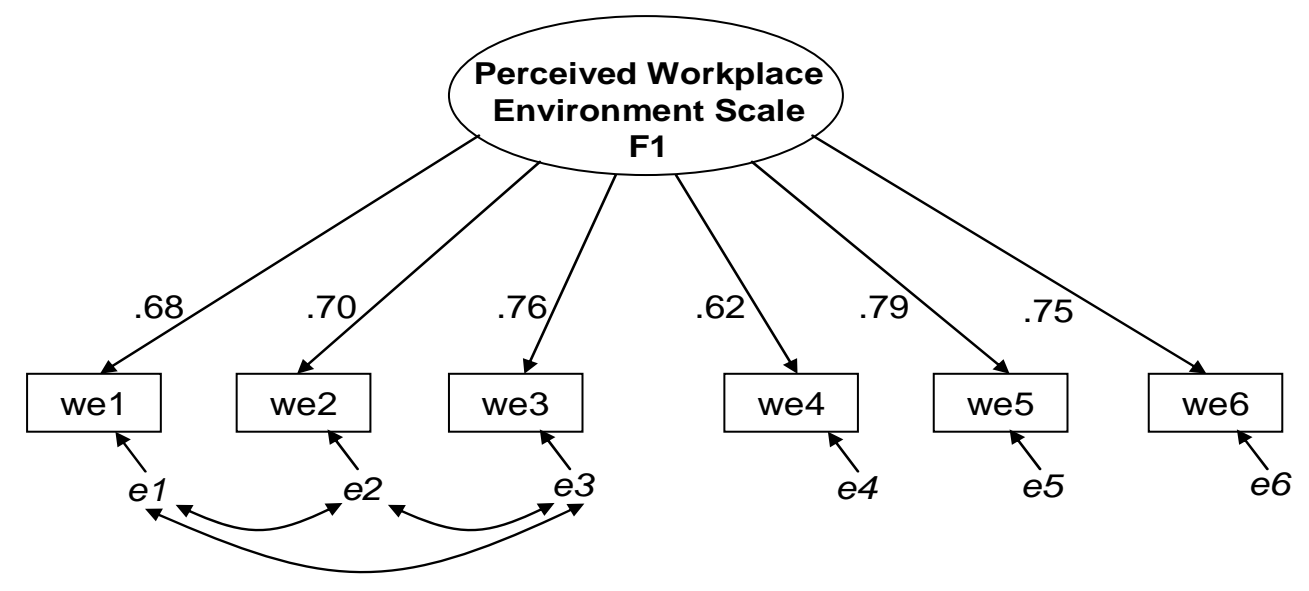

Figure 3.1. Factor structure and standardized factor loadings on the Chinese version of the Perceived Workplace Environment Scale items: Satorra-Bentler Scaled $\chi^{2}(\mathrm{df}=6$, $\mathrm{N}=575)=5.5, p=.48 ; \mathrm{NNFI}=1.0 ; \mathrm{CFI}=1.0 ; \mathrm{IFI}=1.0 ; \mathrm{RMSEA}=.00(90 \% \mathrm{CI}: .00-$ $.05)$. All of the solid line paths are statistically significant at $p<.001$. 


\section{Reliability}

As shown in Table 3.6, internal consistency reliability of the PWES-C was good (Cronbach's $\alpha=.88$ ). The analysis also suggested that the coefficient alpha for the PWES-C would not improve if items were to be deleted. The corrected item-scale correlation of each item in the PWES-C was greater than .30. In general, the higher the corrected correlation between the item and the scale, the better is the item (Nunnally \& Bernstein, 1994). The inter-item correlations of the PWES-C were greater than .30 and less than .80 , indicating that redundancy was less of a concern. Again, the higher the correlations among items, the better are the reliability of individual items (DeVellis, 2003). Finally, the mean of each item (ranging from 2.3 to 2.9) was near the center of the range of possible scores (ranging from 1 to 5) of the PWES-C.

Table 3.6 Internal Consistency Reliability of the PWES-C

\begin{tabular}{ccccc}
\hline Scale & $\begin{array}{c}\text { Cronbach's } \\
\boldsymbol{\alpha}\end{array}$ & $\begin{array}{c}\text { Corrected item- } \\
\text { scale correlations }\end{array}$ & $\begin{array}{c}\text { Inter-item } \\
\text { correlations }\end{array}$ & $\begin{array}{c}\text { Cronbach's } \boldsymbol{\alpha} \text { if } \\
\text { item deleted }\end{array}$ \\
\hline PWES-C & .88 & $.56-.76$ & $.43-.76$ & $.85-.88$ \\
\hline
\end{tabular}

Note. PWES-C $=$ Chinese Version of the Perceived Workplace Environment Scale.

\section{Conceptual Equivalence}

Conceptual equivalence of the PWES-C was supported by significant positive correlations with overall physical activity $(r=.09, p<.05)$ and leisure-time physical activity $(r=.11, p<.01)$, as theoretically expected and empirically observed in Canadian culture. These results add further evidence to the construct validity of the PWES-C. The relationships were weak, though in the expected direction. That is, the higher the 
perceptions of a supportive workplace environment for physical activity, the greater the reported engagement in overall physical activity and leisure-time physical activity.

\section{Discussion}

The English version of the PWES was translated into Chinese and validated for cross-cultural equivalence. The results of this study provide strong support for the reliability and validity of the PWES-C when used in a sample of Taiwanese IT professionals. The instrument behaved in a manner consistent with theoretical expectations. Problems with missing data and potential ceiling and floor effects were very minor.

Sample characteristics of the present study were quite different from those of Prodaniuk et al. (2004) who developed the PWES. We recruited our participants from three IT companies, while Prodaniuk et al. recruited their sample from three organizations (a health authority, an educational institution, and a large urban center). Our sample size $(N=575)$ was relatively smaller than that of Prodaniuk et al. $(N=897)$. Our sample included many more men than women ( $81 \%$ vs. $19 \%)$, whereas the sample of Prodaniuk et al. included more women than men (74\% vs. $26 \%)$ participated in their study. The mean age in our sample was 33.7 compared to 42.2 years in Prodaniuk et al.'s.

The PWES-C demonstrated very few problems with ceiling and floor effects. This indicates that the instrument would be appropriate for assessing a wide range of workplace environments, including very supportive and not supportive workplace environments.

Results supported the reliability of the PWES-C. Internal consistency, corrected item-scale correlations, and inter-item correlations were acceptable for the PWES-C. 
Cronbach's alpha coefficient in our study was higher than that reported by Prodaniuk et al. (2004). Test-retest reliability was not examined in this study; this could be addressed in future research to establish the stability of this Chinese version instrument.

The results of this study provide evidence for the conceptual equivalence and construct validity of the PWES-C. From a theoretical perspective, the PWES-C was positively related to measures of overall physical activity and leisure-time physical activity. The findings were similar to the work of Prodaniuk et al. (2004) who reported weak relationships between the English version of the PWES and workplace physical activity and leisure-time physical activity. The authors noted that the weak relationships may be due to the fact that several factors influence worker physical activity, in addition to workplace environments. Moreover, the degree of environmental supports for physical activity may be another factor accounting for the weak relationships. For instance, a neutral environment may have no or little impact on worker physical activity (Prodaniuk et al.). Additionally, because the data were collected from only three sites, one would not expect a strong relationship between workplace environments and physical activity due to the lack of variance on the perceived workplace environment.

The results of this study identified a single factor structure for the PWES-C underlying responses to the items. However, the inclusion of three correlated errors is necessary to achieve a better model fit. Correlated errors were associated with a common element, the workplace culture that was something only common to items 1 to 3 . The findings were somewhat different from those reported by Prodaniuk et al. (2004) who indicated a unidimensional factor structure for the English version of the PWES. Although fit indices reported in their study were acceptable, their misfit RMSEA index 
was greater than .06 , which were similar but somewhat better than our results for the unidimensional measure $($ RMSEA $=.11$ vs. .12). Nevertheless, both studies suggested that all six items measured one global PWES factor. This was probably because of the general nature of the items, and the overlapping nature of multiple dimensions of the ecological workplace physical activity model.

The PWES-C makes a unique contribution to the existing measures of workplace physical activity environments. It is suitable for large scale studies because it is short. It has the potential to monitor changes over time and predict behavior change. It is consistent with contemporary views on the multidimensional nature of health promotion in the workplace context because it reflects six dimensions of an ecological workplace physical activity model. In terms of its appropriateness in reflecting the environmental support for physical activity, a subjective measure may be more appropriate than an objective measure, because if workplace environments are not perceived as supportive, workers are less likely to engage in physical activity.

The PWES-C is the initial Chinese version of the PWES and a promising instrument for measuring perceived workplace environments. The results of this study provide strong evidence for its reliability and validity when used in a sample of Taiwanese IT professionals. Each dimension of the workplace environment is measured by a single item which may not cover the full scope of experience operating in workplace environments that support physical activity. However, the PWES-C has the potential to be a useful and practical tool for employers, program developers, and occupational health professionals, because it is brief and easy to complete. 


\section{References}

Behling, O., \& Law, K. S. (2000). Translating questionnaires and other research instruments: Problems and solutions. Thousand Oaks, Calif.: Sage Publications.

Bentler, P. M. (2006). EQS 6 structural equations program manual. Encino, CA: Multivariate Software, Inc.

Bentler, P. M., \& Chou, C. (1987). Practical issues in structural modeling. Sociological Methods \& Research, 16(1), 78-117.

Beresford, S. A., Bishop, S. K., Brunner, N. L., Duncan, G. E., McGregor, B. A., McLerran, D. F., et al. (2010). Environmental assessment at worksites after a multilevel intervention to promote activity and changes in eating: The PACE project. Journal of Occupational and Environmental Medicine, 52 Suppl 1, S2228.

Blunt, G. H., \& Hallam, J. S. (2010). The worksite supportive environments for active living survey: Development and psychometric properties. American Journal of Health Promotion, 25(1), 48-57.

Brink, P. J., \& Wood, M. J. (1998). Advanced design in nursing research (2nd ed.). Thousand Oaks, Calif.: Sage Publications.

Carlson, E. D. (2000). A case study in translation methodology using the healthpromotion lifestyle profile II. Public Health Nursing, 17, 61-70.

Craig, C.L., Marshall, A.L., Sjostrom, M., Bauman, A. E., Booth, M. L., \& Ainsworth, B. E., et al. (2003). International physical activity questionnaire: 12-country reliability and validity. Medicine and Science in Sports and Exercise, 35(8), 13811395.

DeVellis, R. F. (2003). Scale development: Theory and applications. Thousand Oaks, Calif:: Sage Publications, Inc.

Finney, S. J., \& DiStefano, C. (2006). Non-normal and categorical data in structural equation modeling. In G. R. Hancock, \& R. O. Mueller (Eds.), Structural equation modeling: A second course. Greenwich, CT: IAP.

Flaherty J. A., Gavira, F. M., Pathak, D., Mitchell, T., Wintrob, R., Richman, J. A. et al. (1988). Developing instruments for cross-cultural psychiatric research. Journal of Nervous and Mental Disease, 176(5), 257-263.

Geisinger, K. F. (1994). Cross-cultural normative assessment: Translation and adaptation issues influencing the normative interpretation of assessment instruments.

Psychological Assessment, 6(4), 304-312. 
Golaszewski, T., Allen, J., \& Edington, D. (2008). Working together to create supportive environments in worksite health promotion. American Journal of Health Promotion, 22(4), 1-10, iii.

Golaszewski, T., \& Fisher, B. (2002). Heart check: The development and evolution of an organizational heart health assessment. American Journal of Health Promotion, $17(2), 132-153$.

Harkness, J., Pennell, B.-E., \& Schoua-Glusberg, A. (2004). Survey questionnaire translation and assessment. In S. Presser., J. M. Rothgeb, M. P. Couper, J. T. Lessler, E. Martin, J. Martin, \& E. Singer (Eds.), Methods for Testing and Evaluating Survey Questionnaires (pp. 453-473). New York: John Wiley \& Sons, Inc.

Hu, L.-t., \& Bentler, P. M. (1999). Cutoff criteria for fit indexes in covariance structure analysis: Conventional criteria versus new alternatives. Structural Equation Modeling: A Multidisciplinary Journal, 6(1), 1 - 55.

IPAQ Research Committee. (2005, November). Guidelines for data processing and analysis of the international physical activity questionnaire (IPAQ). Retrieved June 20, 2009, from http://www.ipaq.ki.se/scoring.pdf

Kline, R. B. (2005). Principles and practice of structural equation modeling. New York: Guilford Press.

Liou, Y. M. (2004). Development and verification of validity and reliability of the Taiwan version of international physical activity questionnaires. Unpublished doctoral dissertation, National Taiwan University, Taipei. (in Chinese)

Lynn, M. R. (1986). Determination and quantification of content validity. Nursing Research, 35(6), 382-385.

McLeroy, K. R., Bibeau, D., Steckler, A., \& Glanz, K. (1988). An ecological perspective on health promotion programs. Health Education Quarterly, 15(4), 351-377.

Nunnally, J. C., \& Bernstein, I. H. (1994). Psychometric Theory. New York: McGrawHill.

Oldenburg, B., Sallis, J. F., Harris, D., \& Owen, N. (2002). Checklist of health promotion environments at worksites (CHEW): Development and measurement characteristics. American Journal of Health Promotion, 16(5), 288-299.

Parker, K. B., DeJoy, D. M., Wilson, M. G., Bowen, H. M., \& Goetzel, R. Z. (2010). Application of the Environmental Assessment Tool (EAT) as a process measure for a worksite weight management intervention. Journal of Occupational and Environmental Medicine, 52 Suppl 1, S42-51. 
Plotnikoff, R. C., Prodaniuk, T. R., Fein, A. J., \& Milton, L. (2005). Development of an ecological assessment tool for a workplace physical activity program standard. Health Promotion Practice, 6(4), 453-463.

Prodaniuk, T. R., Plotnikoff, R. C., Spence, J. C., \& Wilson, P. M. (2004). The influence of self-efficacy and outcome expectations on the relationship between perceived environment and physical activity in the workplace. International Journal of Behavioral Nutrition and Physical Activity, 1(1), 7.

Pronk, N. P. (2009). Physical activity promotion in business and industry: evidence, context, and recommendations for a national plan. Journal of Physical Activity \& Health, 6 Suppl 2, S220-235.

Pronk, N. P., \& Kottke, T. E. (2009). Physical activity promotion as a strategic corporate priority to improve worker health and business performance. Preventive Medicine, 49(4), 316-321.

Sallis, J. F., \& Owen, N. (2002). Ecological models of health behavior. In K. Glanz, F. M. Lewis, \& B. K. Rimer (Eds.), Health behavior and health education: Theory, research, and practice (3rd ed., pp. 462-484). New York: John Wiley \& Sons.

Satorra, A., \& Bentler, P. M. (2001). A scaled difference chi-square test statistic for the moment structure analysis. Psychometrika, 66(4), 507-514.

Taiwan Bureau of Health Promotion, Department of Health. (2007). International physical activity questionnaire Taiwan version: Long form. Retrieved June 20, 2009, from http://www.bhp.doh.gov.tw/BHPnet/Portal/Them_Show.aspx? Subject $=200712250028 \&$ Class $=2 \& \mathrm{No}=200903250002$ (in Chinese)

Tomas, J. M., \& Oliver, A. (1999). Rosenberg's self-esteem scale: Two factors or method effects. Structural Equation Modeling: A Multidisciplinary Journal, 6(1), 84 - 98.

van Poppel, M. N. M., \& Engbers, L. H. (2009). Programs designed to improve employee health through changes in the built environment. In N. P. Pronk (Ed.), ACSM's worksite health handbook: A guide to building healthy and productive companies (2nd ed.). Champaign, IL: Human Kinetics.

Waltz, C. F., Strickland, O. L., \& Lenz, E. R. (2005). Measurement in nursing and health research (3rd ed.). New York: Springer Pub.

Wang, W. L., Lee, H. L., \& Fetzer, S. (2006). Challenges and strategies of instrument translation. Western Journal of Nursing Research, 28(3), 310-321.

World Health Organization. (2002). The world health report 2002: Reducing risks, promoting healthy life. Retrieved March 18, 2008, from http://www.who.int/whr/2002/en/whr02_en.pdf 
Yu, C., \& Muthén, B. (2002, April). Evaluation of the model fit indices for latent variable models with categorical and continuous outcomes. Paper presented at the annual meeting of the American Educational Research Association, New Orleans, LA. 


\title{
CHAPTER 4
}

\section{Work Environment and Psychosocial Factors Affecting Physical Activity among Taiwanese Information Technology Professionals}

\begin{abstract}
Information technology (IT) professionals are typically sedentary and little is known about factors that influence their physical activity. This research was guided by a theoretically-supported model using Social Cognitive Theory, incorporating variables from the demand/control model. PURPOSE: To identify determinants of physical activity in IT professionals by examining the relationships among work environment (i.e., supportive workplace environments and job strain), psychosocial factors (i.e., outcome expectations and self-efficacy for physical activity), and physical activity. METHODS: This was a cross-sectional survey of 576 IT professionals from three IT companies in Taiwan. Subjects were 467 men and 109 women with a mean age of $33.7(S D=6.08)$. Subjects completed a Chinese questionnaire that included the International Physical Activity Questionnaire-Taiwan long form, Perceived Workplace Environment Scale, Psychological Job Demands and Job Control Scale, Scale of Exercise Self-Efficacy, Positive and Negative Outcome Expectations Scale, Historical Physical Activity Questionnaire, and background information. Structural equation modeling (SEM) was used to test the model. RESULTS: SEM analyses showed that (a) higher physical activity levels were associated with higher scores on supportive workplace environments, positive outcome expectations, self-efficacy, perceived health, and historical physical
\end{abstract}


activity, as well as shorter work hours and being male; (b) self-efficacy partially mediated the effects of supportive workplace environments on physical activity, while outcome expectations partially mediated the effects of self-efficacy on physical activity; (c) job strain had a significant indirect effect on physical activity through self-efficacy; (d) the final model provided a good fit to the data and accounted for $31 \%$ of the variance in physical activity; and (e) the effect of gender on our findings was not substantial in multigroup analyses. CONCLUSIONS: Work environment and psychosocial factors are both important. This suggests that workplace physical activity interventions directed toward individuals' self-efficacy and outcome expectations in the context of supportive environments may be useful. Further research with a larger number of corporations using a longitudinal design is needed to fully explore the effects of workplace environments. 


\section{Introduction}

The general benefits of physical activity and costs of physical inactivity have been widely documented. Regular physical activity has been associated with many health benefits (e.g., decreased risk for obesity, heart disease, hypertension, diabetes, certain cancers, and depression), while physical inactivity has been associated with numerous chronic diseases and premature death (U.S. Department of Health and Human Services [USDHHS], 2000, 2008). In the context of the workplace setting, studies have demonstrated positive associations between physical activity and better job performance (Pronk et al., 2004) and lower health care costs (Wang, McDonald, Champagne, \& Edington, 2004). Moreover, the benefits of onsite fitness centers/programs for both workers and employers have been well documented, including increased worker productivity, reduced absenteeism and medical costs, improved worker health, decreased industrial injuries, improved corporate image, and less employee turnover (Baun, Bernacki, \& Tsai, 1986; Burton, McCalister, Chen, \& Edington, 2005; Lynch, Golaszewski, Clearie, Snow, \& Vickery, 1990; Musich, Adams, Broder, \& Edington, 1999; Shephard, 1992, 1999). On the contrary, sedentary work behaviors have been documented to cause significant negative health effects. For example, workers whose jobs require long hours of sitting have been shown to have two times the increased risk of cardiovascular disease than those whose jobs required physical effort (Hamilton, Healy, Dunstan, Zderic, \& Owen, 2008).

Despite the benefits of physical activity, however, nearly $40 \%$ of Taiwanese adults did not achieve the recommended amount of physical activity (e.g., moderate physical activity for 30 minutes a day, 5 days a week, or vigorous physical activity for 20 
minutes a day, 3 days a week; see Haskell et al., 2007). In particular, the 25-44 age group and white-collar workers were the least active (Chen et al., 2008). Further, a recent Internet survey showed that Taiwanese information technology (IT) workers were the least active working population, with approximately $70 \%$ considering themselves to be physically inactive (Taiwan 1111 Job Bank, 2007). A study of 106 Taiwanese IT workers revealed that only $4 \%$ engaged in leisure time physical activity (LTPA) $\geq 3$ times/week (Hsu \& Huang, 2001), compared to $30 \%$ of all Taiwanese adults (Ku, Fox, McKenna, \& Peng, 2006).

Since 1990 the IT service business has become one of Taiwan's most vital industries. The employment of IT professionals increased from 65,499 in 2006 to 80,748 in 2009, and is expected to grow steadily (Taiwan Science and Technology Advisory Group, 2007). As a result of global market competition, rapid technological advances, and tight schedules, the IT profession is considered a highly stressful occupation with long work hours, which has contributed to a sedentary lifestyle (Taiwan Council of Labor Affairs [TCLA], 2007). As stated in the World Health Report 2002, increases in inadequate physical activity levels and prolonged sitting are results of changes to living and work patterns (World Health Organization [WHO], 2002). In response, the WHO (2008) emphasized that workplace health promotion programs targeting physical activity and diet are an effective means to prevent chronic diseases and promote health because of their potential to reach many workers and through multiple levels of influence.

Social Cognitive Theory. Physical activity behavior is influenced by a complex, interrelated set of determinants, including personal and environmental factors (Brug, Oenema, \& Ferreira, 2005; Kremers et al., 2006; Spence \& Lee, 2003). The Social 
Cognitive Theory (Bandura, 1986) thus is best suited for understanding the influence of personal (psychosocial, specifically outcome expectations and self-efficacy) and environmental (work environment, specifically workplace physical activity environments and job strain) factors on physical activity among workers.

Psychosocial factors. Self-efficacy is defined as a person's confidence in their ability to perform a specific behavior necessary to achieve an expected outcome in a particular situation (Bandura, 1986, 1997). People have little motivation to perform a behavior when facing difficulties unless they believe they can produce the desired outcomes by their actions (Bandura, 2004). Self-efficacy has been the most widely investigated variable in worker physical activity studies, and has had a consistent positive correlation with physical activity (Chen \& Chang, 2004; Kaewthummanukul, Brown, Weaver, \& Thomas, 2006; Kao, Lu, \& Huang, 2002; Lee, Huang, \& Kao, 2005; Nishida, Suzuki, Wang, \& Kira, 2004; Payne, Jones, \& Harris, 2002; Prodaniuk, Plotnikoff, Spence, \& Wilson, 2004; Purath, 2006; Sassen, Kok, Schaalma, Kiers, \& Vanhees, 2010; Tavares, Plotnikoff, \& Loucaides, 2009). In addition, self-efficacy served as a mediator in the relationship between perceived workplace environment and workplace physical activity, when used in a combined sample of healthcare, education, and city workers (Plotnikoff, Pickering, Flaman, \& Spence, 2010; Prodaniuk et al.).

Health behaviors are also affected by the outcomes that people expect their performances to produce (Bandura, 2004). Outcome expectations are motivational beliefs about the expected benefits and costs for performing a behavior in particular situations (Bandura; Baranowski, Perry, \& Parcel, 2002). The terms outcome expectations and outcome expectancies have been used interchangeably. Williams, Anderson, and Winett 
(2005) demonstrated that outcome expectancy is labeled and conceptualized in various ways within different theories, but all applications include expected outcomes of a behavior. Surprisingly, very few studies (Prodaniuk et al., 2004; Steinhardt \& Dishman, 1989) have examined the outcome expectations of physical activity in workers, and even fewer (Prodaniuk et al.) have examined outcome expectations in conjunction with selfefficacy for physical activity. Much less attention has been paid to understanding the role of outcome expectations in explaining physical activity of workers as compared to selfefficacy.

According to Williams et al. (2005), perceived benefits are the same as positive outcome expectations, perceived barriers are a subtype of negative outcome expectations, positive outcome expectations are part of the decisional balance pros, and negative outcome expectations are part of the decisional balance cons. In studies of worker physical activity, most found that perceived benefits or pros of physical activity had small, positive associations with physical activity (Kaewthummanukul et al., 2006; Nishida et al., 2004; Prodaniuk et al., 2004; Purath, 2006; Steinhardt \& Dishman, 1989; Tavares et al., 2009). Perceived barriers or cons of physical activity were more consistently and negatively correlated with physical activity (Chen \& Chang, 2004; Kaewthummanukul et al.; Kao et al., 2002; Nishida et al.; Prodaniuk et al.; Purath; Sassen et al., 2010; Steinhardt \& Dishman; Tavares et al.).

Organizational work environment factors. Perceived workplace environments were weakly, though significantly, associated with LTPA and workplace physical activity (Prodaniuk et al., 2004). Subsidies for health club use (organizational policy) were positively related to both LTPA and work-break physical activity. Having access to on- 
site exercise facilities was positively related to LTPA, whereas paid time for non-workrelated physical activity and a safe place to walk outside work were positively related to work-break physical activity (Lucove, Huston, \& Evenson, 2007). Workers with accessible exercise facilities and equipment, stairways, and personal services at worksites were more likely to meet physical activity recommendations than those without these environments. Also, the number of available worksite policies or environments was associated with the likelihood of meeting physical activity recommendations (Dodson, Lovegreen, Elliott, Haire-Joshu, \& Brownson, 2008). Furthermore, qualitative data suggested that a positive social climate for workplace physical activity or incorporating physical activity into the corporate culture may motivate workers to engage in physical activity or be less sedentary at work (Chang, 2007; Tavares \& Plotnikoff, 2008). Yet little research has examined the impact of multiple dimensions of workplace environments on worker physical activity (Prodaniuk et al.).

Individual work environment factors. Karasek's (1979) demand/control model (also named "job strain model") has been tested in various research areas, including health behaviors (Karasek et al., 1998). The model posits that psychological strain results from the combined effects of psychological job demands and the degree of decisionmaking freedom available to workers when facing those demands. In light of the model, low control combined with high demands represents a "high strain job," which may lead to psychological strain and physiological health outcomes, whereas high control combined with low demands represents a "low strain job," which leads to relatively less strain. High control combined with high demands represents an "active job," indicating 
good strain, while low control combined with low demands represents a "passive job," which may lead to negative job learning (Karasek et al., 1998).

The relationships among job demands, job control, job strain (ratio of demands to control), and physical activity from previous research were somewhat inconsistent and varied between sexes. While women with active jobs were more likely to be physically active (Hellerstedt \& Jeffery, 1997), men and older workers with active jobs demonstrated an opposite effect (Kouvonen et al., 2005). Women with low strain jobs were less likely to be active, whereas men with low strain jobs were more likely to be active (Hellerstedt \& Jeffery). Nonetheless, passive jobs, high strain jobs, and low job control had a relatively consistent relationship with lower levels of physical activity or exercise in both men and women (Brisson, Larocque, Moisan, Vezina, \& Dagenais, 2000; Gimeno et al., 2009; Hellerstedt \& Jeffery; Kouvonen et al.; Lallukka et al., 2008). In contrast, some studies did not find a direct relationship between exercise or sedentary behavior and job demands, job control, or job strain (Kaewthummanukul et al., 2006; Landsbergis et al., 1998; Payne et al., 2002, 2005).

In summary, no studies have explored the specific nature of physical activity and its determinants among IT workers. While most worker physical activity studies have examined psychosocial and individual work environment factors, few have focused on broader organizational work environment factors. No published studies have examined the potential influence of work environments on physical activity in Taiwanese IT workers.

The purpose of this study was to identify determinants of physical activity in Taiwanese IT professionals by examining the relationships among work environment (i.e., 
supportive workplace environments and job strain), psychosocial factors (i.e., outcome expectations and self-efficacy for physical activity), and physical activity. Examination of these relationships was guided by a hypothesized model (Figure 4.1) using Social Cognitive Theory (i.e., supportive workplace environments, outcome expectations and self-efficacy for physical activity) (Bandura, 1986), incorporating variables from the demand/control model (i.e., job strain; the ratio of demands to control) (Karasek, 1979). This approach aligns with the view that "Synergy may be achieved by taking the most promising concepts from each model and integrating them for use with specific populations" (Baranowski, Cullen, Nicklas, Thompson, \& Baranowski, 2003, p. 23S). It also addresses the recommendation to examine how environmental and psychosocial variables interact in their effects on physical activity (Marcus, Williams, et al., 2006). In the model, physical activity behavior was posited to be the outcome of a simultaneous influence of conscious and unconscious processes (Kremers et al., 2006). Supportive workplace environments were hypothesized to affect physical activity both directly and indirectly through the mediating role of outcome expectations and self-efficacy for physical activity. The indirect effect reflects the relatively conscious influence of supportive workplace environments on physical activity. The direct effect reflects a relatively automatic or unconscious influence of supportive workplace environments on physical activity. Job strain was hypothesized to affect physical activity directly.

Four key hypotheses that form the model (Figure 4.1) illustrating the direct and indirect paths of influence of the constructs on physical activity are stated below. Seven covariates consist of seniority, perceived health, work hours, historical physical activity, gender, site $\mathrm{B}$, and site $\mathrm{C}$ (with site $\mathrm{A}$ as the reference category). 
Hypothesis 1: Supportive workplace environments will have a significant positive effect on physical activity, outcome expectations, and self-efficacy for physical activity in a model that includes seven covariates.

Hypothesis 2: Job strain (ratio of demands to control) will have a significant negative effect on physical activity in a model that includes seven covariates.

Hypothesis 3: Outcome expectations and self-efficacy for physical activity will have a significant positive effect on physical activity in a model that includes seven covariates.

Hypothesis 4: Outcome expectations and self-efficacy for physical activity will significantly mediate the effects of supportive workplace environments on physical activity in a model that includes seven covariates.

\section{Environmental Influences (Work Environment)}

\section{Personal Factors \\ (Psychosocial)}

\section{Behavioral \\ Outcome}

\section{Covariates}

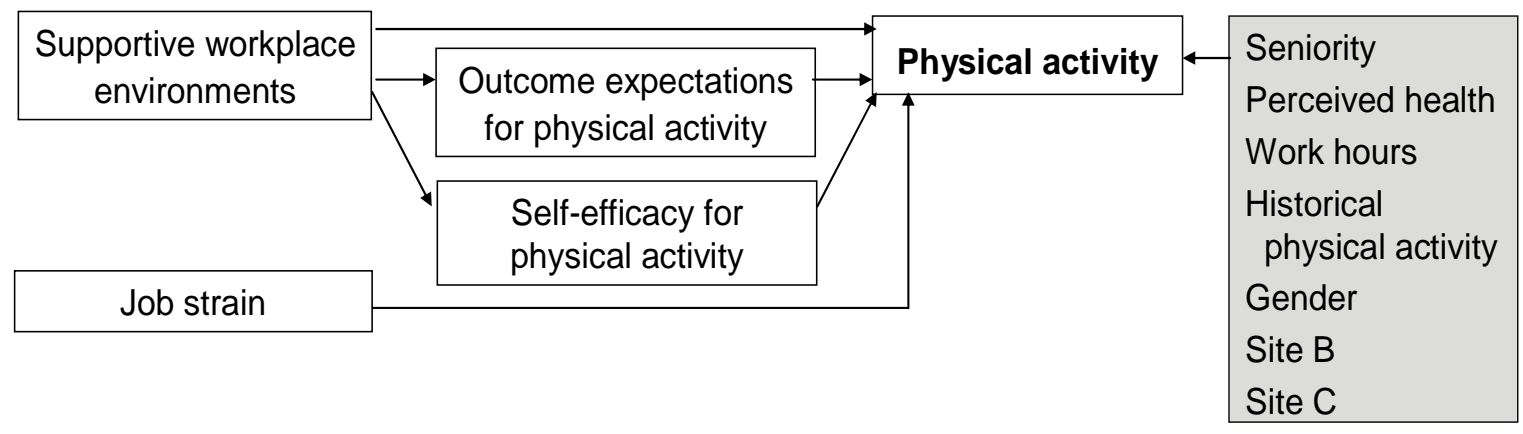

Figure 4.1. Hypothesized workplace physical activity model. 


\section{Methods}

\section{Design, Setting and Sample}

A cross-sectional survey was conducted between March and April 2010. The study was approved by the Institutional Review Board at the University of Michigan. Participants were recruited from three IT companies (identified as A, B, and C) located in northern Taiwan. Compared to sites B and C, site A had relatively few workplace facilities and equipment available for physical activity, such as a walking trail on grounds and a table tennis room. Site B had an aerobics room and an open workout area for physical activity that included three treadmills, three stationary bikes, and one billiard table. In contrast, Site $\mathrm{C}$ had an on-site gym, small-scale fitness centers, a multifunctional sports court, an indoor pool, workout rooms, showers/change rooms, and lockers.

Additionally, all three sites provided clean and safe stairwells with signs encouraging use of stairs. Each company also offered subsidies for physical activity groups and provided employees with knowledge about the benefits of physical activity and tips on getting active. In particular, site B displayed the motivational or informative signage on the stairs that encouraged physical activity and offered relatively more physical activity groups and aerobics classes.

A systematic sample of 735 Taiwanese IT professionals (245 from each of the three companies) was drawn from a list of potential respondents provided by the IT departments of the companies. Specifically, three occupational health professionals (two nurses and one senior manager of Safety and Health Department) from the three companies were guided to use a sampling interval of 4 with a random start between 1 and 
10 to select 245 out of approximately 1,000 IT professionals from their respective companies.

Eligible respondents were those who met the inclusion criteria: (a) full-time IT professionals who perform knowledge work in professional positions (e.g., software engineer, system analyst/engineer, programmer, research and development engineer, process engineer, equipment engineer, sales engineer, etc.); (b) aged 18 years or older; (c) no physical limitations or medical problems that would prevent physical activity performance; and (d) not currently pregnant women. (Note: pregnant women were excluded to avoid the potential confounding influence of this factor.) The first criterion was initially screened by the IT department staff, and eligibility was verified by the respondents' report on their job titles and job type. Three confirmation questions regarding the last three criteria were placed on the cover page of the questionnaire to exclude ineligible participants. These resulted in the exclusion of $67(9.8 \%)$ respondents from the study.

A total of 735 survey packages were distributed by the IT department assistants from each of the three companies. Each package included an informed consent letter, a self-administered questionnaire (Appendices A and B), a \$3 gift card, and a return envelope. The letter explained the purpose of the study and invited the IT professional to participate in the study. It also explained that the questionnaire was expected to take about 25 to 35 minutes to complete. The inclusion of a token financial incentive (a $\$ 3$ gift card) was intended to demonstrate appreciation for the participant's help and to maximize response rates (Dillman, Smyth, \& Christian, 2009). Return of the completed questionnaire represented the respondent's consent to participate in the study. The 
completed questionnaire was enclosed in a sealed envelope labeled with the principal investigator's name, was returned to the department assistant, and then collected by the investigator. A total of two reminder e-mails (two and four weeks after the initial delivery, respectively) were sent by company staff to all participants to thank those who responded and to encourage potential respondents to complete and return the questionnaires (Dillman et al.). The list with participant names was destroyed after the second reminder e-mail was sent.

Response rate. From 735 questionnaires distributed, 687 (93.5\%) were returned from the three IT companies $(89.8 \%, 91.4 \%$, and $99.2 \%$, respectively) within one and half months. Of the 687 respondents, 620 (90.2\%) met the eligibility criteria described above. Forty-four $(6.4 \%)$ cases were disqualified because of missing data $(n=41)$ or overreporting $(n=3)$ on the International Physical Activity Questionnaire-Taiwan (Taiwan Bureau of Health Promotion [TBOHP], 2007), leaving a final response rate of $78.4 \%(N=576)$. There was no statistically significant difference between disqualified $(n$ $=44)$ and qualified $(n=576)$ groups in regard to their demographic data, such as age, gender, marital status, education, job title, seniority, and work hours.

\section{Measures}

All of the constructs in this study were assessed with multi-item indices. The Cronbach's $\alpha$ coefficients of our measures ranged from .78 to .93. Three instrumentsthe Perceived Workplace Environment Scale (Prodaniuk et al., 2004), the Positive and Negative Outcome Expectations Scale (Rovniak, Anderson, Winett, \& Stephens, 2002), and the Historical Physical Activity Questionnaire (Norman, Bellocco, Bergstrom, \& Wolk, 2001; Orsini, Bellocco, Bottai, Pagano, \& Wolk, 2007) originally written in 
English were translated into Chinese using a modified committee approach (Harkness, Pennell, \& Schoua-Glusberg, 2004), with the consideration of cross-cultural equivalence (Flaherty et al., 1988). Further details about the translation process are provided in Chapter 3. Table 4.1 presents the descriptive statistics and internal consistency reliabilities (Cronbach's $\alpha$ ) for the scales. Factor loadings and error for the measured variables (indicators) of latent factors used in the confirmatory factor analysis (CFA) of the measurement model are shown in Table 4.2.

Outcome variable. Physical activity was measured with the 27 -item International Physical Activity Questionnaire-Taiwan (IPAQ-Taiwan) long form (TBOHP, 2007). The IPAQ quantified moderate and vigorous intensity physical activity as well as walking in four domains (transportation, work, domestic/yard work, and LTPA) over the last 7 days. Sitting time was evaluated separately by hours of sitting time on a weekday and weekend day. The English version of the IPAQ was translated into Chinese (IPAQ-Taiwan) and certified by the international prevalence study team (Liou, 2004). In the IPAQ-Taiwan, numerous additional examples of common physical activity performed by Taiwanese adults were added to the original IPAQ to help respondents understand the classification of physical activity intensity and thus answer correctly (Liou).

International validation of the IPAQ was reported by Craig et al. (2003). Support for the reliability and validity of the IPAQ-Taiwan self-administered long form (LS) was provided by Liou (2004) in her study. The study was conducted with a community sample of 141 Taiwanese adults aged 18-65 using systematic and household sampling stratified by age, gender, education, and physical activity level (Liou). For example, the test-retest reliability using Spearman's rho was .78 . Content validity indices were .99 for 
language equivalence and .99 for meaning similarity between the English and Chinese versions. Concurrent validity was established by comparing the long and short selfadministered form of the IPAQ-Taiwan $($ rho $=.86)$. Criterion validity assessed against RT3 accelerometer data was acceptable $($ rho $=.41)$. Evidence of discriminant validity showed that IPAQ-Taiwan LS (moderate physical activity scores: $\geq 150$ vs. $<150$ minutes/week) has the ability to discriminate between people with varying leg muscle strength $(p<.05)$, waist to hip ratio $(p=.01)$, glycosylated hemoglobin $(\mathrm{HbA1C})(p$ $<.05)$, total cholesterol $(p<.01)$, and high-density lipoprotein $(p<.05)$ (Liou).

For analysis purposes, data were reported as a continuous measure according to guidelines for data processing and analysis of the IPAQ (IPAQ Research Committee, 2005). First, the scores (MET $\times$ minutes/week) for walking, moderate (not including walking score) and vigorous intensity physical activity within each domain were computed separately by the multiplication of the frequency (days/week), duration (minutes/day), and a corresponding metabolic equivalent value (MET; e.g., 3.3 for walking, 4.0 for moderate and 8.0 for vigorous physical activity in the work domain). Second, physical activity scores for work, domestic/yard work, and LTPA were computed by summing scores across walking, moderate (not including walking score), and vigorous intensity physical activity. The transportation physical activity score was computed by summing scores from walking and cycling. Finally, a total physical activity score (MET $\times$ minutes/week) was computed as a sum of total work, transportation, domestic/yard work, and LTPA.

The physical activity latent factor in our structural analyses was defined by three indicators (i.e., walking, moderate and vigorous physical activity). The scores were 
square root transformed before analysis since the data were positively skewed with large standard deviations in relation to the means. The factor loadings for these three indicators were $.30, .46$, and .62 , respectively. Although the factor loading for the walking was only at .30, we believe it was important to include walking as an indicator of total physical activity scores.

Predictors. Supportive workplace environments were measured with the Chinese version of the 6-item Perceived Workplace Environment Scale (PWES-C). The PWES-C assessed workers' perceptions of each of the six workplace environment dimensions of an ecological workplace physical activity model, including individual, social, organizational, community, policy, and physical environment (Prodaniuk et al., 2004). Six items refer to six dimensions of perceived workplace environment relative to PA. A sample item from the individual dimension is, "How much information is provided in your workplace educating and/or encouraging employees about physical activity?" Ratings for the scale were provided on a 5-point scale ranging from 1 (none) to 5 (a great amount). A higher score reflects a more supportive workplace environment for physical activity. The English version of the PWES has demonstrated satisfactory reliability and content and construct validity in a sample of 897 employees (Prodaniuk et al.). For this study, Cronbach's $\alpha$ of the PWES-C was .88. For the structural analyses described below, the supportive workplace environments latent factor was defined by four indicators from the six items. Based on the results of the CFA and conceptual considerations, the first indicator was formed by averaging the items 1 to 3 , but the second, third, and fourth were represented by the items 4,5 , and 6 , respectively. The factor loadings for these four indicators were $.79, .63, .79$, and .74 , respectively. 
Job strain was measured by the Psychological Job Demands and Job Control Scale, derived from the Chinese Version of the Job Content Questionnaire (C-JCQ) (Cheng, Luh, \& Guo, 2003; Yeh, Cheng, Chen, \& Chiu, 2008). The 7-item Psychological Job Demands Scale was used to assess workload, pace of work, and organizational constraints (sample item: "My work requires me to do things very quickly"). A 9-item Job Control Scale was used to measure skill discretion (six items; sample item: "My work requires me to learn new things") and decision authority (three items; sample item: "My work allows me to make my own decisions") (Yeh et al.). Each item was rated on a 4point scale $(1=$ strongly disagree to $4=$ strongly agree $)$. Negative items were reverse scored so that higher scores indicated more control over work or more demanding work. A sum of weighted item scores and a standardized score were calculated for both subscales, respectively (Yeh et al.). Evidence of adequate reliability and construct validity of both subscales has been reported in two samples of Taiwanese workers (Cheng et al.; Yeh et al.). For this study, Cronbach's $\alpha$ was .84 for Psychological Job Demands Scale and .78 for Job Control Scale. For the structural analyses described below, to reflect the job strain, one observed variable was formed by the ratio of job demands to job control, with higher scores indicating higher degrees of job-related mental strain.

Self-efficacy for physical activity was measured with the 15-item Scale of Exercise Self-Efficacy (in Chinese) (Kao et al., 2002), which assessed the respondent's confidence in engaging in regular physical activity (i.e., moderate or vigorous physical activity for 30 minutes a day, at least 10 minutes at a time, 3 days a week) over the next three months in different situations or the presence of barriers (e.g., "When my family is in need of my help"; "When I have a heavy workload"). Participants rated their 
confidence on a 6 -point scale $(1=$ not at all confident to $6=$ completely confident $)$. Higher scores indicated more confidence in overcoming barriers to engage in regular physical activity. The scale used in this study replaced the term "exercise" with "physical activity" to reflect the outcome measure. Moreover, the physical activity definition and examples of various activities in the context of the physical activity definition were added to this scale. Evidence of satisfactory reliability and content and construct validity of the scale was demonstrated in sedentary workers at a telecommunications company in Taiwan (Kao et al.). Cronbach's $\alpha$ for this study was .93 . The self-efficacy for physical activity latent factor was defined by three indicators, each consisting of the mean of five items from the scale. The factor loadings for these three indicators were $.88, .96$, and .92 , respectively.

Outcome expectations for physical activity were measured with the Chinese version of the 25-item Positive and Negative Outcome Expectations Scale (PNOES-C). The PNOES-C assessed the expected outcomes of engaging in regular physical activity (as defined above) over the next three months. The PNOES-C is comprised of two subscales: positive (12 items; sample item: "I will build up my muscle strength") and negative (13 items; sample item: "It will be too time consuming") outcomes from engaging in regular physical activity, arranged in a 5 -point scale $(1=$ not at all likely to 5 $=$ extremely likely). Following each expected outcome of engaging in regular physical activity, respondents were then asked to indicate the value (personal importance) of each outcome on a 5 -point scale $(1=$ not at all important to $5=$ extremely important $)$. A rating of outcome likelihood was multiplied by its corresponding rating of outcome value (Rovniak et al., 2002). Negative items were reverse scored. A mean score was then 
computed and included both positive and negative items. Higher scores indicated higher positive outcome expectations of engaging in regular physical activity. For the structural analyses described below, to reflect the outcome expectations for physical activity, one observed variable was formed by the mean score described above. Since the study population was IT professionals (non-students), the original item 22 ("It will take away from the time I have for my schoolwork") was modified by substituting "work" for "schoolwork." Moreover, the scale replaced the term "exercise" with "physical activity" to reflect the outcome measure. Regular physical activity and moderate and vigorous intensity physical activity were defined in the scale. Evidence of satisfactory reliability of both subscales (English version) has been reported in a sample of 277 undergraduate students (Rovniak et al.). For this study, Cronbach's $\alpha$ for the PNOES-C was .88 for the positive and .86 for the negative subscales.

Demographic and background information included age, gender, education, marital status, job title, job type, seniority, work hours, weight, height, perceived health, smoking (two items from the Health Assessment developed by the University of Michigan Heath Management Research Center), and historical physical activity (Norman et al., 2001; Orsini et al., 2007). Items originally written in English were translated into Chinese using the committee approach described earlier. For the structural analyses described below, seven covariates consisting of seniority, perceived health, work hours, historical physical activity, gender, site $\mathrm{B}$, and site $\mathrm{C}$ (with site $\mathrm{A}$ as the reference category) were treated as control variables. Seniority was assessed using the respondents' report of the number of years employed in their current position. Perceived health was measured with one item from the general health domain of the SF-36 Taiwan version 
(Lu, Tseng, \& Tsai, 2003). Participants were asked to answer the following question: "In general, would you say your health is excellent, very good, good, fair, or poor?" Work hours required the respondents to answer the question "During the last whole week you worked, how many hours did you work (including working at home or on weekends)?" Gender was coded as a dummy variable with a score of "0" assigned to respondents who were males and a score of " 1 " assigned to females. Companies were identified as sites A, $\mathrm{B}$, and $\mathrm{C}$.

A Chinese version of the Historical Physical Activity Questionnaire (HPAQ-C) was used to assess historical physical activity and inactivity at age 15, 20 and 30 . Respondents were asked to indicate one of six predefined activity levels for work/occupation (from mostly sitting down to heavy manual labor); one of five to six predefined activity time categories for walking/bicycling (from hardly ever to $>1.5$ $\mathrm{hr} /$ day), home/household work (from $<1 \mathrm{hr} /$ day to $>8 \mathrm{hr} /$ day), using computer/watching TV/reading (leisure time inactivity, from $<1 \mathrm{hr} /$ day to $>6 \mathrm{hr} /$ day), and exercise (from $<$ $1 \mathrm{hr} /$ week to $>5 \mathrm{hr} /$ week); and the number of hours spent sleeping per day at age 15, 20 and 30 (Norman et al., 2001; Orsini et al., 2007). As described by Norman et al., specific activity scores were calculated by multiplying the corresponding intensity (MET, $\mathrm{kcal} / \mathrm{kg} \times \mathrm{h}$ ) by reported duration (hours). Self-reported time was corrected to 24 hours, by adding hours (if the sum of self-reported time was $<24 \mathrm{~h}$ ) or deleting hours (if overreported $>24 \mathrm{~h}$ ). Based on the assumption that underestimation of time might be due to some common activities not asked in the questionnaire, the "correction time" was multiplied by an intensity factor of 2.0 METs that corresponded to the mean of self-care or walking at home (2.5 METs) and sitting, eating, or transportation (1.5 METs) (Norman 
et al.). Three total daily HPA scores (MET $\times$ h/day) (at age 15, 20 and 30, respectively) were then computed by adding all specific types of activity together. However, because not all of the respondents in this study were over the age of 30 , we could not use all three HPA scores described above as three indicators of the latent HPA construct. Instead, scores of HPA at age 20 were used for subjects aged 24-34, whereas scores of HPA at age 30 were used for subjects aged 35-60. In this way, we assessed respondents' past physical activity over at least five years which can be considered historical. HPA at age 15 was not an appropriate timeframe for assessing historical physical activity because in Taiwan, adolescents aged 15 are in the third (last) grade of junior high school and are expected to study hard to enter a good senior high school by taking the entrance exam. Thus, adolescents at age 15 in Taiwan are not encouraged to engage in physical activity. 
Table 4.1 Descriptive Statistics and Internal Consistency Reliabilities for the Scales

\begin{tabular}{lcccrc}
\hline \multicolumn{1}{c}{ Scale } & $\boldsymbol{N}$ & \multicolumn{1}{c}{$\boldsymbol{\alpha}$} & \multicolumn{1}{c}{$\boldsymbol{M}$} & \multicolumn{1}{c}{$\boldsymbol{S D}$} & \multicolumn{1}{c}{ Range } \\
\hline Perceived Workplace Environment Scale-C & 575 & .88 & 2.70 & .81 & $1.0-5.0$ \\
Psychological Job Demands Scale & 576 & .84 & 2.80 & .45 & $1.0-4.0$ \\
Job Control Scale & 576 & .78 & 2.77 & .39 & $1.3-3.9$ \\
Positive Outcome Expectations Scale-C & 570 & .88 & 11.17 & 4.39 & $2.1-25.0$ \\
Negative Outcome Expectations Scale-C & 570 & .84 & 5.97 & 2.92 & $1.0-22.5$ \\
Scale of Exercise Self-Efficacy & 569 & .93 & 2.67 & .97 & $1.0-6.0$ \\
International Physical Activity & 576 & - & 1949 & 2067 & $0-12600$ \\
$\quad$ Questionnaire-Taiwan & & & & & \\
HPAQ-C at age 15 & 510 & - & 40.02 & 4.75 & $25.3-57.4$ \\
HPAQ-C at age 20 & 510 & - & 39.90 & 5.05 & $27.5-57.9$ \\
HPAQ-C at age 30 & 404 & - & 35.85 & 4.43 & $25.3-58.1$ \\
\hline
\end{tabular}

Note. Dashes indicate the Cronbach's $\alpha$ was not estimated. $\mathrm{C}=\mathrm{Chinese}$, HPAQ $=$ Historical Physical Activity Questionnaire.

Table 4.2 Factor Loadings and Error for the Measured Variables (Indicators) of Latent Factors Used in the Confirmatory Factor Analysis Measurement Model

\begin{tabular}{lccc}
\hline Latent factor and its corresponding indicators & Factor loading & SE & Error \\
\hline Supportive Workplace Environment & & & \\
$\mathrm{SW}_{1}$ & .79 & .05 & .61 \\
$\mathrm{SW}_{2}$ & .63 & .06 & .78 \\
$\mathrm{SW}_{3}$ & .79 & .06 & .61 \\
$\mathrm{SW}_{4}$ & .74 & - & .67 \\
Self-Efficacy for Physical Activity & & & \\
$\mathrm{SE}_{1}$ & .88 & .03 & .48 \\
$\mathrm{SE}_{2}$ & .96 & .02 & .27 \\
$\mathrm{SE}_{3}$ & .92 & - & .39 \\
Physical Activity & & & \\
Walking & .30 & .11 & .95 \\
Moderate physical activity & .46 & .14 & .89 \\
Vigorous physical activity & .62 & - & .78 \\
\hline
\end{tabular}

Note. Dashes indicate the standard error was not estimated. $\mathrm{SW}_{1}$ to $\mathrm{SW}_{4}$ and $\mathrm{SE}_{1}$ to $\mathrm{SE}_{3}$ are indicators of the respective latent factor. All the free factor loadings and error variances are statistically significant at $p<.001$. Fit indices for measurement model: Satorra-Bentler Scaled $\chi^{2}(\mathrm{df}=32, \mathrm{~N}=576)=37.1, p=.24$; nonnormed fit index $=.99$; comparative fit index $=.99$; Bollen's (IFI) fit index $=.99$; root mean-square error of approximation $=.02(90 \% \mathrm{CI}=.00-.04)$. 


\section{Overview of Analytic Procedures}

Prior to data analyses, data were examined for accuracy, missing data, outliers, normality, linearity, and homoscedasticity. Multivariate normality was evaluated using Mardia's normalized estimate which assesses the degree of kurtosis in the data. Bentler (2006) suggests that values greater than 3 are indicative of nontrivial positive kurtosis; a multivariate normal data has a normalized estimate of 3. Tests for multivariate kurtosis showed that our Mardia's normalized estimate was 14.89, indicating that the data has violated the assumption of multivariate normality. As a result, we employed the SatorraBentler (S-B) scaling method to handle non-normal data (Finney \& DiStefano, 2006).

SPSS 18.0 for Windows was used for descriptive data analyses. Frequencies and descriptive statistics were performed to describe sample characteristics. T-tests and chisquare tests were conducted to determine if there was a statistically significant difference between qualified and disqualified groups, and the difference between respondents with complete data and those with incomplete data. One way analysis of variance (ANOVA) or chi-square tests were used to examine any differences across company groups. Bivariate correlations were used to examine relationships between variables. Internal consistency reliability for each survey instrument was assessed using Cronbach's $\alpha$.

Overall, incomplete data rates for most variables were less than $1 \%$, with the exception of incomplete data from the IPAQ-Taiwan $(n=41)$ and HPAQ-C $(n=59)$ instruments, respectively. To address this situation we followed guidelines suggested by IPAQ Research Committee (2005). Accordingly, respondents whose IPAQ data were missing for time or days were removed from the dataset $(n=41)$. When respondents with incomplete data $(n=74)$ were compared to those with complete data $(n=502)$, results 
revealed that those with incomplete data displayed a trend toward higher levels of job strain and working longer hours $(p<.05)$. To account for this difference, we imputed missing values using the modern technique — expectation maximization (EM) algorithm of the EQS software (Bentler, 2006) so that all cases $(N=576)$ were analyzed in the structural analyses. The EM algorithm generates a sequence of parameter estimates via a two-step iterative process: expectation (E) and maximization (M). "The E step effectively imputes the missing values in each case by the predictions from the regression of the missing variables on the observed variables, with coefficients based on current estimates of the parameters. The M step estimates the mean and covariance matrix from the filledin data, with corrections for the covariance matrix for imputing predicted means" (Little \& Schenker, 1995, p. 52).

Possible company effects were taken into account because ANOVA showed differences among companies in terms of supportive workplace environments, $\mathrm{F}(2,573)$ $=76.4, p<.001$. Specifically, respondents from sites $\mathrm{B}$ and $\mathrm{C}$ perceived more support of physical activity provided at their site than those from site A. Two dummy variables (i.e., site $\mathrm{B}$ and site $\mathrm{C}$ ) were created and included in the model as covariates, with site $\mathrm{A}$ as the reference group. Site B was coded with a score of 1 if site $=B$, and a score of $0=$ otherwise. Site $\mathrm{C}$ was coded with a score of 1 if site $=\mathrm{C}$, and a score of $0=$ otherwise.

The role of work environment (i.e., supportive workplace environments and job strain) and psychosocial factors (i.e., outcome expectations and self-efficacy for physical activity) on physical activity of IT professionals were described as a structural model (Figure 4.2). All of the models were tested by a structural equation modeling (SEM) analysis using the EQS 6.1 program (Bentler, 2006). All analyses were performed on 
covariance matrices using maximum likelihood estimation with the S-B scaling method (robust estimation), which adjusts the $\chi^{2}$, fit indices, and standard errors for nonnormality (Finney \& DiStefano, 2006). The SEM analysis provides simultaneous estimation of the hypothesized regressions using the estimated covariance matrix computed from a set of observed (measured) variables. The estimated covariance matrix is also used to assess the goodness of fit between the data and the model (Vinokur, 2005). We used the S-B $\chi^{2}$ and S-B scaled indices: nonnormed fit index (NNFI), comparative fit index (CFI), Bollen's (IFI) fit index, and root mean-square error of approximation (RMSEA) to evaluate the fit of the models (Hu \& Bentler, 1999; Yu \& Muthén, 2002). For fit indices, a value $\geq .95$ and for the misfit RMSEA index, a value $\leq .06$ indicate adequate fit. However, $\chi^{2}$ is sensitive to sample size, so it is difficult to obtain a nonsignificant $\chi^{2}$ when the sample size is large (Hu \& Bentler; Yu \& Muthén). To compare models with robust estimation, we used calculations based on work by Satorra and Bentler (2001) to correct the $\chi^{2}$ difference test for the S-B scaled $\chi^{2}$. A modification index, known as Lagrange Multiplier (LM) test for adding parameters was considered. The best-fitting measurement model served as a foundation for testing the structural model.

In the gender comparison model (Figure 4.3), for each multi-item scale that was treated as a single indicator for its corresponding latent factor, we corrected for random measurement error (i.e., unreliability of the scale) by setting the random error variance associated with each factor to its variance multiplied by the quantity one minus its estimated reliability; namely, error $=$ variance $\times(1-\alpha)($ Bollen, 1989). 
Before estimating the model, multiple regression analyses were performed to select important covariates. Only variables that showed statistical significance with the outcome variable were included in the model, except two dummy variables—site B and site $\mathrm{C}$ due to reasons described previously. Pearson correlations among the covariates were employed to find statistically significant correlations to be included in the model. Mediation effects were tested with SEM using steps outlined by Baron and Kenny (1986). The significance level was set at $p<.05$. Our sample size for each SEM analysis met requirements for minimal sample of $5-10$ cases per model parameter recommended by Bentler and Chou (1987).

\section{Results}

\section{Descriptive Information}

The usable sample consisted of 576 Taiwanese IT professionals from three IT companies. The respondents' age ranged from 24 to $60(M=33.7, S D=6.1)$. The sample included 467 (81\%) men and 109 (19\%) women. Nearly 61\% of the respondents reported that they engaged in recommended levels of physical activity (e.g., moderate physical activity for 30 minutes a day, at least 10 minutes at a time, 5 days a week or vigorous physical activity for 20 minutes a day, 3 days a week). However, $8.2 \%$ did not engage in any physical activity for at least 10 minutes at a time. Average sitting time on a weekday and weekend day was $7.8(S D=2.9 ;$ range $=.5-16)$ and $6.4(S D=3.4$; range $=.5-16)$ hours per day, respectively. Sample characteristics by gender and site are described in Table 4.3 and Table 4.4, respectively. The high proportion of male workers in our sample mirrored the gender distribution of the participating IT companies and also reflected the fact that the IT service business is one of the most male-intensive industries. Table 4.5 
presents the means, standard deviations, and zero-order correlations among all variables included in the model. 
Table 4.3 Sample Characteristics by Gender

\begin{tabular}{lrrrr}
\hline & \multicolumn{2}{c}{ Men $(\boldsymbol{n}=\mathbf{4 6 7})$} & \multicolumn{2}{c}{ Women $(\boldsymbol{n}=\mathbf{1 0 9})$} \\
& \multicolumn{1}{c}{ Variable } & \multicolumn{1}{c}{$\boldsymbol{S D}$} & \multicolumn{1}{c}{$\boldsymbol{M}$} & \multicolumn{1}{c}{$\boldsymbol{S D}$} \\
\hline Age (years) & 33.8 & 6.1 & 33.1 & 5.9 \\
Seniority (years) & 5.4 & 4.9 & 5.8 & 5.5 \\
Work hours (h/wk) & 49.5 & 9.9 & 47.6 & 8.7 \\
IPAQ total physical activity $($ MET $\times$ min/wk) & 2067 & 2167 & 1441 & 1476 \\
Work & 672 & 1372 & 212 & 500 \\
Transportation & 163 & 384 & 238 & 428 \\
Domestic/yard work & 382 & 684 & 406 & 775 \\
Leisure time physical activity & 849 & 1320 & 584 & 833 \\
Sitting hours (h/day) & 7.3 & 2.6 & 8.2 & 2.3 \\
HPA at age 15 (MET $\times \mathrm{h} / \mathrm{d})$ & 40.3 & 4.8 & 39.0 & 4.6 \\
HPA at age 20 (MET $\times \mathrm{h} / \mathrm{d})$ & 40.2 & 5.0 & 38.7 & 5.0 \\
HPA at age 30 $($ MET $\times \mathrm{h} / \mathrm{d})$ & 36.0 & 4.5 & 35.1 & 4.1 \\
\hline
\end{tabular}

Marital status

Married or cohabiting

Frequency (percentage)

Never married

43.2

36.1

Divorced/separated

56.3

63.0

.4

.9

Education

Senior high/vocational school

.2

.9

Junior college

13.3

13.8

College/university

49.1

59.6

Graduate school and above

37.3

25.7

Job title

Assistant engineer $\quad .9$

Associate engineer

1.5

4.6

Engineer

72.4

0

Senior/chief/senior advisory engineer

5.4

75.2

Supervisor/section manager/chief

3.9

1.8

(Associate) manager, skilled/project/senior manager

15.2

3.7

(Associate) director

.9

13.8

Body mass index $\left(\mathrm{kg} / \mathrm{m}^{2}\right)$

Underweight $(<18.5)$

Normal weight $(18.5-23.99)$

1.3

.9

Overweight (24.0 - 26.99)

46.5

15.6

Obese $(\geq 27.0)$

34.2

70.6

10.1

Perceived health

Poo

8.4

3.7

Fair

47.4

9.2

Good

31.3

50.5

Very good

11.6

26.6

Excellent

1.3

11.0

Smoking status

Current nonsmoker

81.6

2.8

Current smoker

15.6

94.5

Did not answer

$\begin{array}{rr}1.8 & 3.7\end{array}$

IPAQ physical activity categories

Low active

38.8

40.4

Moderate activity

38.3

44.0

High active

22.9

15.6

Note. Data on this table are based on non-missing cases. HPA = Historical physical activity. IPAQ = International Physical Activity Questionnaire-Taiwan. 
Table 4.4 Sample Characteristics by Site

\begin{tabular}{|c|c|c|c|c|c|c|}
\hline \multirow[b]{2}{*}{ Variable } & \multicolumn{2}{|c|}{ Site A $(n=169)$} & \multicolumn{2}{|c|}{ Site B $(n=196)$} & \multicolumn{2}{|c|}{ Site C $(n=211)$} \\
\hline & $\boldsymbol{M}$ & $S D$ & $\boldsymbol{M}$ & $S D$ & M & $S D$ \\
\hline Age (years) & 36.4 & 7.9 & 32.1 & 4.3 & 33.1 & 5.1 \\
\hline Seniority (years) & 7.7 & 7.0 & 3.8 & 2.8 & 5.3 & 4.0 \\
\hline Work hours (h/wk) & 47.9 & 7.5 & 51.2 & 11.7 & 48.1 & 8.9 \\
\hline IPAQ total physical activity (MET $\times \mathrm{min} / \mathrm{wk}$ ) & 1799 & 1941 & 1841 & 2179 & 2168 & 2050 \\
\hline Work & 563 & 1357 & 347 & 897 & 824 & 1438 \\
\hline Transportation & 249 & 405 & 139 & 430 & 155 & 339 \\
\hline Domestic/yard work & 330 & 629 & 358 & 686 & 458 & 765 \\
\hline Leisure time physical activity & 656 & 939 & 996 & 1629 & 729 & 1014 \\
\hline Sitting hours (h/day) & 7.4 & 2.4 & 8.1 & 2.4 & 6.8 & 2.6 \\
\hline HPA at age $15(\mathrm{MET} \times \mathrm{h} / \mathrm{d})$ & 39.6 & 4.8 & 40.4 & 4.9 & 40.0 & 4.6 \\
\hline HPA at age $20($ MET $\times \mathrm{h} / \mathrm{d})$ & 39.6 & 4.9 & 39.5 & 5.1 & 40.4 & 5.1 \\
\hline HPA at age $30(\mathrm{MET} \times \mathrm{h} / \mathrm{d})$ & 36.1 & 4.5 & 34.8 & 3.4 & 36.5 & 4.9 \\
\hline
\end{tabular}

Frequency (percentage)

\begin{tabular}{|c|c|c|c|}
\hline \multicolumn{4}{|l|}{ Marital status } \\
\hline Married or cohabiting & 46.7 & 37.9 & 41.7 \\
\hline Never married & 53.3 & 61.5 & 57.3 \\
\hline Divorced/separated & 0 & .5 & .9 \\
\hline \multicolumn{4}{|l|}{ Education } \\
\hline Senior high/vocational school & .6 & 0 & .5 \\
\hline Junior college & 23.8 & 11.7 & 6.6 \\
\hline College/university & 51.8 & 61.2 & 41.2 \\
\hline Graduate school and above & 23.8 & 27.0 & 51.7 \\
\hline \multicolumn{4}{|l|}{ Job title } \\
\hline Assistant engineer & 2.4 & 2.0 & .5 \\
\hline Associate engineer & 0 & 0 & 3.3 \\
\hline Engineer & 68.0 & 68.4 & 81.0 \\
\hline Senior/chief/senior advisory engineer & 1.8 & 9.7 & 2.4 \\
\hline Supervisor/section manager/chief & 1.8 & 6.6 & 2.8 \\
\hline (Associate) manager, skilled/project/senior manager & 23.1 & 13.3 & 10.0 \\
\hline (Associate) director & 3.0 & 0 & 0 \\
\hline \multicolumn{4}{|l|}{ Body mass index $\left(\mathrm{kg} / \mathrm{m}^{2}\right)$} \\
\hline Underweight $(<18.5)$ & 3.0 & 5.6 & 3.3 \\
\hline Normal weight $(18.5-23.99)$ & 50.0 & 56.6 & 46.7 \\
\hline Overweight $(24.0-26.99)$ & 31.0 & 21.4 & 36.2 \\
\hline Obese $(\geq 27.0)$ & 16.1 & 16.3 & 13.8 \\
\hline \multicolumn{4}{|l|}{ Perceived health } \\
\hline Poor & 6.5 & 13.8 & 5.2 \\
\hline Fair & 47.3 & 53.3 & 43.6 \\
\hline Good & 30.8 & 24.6 & 35.5 \\
\hline Very good & 14.2 & 5.6 & 14.7 \\
\hline Excellent & 1.2 & 2.6 & .9 \\
\hline \multicolumn{4}{|l|}{ Smoking status } \\
\hline Current nonsmoker & 85.8 & 83.2 & 83.4 \\
\hline Current smoker & 8.9 & 15.3 & 14.2 \\
\hline Did not answer & 5.3 & 1.5 & 2.4 \\
\hline \multicolumn{4}{|l|}{ IPAQ physical activity categories } \\
\hline Low active & 36.7 & 43.4 & 37.0 \\
\hline Moderate activity & 45.0 & 38.8 & 35.5 \\
\hline High active & 18.3 & 17.9 & 27.5 \\
\hline
\end{tabular}


Table 4.5 Means, Standard Deviations, and Zero-Order Correlations Among Variables Included in the Model

\begin{tabular}{|c|c|c|c|c|c|c|c|c|c|c|c|c|c|c|}
\hline & 1 & 2 & 3 & 4 & 5 & 6 & 7 & 8 & 9 & 10 & 11 & 12 & 13 & 14 \\
\hline $\begin{array}{l}\text { 1. Supportive workplace } \\
\text { environments }\end{array}$ & & & & & & & & & & & & & & \\
\hline 2. Job strain & $-.21 * * *$ & & & & & & & & & & & & & \\
\hline $\begin{array}{l}\text { 3. Outcome expectations } \\
\text { for PA }\end{array}$ & .03 & $-.10^{*}$ & & & & & & & & & & & & \\
\hline 4. Self-efficacy for PA & $.12 * *$ & $-.16^{* * *}$ & $.26^{* * *}$ & & & & & & & & & & & \\
\hline 5. Walking ${ }^{\mathrm{a}}$ & -.01 & -.01 & .07 & $.12 * *$ & & & & & & & & & & \\
\hline 6. Moderate $\mathrm{PA}^{\mathrm{a}}$ & .07 & -.05 & $.17 * * *$ & $.17^{* * *}$ & $.18 * * *$ & & & & & & & & & \\
\hline 7. Vigorous $\mathrm{PA}^{\mathrm{a}}$ & $.14 * * *$ & -.04 & $.15^{* * *}$ & $.24 * * *$ & $.18^{* * *}$ & $.28 * * *$ & & & & & & & & \\
\hline 8. Seniority & $-.12 * *$ & -.06 & .05 & $.12 * *$ & $.13 * *$ & $.09 *$ & .02 & & & & & & & \\
\hline 9. Perceived health & .07 & $-.13 * *$ & .06 & $.25^{* * *}$ & .07 & $.11^{*}$ & $.16^{* * *}$ & $.08^{*}$ & & & & & & \\
\hline 10. Work hours & -.05 & $.15^{* * *}$ & -.06 & -.03 & -.06 & -.02 & $-.10^{*}$ & .03 & $-.14 * * *$ & & & & & \\
\hline 11. Historical PA & .05 & .04 & $.11^{* *}$ & .08 & $.16^{* * *}$ & $.15^{* * *}$ & $.12 * *$ & .03 & .08 & .08 & & & & \\
\hline 12. Gender ${ }^{\mathrm{b}}$ & -.05 & .04 & -.08 & -.06 & .02 & -.07 & $-.18^{* * *}$ & .03 & -.01 & -.08 & -.05 & & & \\
\hline 13. Site $B^{c}$ & $.15^{* * *}$ & .06 & .04 & .01 & $-.17 * * *$ & -.01 & .03 & $-.24 * * *$ & $-.17 * * *$ & $.16^{* * *}$ & -.05 & -.04 & & \\
\hline 14. Site $C^{d}$ & $.28 * * *$ & -.07 & -.07 & .01 & $.13^{* *}$ & .03 & .06 & -.04 & $.12 * *$ & -.08 & $.12^{* *}$ & $-.12 * *$ & $-.55^{* * *}$ & \\
\hline$M$ & 2.70 & 1.10 & 12.61 & 2.66 & 17.40 & 20.26 & 16.21 & 5.49 & 2.50 & 49.12 & 38.35 & .19 & .34 & .37 \\
\hline$S D$ & .81 & .44 & 2.70 & .97 & 16.39 & 17.71 & 19.82 & 5.01 & .86 & 9.69 & 4.83 & .39 & .47 & .48 \\
\hline
\end{tabular}

Note. PA = Physical Activity.

${ }^{\mathrm{a}}$ scores were square root transformed before analysis since the data were positively skewed. ${ }^{\mathrm{b}}$ Gender categories include $0=$ male, $1=$ female. ${ }^{\mathrm{c}}$ Site B categories include 1 if Site $=\mathrm{B}, 0=$ otherwise (i.e., if Site $=\mathrm{A}$ or C). ${ }^{\mathrm{d}}$ Site $\mathrm{C}$ categories include 1 if Site $=\mathrm{C}, 0=$ otherwise (i.e., if Site $=\mathrm{A}$ or B). Site A was chosen as the reference group in the structural analyses.

$* p<.05 . * * p<.01 . * * * p<.001$. 


\section{Measurement Model}

Prior to testing the structural model specified in Figure 4.2, the adequacy of the measurement model of the three latent factors (i.e., supportive workplace environments, self-efficacy for physical activity, and physical activity) was tested. Table 4.2 presents the pattern of factor loading for each measured variable (indicator), ranged from .30 to .96 . The test of the measurement model showed a good fit to the actual data, S-B $\chi^{2}$ (df $=32, N=576)=37.1, p=.24 ; \mathrm{NNFI}=.99, \mathrm{CFI}=.99, \mathrm{IFI}=.99$, and $\mathrm{RMSEA}=.02(90 \%$ CI: .00-.04). It was thus used in testing the structural model described below.

\section{Testing the Proposed Model of Physical Activity among IT Professionals}

The structural model was tested to address the four key hypotheses described earlier. Table 4.6 summarizes the fit statistics across the six models tested using the robust estimation method. According to the LM test, three directional paths (one from self-efficacy to outcome expectations, one from job strain to self-efficacy, and another from site $\mathrm{C}$ to outcome expectations) and one correlation (between work hours and historical physical activity) that are conceptually and/or empirically meaningful were added to the proposed model individually. We found the initial simple model fit reasonably well, although the nested model comparisons that we performed using calculations based on work by Satorra and Bentler (2001) indicated that all of the modified models fit significantly better than the initial model at $p<.05$ or below. Figure 4.2 displays the results of the structural modeling analysis to test the final model and estimates of its parameters. The values along the paths represent standardized path coefficients $(\beta)$. The final model showed a good fit to the data: S-B $\chi^{2}(\mathrm{df}=126, N=576)$ 


$$
=272.3, p=.00 ; \mathrm{NNFI}=.94, \mathrm{CFI}=.96, \mathrm{IFI}=.96 \text {, and } \mathrm{RMSEA}=.05(90 \% \mathrm{CI}: .04-.05) .
$$

The final model accounted for $31 \%$ of the variance in physical activity.

Table 4.6 Fit Statistics of the Structural Equation Model of the Effects of Work Environment and Psychosocial Factors on Physical Activity for the Entire Sample

\begin{tabular}{llccccccc}
\hline Model & Path/Correlation added & S-B $\chi^{2}$ & df & $\boldsymbol{p}$ & NNFI & CFI & IFI & RMSEA \\
\hline Initial & & 316.9 & 128 & .00 & .92 & .94 & .94 & .05 \\
Second & F2 $\rightarrow$ V6 & 283.0 & 127 & .00 & .94 & .95 & .95 & .05 \\
Third & V5 $\rightarrow$ F2 & 277.7 & 126 & .00 & .94 & .95 & .95 & .05 \\
Fourth & V19 $\rightarrow$ V6 & 271.9 & 125 & .00 & .94 & .96 & .96 & .05 \\
Fifth & V15 $\rightarrow$ V16 & 266.0 & 124 & .00 & .94 & .96 & .96 & .05 \\
& $\quad$ & & & & & & & \\
Final & Path removed & & & & & & \\
& V18 $\rightarrow$ F2 & 272.3 & 126 & .00 & .94 & .96 & .96 & .05 \\
\hline
\end{tabular}

Note. $\mathrm{V}=$ variable; $\mathrm{F}=$ factor. $\mathrm{S}-\mathrm{B} \chi^{2}=$ Satorra-Bentler Scaled $\chi^{2} ; \mathrm{NNFI}=$ nonnormed fit index; $\mathrm{CFI}=$ comparative fit index; IFI = Bollen's (IFI) fit index; RMSEA = root mean-square error of approximation.

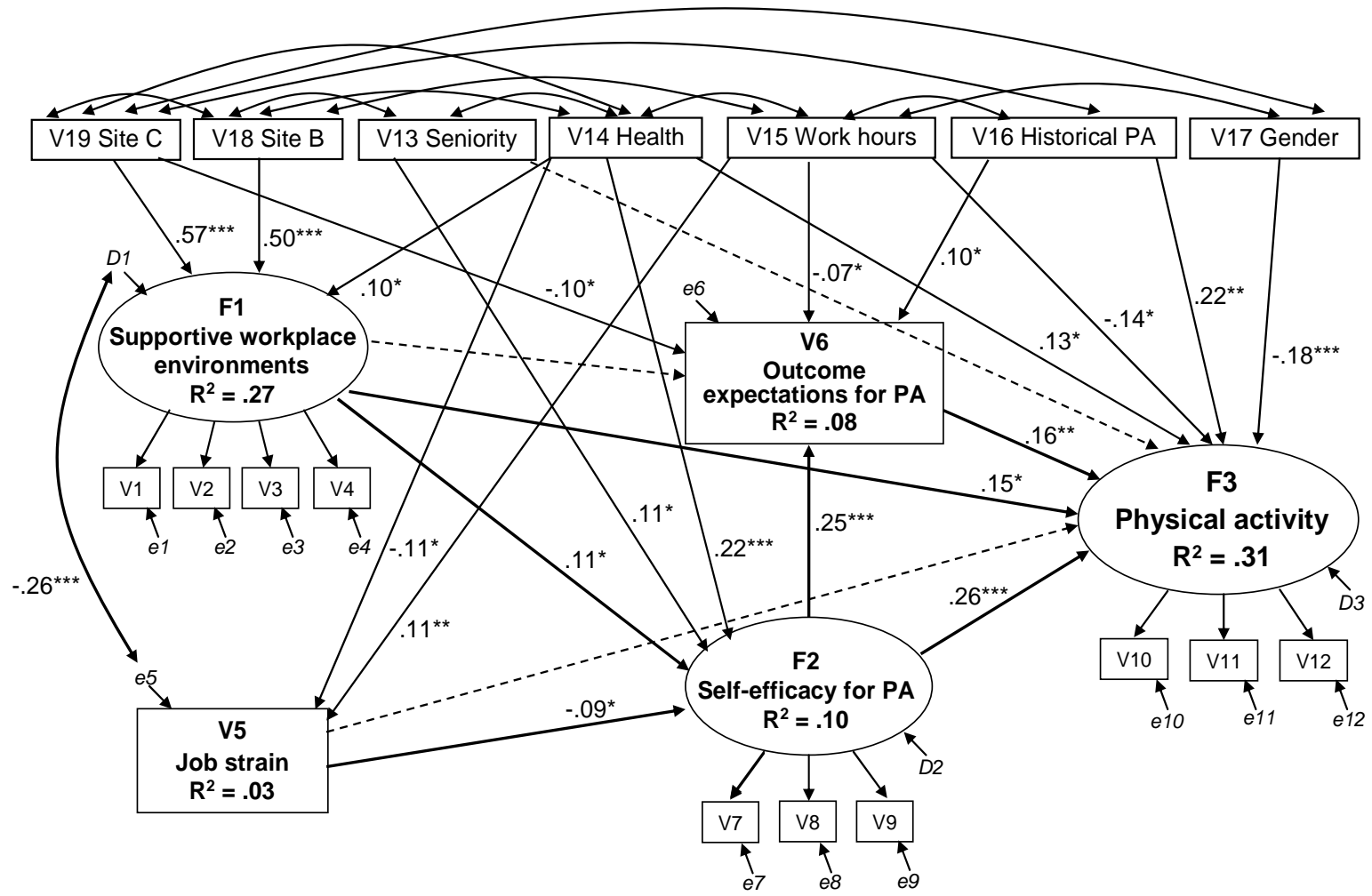

Figure 4.2. Structural equation model of the effects of work environment and psychosocial factors on physical activity: Satorra-Bentler Scaled $\chi^{2}(\mathrm{df}=126, \mathrm{~N}=576)=272.3, p=.00 ; \mathrm{NNFI}=.94 ; \mathrm{CFI}=.96 ; \mathrm{IFI}=.96$; RMSEA $=.05$. Large ellipses represent latent factors. Large and small rectangles represent observed variables. Solid and broken lines represent, respectively, statistically significant $\left({ }^{\star} p<.05,{ }^{\star \star} p<.01,{ }^{\star \star \star} p<.001\right)$ and nonsignificant paths that were included in the model. $\mathrm{PA}=$ physical activity; $\mathrm{e}=$ error; $\mathrm{D}=$ disturbance. 
The findings provide full support for Hypothesis 3, but only partial support for Hypotheses 1 and 4 in a model that included seven covariates (i.e., seniority, perceived health, work hours, historical physical activity, gender, site B, and site C). Namely, outcome expectations and self-efficacy had a significant positive effect on physical activity (H3). Supportive workplace environments had a significant positive effect on physical activity and self-efficacy (H1). Self-efficacy significantly mediated the effects of supportive workplace environments on physical activity (H4). Results failed to support the hypotheses that a supportive workplace environment has a positive effect on outcome expectations (H1); job strain has a negative effect on physical activity (H2); and outcome expectations mediate the effects of supportive workplace environments on physical activity (H4).

The effects of covariates on physical activity and/or the other constructs are also displayed in Figure 4.2. As expected, the significant predictors of higher levels of physical activity were self-efficacy $(\beta=.26, p<.001)$, historical physical activity $(\beta$ $=.22, p<.01)$, being male $(\beta=-.18, p<.001)$, outcome expectations $(\beta=.16, p<.01)$, supportive workplace environments $(\beta=.15, p<.05)$, work hours $(\beta=-.14, p<.05)$, and perceived health $(\beta=.13, p<.05)$. The significant predictors of higher self-efficacy for physical activity were perceived health $(\beta=.22, p<.001)$, supportive workplace environments $(\beta=.11, p<.05)$, seniority $(\beta=.11, p<.05)$, and job strain $(\beta=-.09, p$ $<.05)$. The significant predictors of higher outcome expectations for physical activity were self-efficacy $(\beta=.25, p<.001)$, historical physical activity $(\beta=.10, p<.05)$, site $\mathrm{C}$ $(\beta=-.10, p<.05)$, and work hours $(\beta=-.07, p<.05)$. Work hours $(\beta=.11, p<.05)$ and perceived health $(\beta=-.11, p<.05)$ were significant predictors of higher job strain. Site $\mathrm{C}$ 
$(\beta=.57, p<.001)$ and site $\mathrm{B}(\beta=.50, p<.001)$, and perceived health $(\beta=.10, p<.05)$ were significant predictors of higher supportive workplace environments. In brief, the major contributors to physical activity were self-efficacy for physical activity (total effect $=.30)$, historical physical activity (total $\mathrm{effect}=.24)$, perceived health $($ total effect $=.21)$, and supportive workplace environments (total effect $=.18$ ).

Regarding possible mediating effects, seven significant indirect effects on physical activity were found. Self-efficacy partially mediated the effect of supportive workplace environments $($ Sobel test $=2.05, p<.05)$ and perceived health $($ Sobel test $=$ $3.12, p<.01)$ on physical activity, respectively. Although higher perceptions of supportive workplace environments remained significantly related to higher levels of physical activity when self-efficacy was controlled, the magnitude of the association was reduced by $14.8 \%$ ( $\beta$ was reduced from .18, $p<.01$ to $.15, p<.05$ ). Similarly, although better perceptions of health remained significantly related to higher levels of physical activity when self-efficacy was controlled, the magnitude of the association was reduced by $28.4 \%$ ( $\beta$ was reduced from .18, $p<.01$ to $.13, p<.05$ ). Further, self-efficacy fully mediated the effect of seniority on physical activity, as seniority had no significant direct effect on physical activity when self-efficacy was controlled ( $\beta$ was reduced from .14, $p$ $<.05$ to $.10, n s$; Sobel test $=2.15, p<.05$ ). On the other hand, the influence of selfefficacy on physical activity was partially mediated by outcome expectations (Sobel test $=2.52, p=.01)$. Self-efficacy remained significantly related to higher levels of physical activity when outcome expectations was controlled; however, the magnitude of the association was reduced by $15.3 \%$ ( $\beta$ was reduced from .30, $p<.001$ to $.26, p<.001$ ). Additionally, job strain had a significant indirect effect on physical activity via self- 
efficacy $(\beta=-.03, p<.05)$, although it had no significant direct effect on physical activity in the absence of self-efficacy. Both site $\mathrm{B}(\beta=.09, p<.01)$ and site $\mathrm{C}(\beta=.08, p<.05)$ had a significant indirect effect on physical activity via supportive workplace environments, although they had no significant direct effect on physical activity in the absence of supportive workplace environments.

\section{Gender Effects}

Approximately $81 \%$ of our respondents were men. In this study, gender was a significant predictor of physical activity, indicating that men engaged in more physical activity than women. This leaves open the possibility that results in our model based on male and female subgroups may differ. To explore this possibility, due to the small number of female respondents $(n=109)$, it was necessary to limit the number of indicators for each latent factor in the model to one, except for the outcome variable. In addition, all covariates were trimmed from the final model using the male and female subgroups separately (Figure 4.3). The scale value was taken as the indicator for the latent factor, and the correction for random measurement error was handled as described earlier. In this analysis, factor loadings, variances of exogenous variables/factors, and path coefficients were constrained to be equal between male and female subgroups. This model with equality constraints between two separate groups produced a good fit to the data: $\mathrm{S}-\mathrm{B} \chi^{2}(\mathrm{df}=31, N s=467,109)=32.4, p=.40 ; \mathrm{NNFI}=.99, \mathrm{CFI}=1.0, \mathrm{IFI}=1.0$, and RMSEA $=.02(90 \% \mathrm{CI}: .00-.05)$.

Overall, results showed that men and women shared the same patterns in terms of the strength and direction of the paths in the findings presented in Figure 4.2. Of the eight path coefficients constrained to be equal in the two groups, only one was found to be 
significantly different between male and female subgroups. Hence, we released the path from supportive workplace environments to self-efficacy for both subgroups and reran the model with the modification. The latter model (Figure 4.3) with few S-B $\chi^{2}$ produced an excellent fit to the data and was significantly better than the former model at $p<.01$, $\mathrm{S}-\mathrm{B} \chi^{2}(\mathrm{df}=30, N s=467,109)=23.4, p=.80 ; \mathrm{NNFI}=1.0, \mathrm{CFI}=1.0, \mathrm{IFI}=1.0$, and RMSEA $=.00$ (90\% CI: .00 - .03). The model accounted for $19 \%$ of the variance in physical activity for men and 30\% for women. In particular, the impact of supportive workplace environments on self-efficacy was different for men $(\beta=.16, p<.001)$ and women $(\beta=-.17, n s)$, which indicated a different direction. For men, we found that supportive workplace environments also had a significant indirect effect on physical activity $(\beta=.06, p<.01)$, but not for women. Self-efficacy partially mediated the effect of supportive workplace environments on physical activity (Sobel test $=2.66, p<.001$ ). Based on these results, we concluded that the effect of gender on our findings was not substantial and that our final model presented in Figure 4.2 adequately addressed the relationships examined for both men and women respondents. 


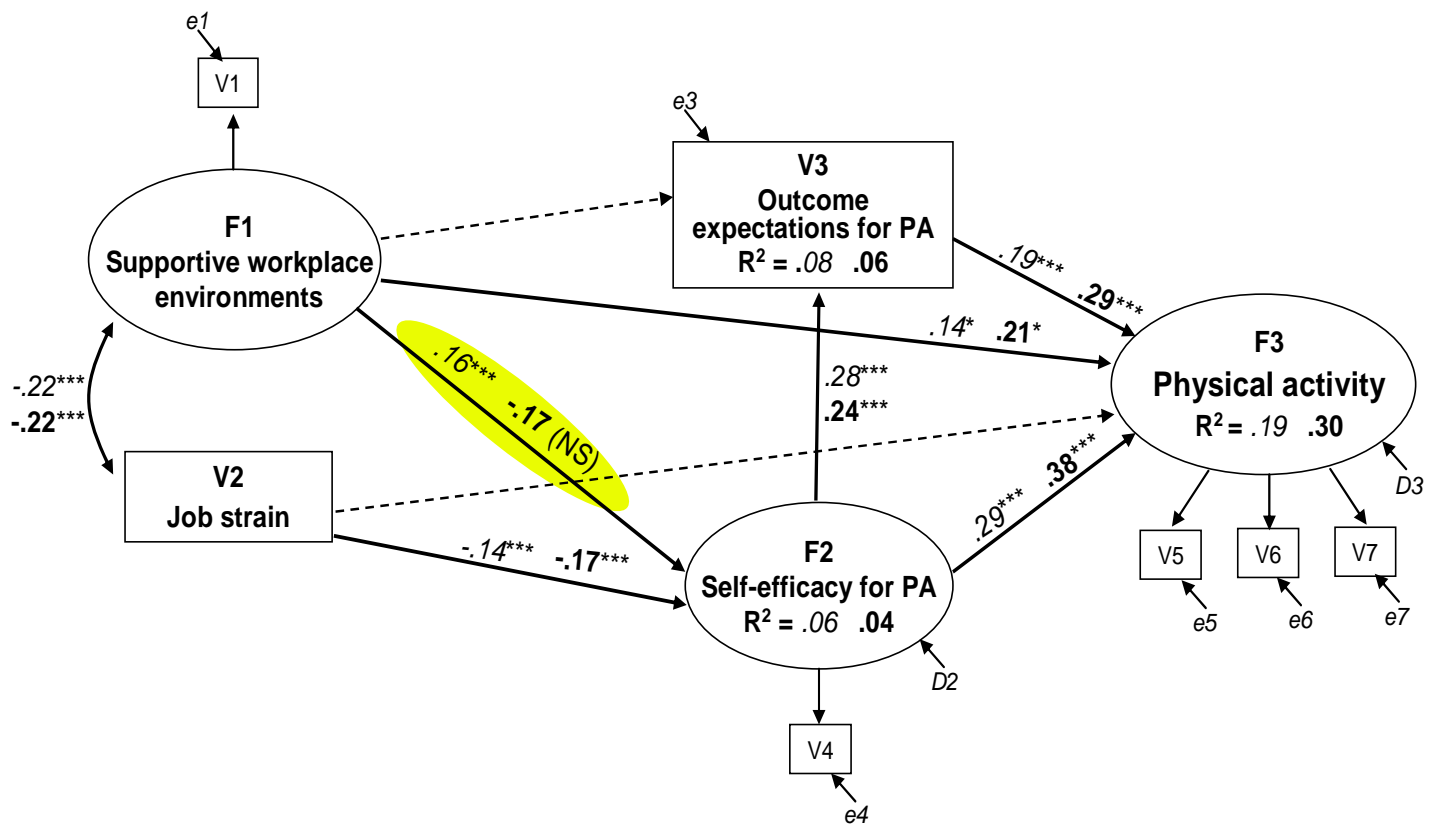

Figure 4.3. Gender comparison structural equation model of the effects of work environment and psychosocial factors on physical activity: Satorra-Bentler scaled $\chi^{2}(\mathrm{df}=30, \mathrm{Ns}=467,109)=23.4, p=.80$; NNFI = 1.0; CFI = 1.0; IFI = 1.0; RMSEA = .00. Large ellipses represent latent factors. Large and small rectangles represent observed variables. Figures in italics and bold are, respectively, for men and women. PA = physical activity; $\mathrm{e}=$ error; $\mathrm{D}=$ disturbance. ${ }^{\star} p<.05 .{ }^{* \star} p<.01 .{ }^{* \star \star} p<.001$. NS $=$ nonsignificant. 


\section{Qualitative Results}

At the end of the survey, respondents were given an opportunity to make recommendations on how to promote physical activity for IT professionals in their company. Content analysis (Berg, 1998) was used to analyze responses to this openended question and to quantify the data. The 175 responses (response rate: $23.8 \%$ ) were carefully reviewed and categorized into four broad themes and several subthemes on the basis of how frequently the theme was mentioned. The four themes that emerged and their respective subthemes are listed in Table 4.7.

Table 4.7 Participant Recommendations for Promoting Physical Activity for IT Professionals in Their Company $(\mathrm{n}=175)$

\begin{tabular}{llr}
\hline \multicolumn{1}{c}{ Themes } & \multicolumn{1}{c}{ Subthemes } & Percentage \\
\hline $\begin{array}{l}\text { Education and } \\
\text { encouragement }\end{array}$ & $\begin{array}{c}\text { - Provide information about physical activity to educate } \\
\text { and encourage workers }\end{array}$ & 6.3 \\
$\begin{array}{l}\text { Socio-cultural } \\
\text { environment }\end{array}$ & $\begin{array}{l}\text { - Engage in physical activity with others } \\
\text { - Create a supportive culture for physical activity (e.g., } \\
\text { support from management/supervisors) }\end{array}$ & 33.7 \\
Organizational & - Provide monetary and/or non-monetary incentives & 4.6 \\
support/policy & $\begin{array}{l}\text { - Offer subsidies for physical activity groups and } \\
\text { memberships at a local fitness center }\end{array}$ & 12.0 \\
& $\begin{array}{l}\text { - Create a healthy work culture (e.g., reduce workload } \\
\text { and do not encourage overtime) }\end{array}$ & 12.0 \\
& $\begin{array}{l}\text { - Issue a regulation to permit/require workers to exercise } \\
\text { at work }\end{array}$ & 10.9 \\
& $\begin{array}{l}\text { - Offer various forms of group physical activity } \\
\text { programs }\end{array}$ & 10.9 \\
& $\begin{array}{l}\text { Make physical activity engagement as part of an } \\
\text { employee evaluation }\end{array}$ & 5.1 \\
& $\begin{array}{l}\text { - Hire qualified personnel to develop feasible physical } \\
\text { activity programs }\end{array}$ & 2.9 \\
Physical & $\begin{array}{l}\text { Provide facilities for physical activity (e.g., fitness } \\
\text { center, shower rooms, bike racks, swimming pool, etc.) }\end{array}$ & 18.3 \\
& $\begin{array}{l}\text { Provide equipment for physical activity (e.g., Wii and } \\
\text { others) }\end{array}$ & 9.1 \\
\hline
\end{tabular}




\section{Discussion}

Consistent with Social Cognitive Theory, results presented in our model (Figure 4.2) indicated that supportive workplace environments, outcome expectations, and selfefficacy had a positive effect on physical activity behavior. In addition, the influence of supportive workplace environments on physical activity was partially mediated by selfefficacy. The results lend support to the general framework of Social Cognitive Theory used for developing interventions to promote physical activity in Taiwanese IT professionals.

The findings of this study support a growing body of evidence that self-efficacy is the most influential determinant of physical activity among employees (Chen \& Chang, 2004; Kaewthummanukul et al., 2006; Kao et al., 2002; Lee et al., 2005; Nishida et al., 2004; Payne et al., 2002; Prodaniuk et al., 2004; Purath, 2006; Sassen et al., 2010; Tavares et al., 2009). People with high self-efficacy expect to produce desired outcomes, whereas those with low self-efficacy expect their efforts to produce poor outcomes (Bandura, 2004). Barriers identified by the subjects in this study were similar to those reported by Chang (2007) and Lee et al. (2005), who studied Taiwanese manufacturing workers and clinical nurses, respectively.

In our structural analyses, the outcome expectations observed variable was formed by a mean score that included both positive and negative items, suggesting that higher positive outcome expectations led to higher levels of physical activity. In a separate analysis, results showed that higher positive outcome expectations $(r=.14, p$ $=.001)$ and lower negative outcome expectations $(r=-.13, p=.002)$ were correlated with increased physical activity. Due to a lack of prior research in this area, we used the 
concept of outcome expectations from Williams et al. (2005) to allow us to compare our findings with past studies that found that perceived benefits or pros of physical activity were positively related to physical activity (Kaewthummanukul et al., 2006; Nishida et al., 2004; Prodaniuk et al., 2004; Purath, 2006; Steinhardt \& Dishman, 1989; Tavares et al., 2009). In contrast, perceived barriers or cons of physical activity were negatively related to physical activity (Chen \& Chang, 2004; Kaewthummanukul et al.; Kao et al., 2002; Nishida et al.; Prodaniuk et al.; Purath, 2006; Sassen et al., 2010; Steinhardt \& Dishman,; Tavares et al.). Important positive and negative outcome expectations for physical activity identified by our participants were somewhat congruent with those reported by Steinhardt and Dishman using a U.S. sample of primarily white upper middle class workers.

In line with earlier findings (Dodson et al., 2008; Lucove et al., 2007; Prodaniuk et al., 2004), workers' perceptions of a more supportive workplace environment can contribute directly to higher levels of physical activity. Encouragingly, our participants' perceptions of supportive workplace environments were consistent with the actual existence of company resources to support employee physical activity. Lucove et al. indicated that subsidies for health club use, on-site exercise facilities, paid time for nonwork-related physical activity, and a safe place to walk outside work were positively associated with LTPA and/or work-break physical activity. The results of the present study were also similar to the findings of Prodaniuk et al. and Plotnikoff et al. (2010), who found that self-efficacy partially mediated the effect of perceived workplace environment on workplace physical activity in cross-sectional and longitudinal analyses, 
respectively. Yet, possible confounding variables were not included in their analyses, except two site dummy variables.

It is not surprising that supportive workplace environments affected men more than women, as in Taiwanese society, men are expected to be the primary breadwinners for their families. As a result, jobs have been considered more important for men than for women, and men often work longer hours than women. Because men tend to spend more time than women in the workplace, they have more opportunity to use on-site physical activity facilities or attend exercise classes after work. In contrast, married women, while employed, take on multiple roles as breadwinners, homemakers, caretakers, and nurturers that may not allow them to take full advantage of the supportive workplace environments. Although we must interpret these findings cautiously given the small sample size of the female subgroup $(n=109)$ used for multigroup analysis, the results have important implications for including supportive environments conducive to active living in workplace physical activity interventions, particularly for males.

Consistent with previous research (Landsbergis et al., 1998), we did not find a significant relation between job strain (ratio of demands to control) and physical activity in our structural analyses. Neither job demands nor job control was significantly associated with physical activity in bivariate correlations or multiple regression analyses (Kaewthummanukul et al., 2006; Landsbergis et al.; Payne et al., 2002, 2005). This is likely due to lack of variance in job strain $(M=1.10, S D=.44)$ in this particular working group. Another explanation might be that individual workers cope with job stress differently in regard to physical activity behavior. For example, some workers were better able to cope with job stress by engaging in more physical activity, whereas others 
reported working long hours and could not exercise due to time issues. However, we did find that job strain had a significant indirect effect on physical activity through selfefficacy in our structural analyses. This implies that job strain may affect physical activity indirectly by reducing one's confidence in overcoming barriers to physical activity. Likewise, Payne et al. (2002, 2005), using a sample from a U.K. computer company, expressed the same idea that job demands affected exercise indirectly by reducing one's exercise self-efficacy or perception of control over exercise. Payne et al. (2002) also suggested that job strain may disturb workers' ability to actually exercise, although it had no direct effect on intention to exercise. In other words, job strain may influence workers' confidence in their ability to engage in physical activity.

Other factors that directly influenced physical activity include historical physical activity, gender, work hours, and perceived health (Figure 4.2). Our findings are consistent with others (Conroy, Cook, Manson, Buring, \& Lee, 2005; Lee, Paffenbarger, \& Hsieh, 1992) in showing that people who were physically active during young adulthood were more likely to be active later in life. However, very few studies have examined how historical physical activity during young adulthood (ages 20-30) influences current physical activity or have assessed physical activity over a period of time longer than one year that could be considered historical. For a shorter time period, Purath (2006) found that physically active women were more likely to have a past history of sustained physical activity ( $>6$ months) than inactive women.

In addition, men were more likely than women to engage in physical activity, particularly vigorous physical activity. This finding is supported by previous studies 
(Brisson et al., 2000; Chen et al., 2008; Kaleta \& Jegier, 2005; Kouvonen et al., 2005; Steinhardt \& Dishman, 1989).

Results of this study also demonstrated that employees who worked longer hours were less likely to engage in physical activity. This finding echoes prior research (Artazcoz, Cortès, Escribà-Agüir, Cascant, \& Villegas, 2009; Schneider \& Becker, 2005). However, Lallukka et al. $(2004,2008)$ did not find a significant relationship between these two variables.

Finally, perceived health appeared to have a positive impact on physical activity, directly and indirectly, mainly via the increase in self-efficacy. This extends prior research suggesting that perceived poor health was associated with insufficient physical activity (Burton \& Turrell, 2000; Duffy, Rossow, \& Hernandez, 1996), while those who reported good health were more likely to engage in adequate physical activity (Lee, 2008). Yet, it is possible that since higher levels of physical activity contribute to positive health outcomes (Burton et al., 2005; Herman et al., 2006), individuals who engage in higher levels of physical activity have better health outcomes.

The qualitative results from this study are somewhat similar to the findings in the literature. Kruger, Yore, Bauer, and Kohl (2007) reported that the most favored physical activity services cited by 2,337 U.S. workers were fitness centers and on-site exercise classes. In addition, the most supported policy was giving paid time to exercise at work, while the most commonly cited incentives for the programs were convenient time and location. Active workers were more likely than inactive workers to cite a convenient time and location as incentives to motivate their participation in the programs. Previous qualitative work (Chang, 2007; Tavares \& Plotnikoff, 2008) also supported the argument 
that a healthy corporate culture that promotes physical activity has a positive impact on workers' motivation for physical activity engagement.

\section{Study Strengths and Limitations}

A unique feature of this study is that it used a theoretical approach to incorporate job strain variables from the demand/control model into Social Cognitive Theory to predict physical activity among Taiwanese IT professionals. In addition, to our knowledge, it is the first study in the field of worker physical activity to employ SEM with observed and latent variables to examine the complex interrelationship among independent variables, to account for measurement error (thus producing more accurate estimates of the path coefficients and test of meditational effects) (Baron \& Kenny, 1986), and to conduct multigroup analyses with men and women subsamples. Further, because our overall response rate was very high $(93.5 \%)$, the possibility of selection bias is expected to be low.

However, there are some limitations of the present study. First, due to the crosssectional design, relationships among variables should be treated with some caution and considered to be tentative.

Second, our sample was limited to IT professionals from three IT companies in northern Taiwan. This limits the generalizability of the findings to other types of businesses and different geographic locations of Taiwan.

Third, our data were based on self-report and might be subject to recall and response bias (misclassification) and contaminated by common factors such as negative affectivity (Burke, Brief, \& George, 1993) and social desirability (Weisberg, 2005). However, respondent confidentiality and anonymity were maintained by not associating 
surveys with any participant name or number and not requiring a signature on the consent form. In addition, all of the measures used have been shown to be reliable and valid in previous studies as well as in the current study. Future research may include measures of negative affectivity and/or social desirability as a covariate.

Fourth, the IPAQ long form has been found to overestimate physical activity. It is because of its assessment of the multiple domains of physical activity, compared to the IPAQ short form and other measures that assess LTPA alone (Craig et al., 2003).

Fifth, we acknowledge the difficulty in assessing historical physical activity, especially problems with recall. This might contribute to the relatively large amount of missing values resulting in reduced samples for the analysis.

Finally, the total amount of variance in physical activity accounted for in our model was modest (31\%); however, these results provide evidence to move beyond focusing on the individual level factors to consider environmental influences in a workplace context. This would require further study to replicate and generalize the current findings by including more diverse IT professionals from different businesses and industries to allow for a better understanding of the interrelationship among the factors identified in our model. This also suggests the need for future research to include such variables as social support and intention in our model, in order to enhance the explained variance for physical activity. Behavioral intention is a strong predictor of physical activity in addition to self-efficacy (Payne et al., 2002; Tavares et al., 2009).

\section{Implications for Practice and Policy}

Despite the limitations of the present study, the findings have several practical implications. Our results suggest that interventions directed toward increasing 
individuals' confidence in their ability to overcome barriers to physical activity and positive expected outcomes of physical activity in the context of supportive workplace environments may be useful (Bandura, 2004; Dishman, DeJoy, Wilson, \& Vandenberg, 2009; Griffin-Blake \& DeJoy, 2006; Marcus, Napolitano, et al., 2007; Marcus, Williams, et al., 2006; Plotnikoff, McCargar, Wilson, \& Loucaides, 2005; Pronk, 2009; Pronk \& Kottke, 2009). As emphasized by Pronk and Kottke, activities that support individual level efforts by supportive procedures, policies, and environments are more likely to produce success on worker physical activity promotion. Golaszewski, Allen, and Edington (2008) also presented a model from the perspective of social ecology to address the need for creating supportive cultural (cultural norms, values, and climate, peer supports, etc.) and physical (facilities, awareness, services, policies, etc.) environments in workplace health promotion programs.

According to Bandura (2004), programs implemented via interactive Internetbased feedback and guidance offer a convenient way to reach a wide audience at low cost for informing, modeling, enabling, motivating, and guiding individuals to increase efforts to engage in regular physical activity. On the input side, health communications can be individually tailored to factors that affect physical activity, such as self-efficacy and outcome expectations for physical activity that can provide guidance for tailoring strategies. On the behavioral adaption side, individualized interactive feedback can increase the effectiveness of intervention efforts. Providing social support and guidance during early stages of behavior change and maintenance may enhance success in the long run (Bandura). 
However, an individually-tailored intervention to promote physical activity for IT professionals is more likely to be successful if integrated into supportive workplace environments that encourage and engage physical activity (Golaszewski et al., 2008; Marcus, Williams, et al., 2006; Pronk \& Kottke, 2009). This echoes our quantitative results that suggest supportive workplace environments promote employee physical activity, both directly and by raising their self-efficacy for physical activity. Moreover, our qualitative findings from participants' recommendations on how to promote physical activity for IT professionals also consistently point to the need for creating supportive environments that encourage and support employee physical activity. The findings can inform policy and intervention decisions. For example, workplace physical activity programs should take into account the following (arranged in descending order according to the size of the magnitude): (a) Socio-cultural environment: Along with a supportive environment, regularly offer various indoor/outdoor group physical activity programs (including families when possible) during or outside work and hold worksite exercise competitions; (b) Organizational support/policy: Provide monetary or non-monetary incentives (external rewards) and subsidies for exercise groups or fitness center membership (financial support) to motivate employees' engagement in physical activity and encourage continued participation in physical activity programs. This should be offered within a healthy work culture with less workload that does not encourage overtime so that employees have more leisure time for physical activity. Plus, issue regulations that permit time for physical activity at work and include physical activity engagement in the employee evaluation; (c) Physical environment: Offer more facilities and/or equipment for physical activity that employees can easily access in order to 
become more physically active; and (d) Education and encouragement: Provide knowledge of health risks and benefits of physical activity and information about indoor/outdoor physical activity to create the preconditions for adopting and maintaining physical activity.

The results from the analysis of our model also suggest that high job strain contributes to lower physical activity levels indirectly by reducing self-efficacy for physical activity, while working long hours contributes to higher job strain and lower physical activity levels. Therefore, interventions could increase their effectiveness by combining with components aimed at reducing the level of job strain and addressing the culture of long work hours, which may increase employees' confidence and motivation to engage in physical activity. Specifically, attempts to increase employees' physical activity may benefit from interventions that redesign jobs or reform work patterns to eliminate overtime and make work time more flexible. This could minimize job strain or mental demands and increase control over working lives. Importantly, there is evidence that redesigning jobs creates 'win-win' situations, with employers achieving business goals (e.g., improved productivity and quality, reduced absenteeism, etc.) and employees being empowered to pursue their personal goals (e.g., have more control over working patterns, maintain work-life balance, etc.) (Gryna, 2004; Karasek \& Theorell, 1990; U.K. Department Trade and Industry, 2005). Furthermore, our findings suggest that supportive workplace environments that may contribute to a pleasant work environment were negatively correlated with job strain and may be helpful for reducing excessive mental demands. 
Finally, our findings indicate that people who were more active in the past tended to maintain a physically active lifestyle. This emphasizes the importance of early intervention for establishing physical activity habits early in life that may persist into adulthood.

\section{Conclusions}

This study represents the first effort to examine the impact of work environment and psychosocial factors on physical activity of Taiwanese IT professionals, guided by a complex model using Social Cognitive Theory and incorporating variables from the demand/control model. Evidence from this study revealed that higher self-efficacy, positive outcome expectations, and supportive workplace environments increased levels of physical activity. Supportive workplace environments also contributed to higher levels of physical activity by increasing self-efficacy. Higher levels of job strain decreased levels of physical activity indirectly by reducing self-efficacy. Higher historical physical activity levels, better perceptions of health, being male, and shorter work hours also were shown to contribute to higher physical activity levels. The effect of gender on our findings was not substantial in multigroup analyses. Altogether, the quantitative findings of this study, along with the accompanying qualitative recommendations from the study participants, provide a useful foundation for employers, program developers, policy makers, and researchers in developing interventions to promote physical activity for health for IT professionals. 


\section{References}

Artazcoz, L., Cortès, I., Escribà-Agüir, V., Cascant, L., \& Villegas, R. (2009).

Understanding the relationship of long working hours with health status and health-related behaviours. Journal of Epidemiology and Community Health, 63(7), 521-527.

Bandura, A. (1986). Social foundations of thought and action: A social cognitive theory. Englewood Cliffs, N.J.: Prentice-Hall.

Bandura, A. (1997). Self-efficacy: The exercise of control. New York: W.H. Freeman.

Bandura, A. (2004). Health promotion by social cognitive means. Health Education \& Behavior, 31(2), 143-164.

Baranowski, T., Cullen, K. W., Nicklas, T., Thompson, D., \& Baranowski, J. (2003). Are current health behavioral change model helpful in guiding prevention of weight gain efforts? Obesity Research, 11 Suppl, 23S-43S.

Baranowski, T., Perry, C. L., \& Parcel, G. S. (2002). How individuals, environments, and health behavior interact: Social Cognitive Theory. In K. Glanz, B. K. Rimer \& F. M. Lewis (Eds.), Health Behavior and Health Education: Theory, Research and Practice (3rd ed., pp. 165-184). San Fransisco: Jossey-Bass.

Baron, R. M., \& Kenny, D. A. (1986). The moderator-mediator variable distinction in social psychological research: Conceptual, strategic, and statistical considerations. Journal of Personality and Social Psychology, 51(6), 1173-1182.

Baun, W. B., Bernacki, E. J., \& Tsai, S. P. (1986). A preliminary investigation: Effect of a corporate fitness program on absenteeism and health care cost. Journal of Occupational Medicine. Official Publication of the Industrial Medical Association, 28(1), 18-22.

Bentler, P. M. (2006). EQS 6 structural equations program manual. Encino, CA: Multivariate Software, Inc.

Bentler, P. M., \& Chou, C. (1987). Practical issues in structural modeling. Sociological Methods \& Research, 16(1), 78-117.

Berg, B. L. (1998). Qualitative research methods for the social sciences. Boston: Allyn and Bacon.

Bollen, K. A. (1989). Structural equations with latent variables. New York: Wiley.

Brisson, C., Larocque, B., Moisan, J., Vezina, M., \& Dagenais, G. R. (2000).

Psychosocial factors at work, smoking, sedentary behavior, and body mass index: A prevalence study among 6995 white collar workers. Journal of Occupational and Environmental Medicine, 42(1), 40-46. 
Brug J., Oenema, A., \& Ferreira, I. (2005). Theory, evidence and intervention mapping to improve behavior nutrition and physical activity interventions. International Journal of Behavioral Nutrition and Physical Activity, 2(1), 2.

Burke, M. J., Brief, A. P., \& George, J. M. (1993). The role of negative affectivity in understanding relations between self-reports of stressors and strains: A comment on the applied psychology literature. Journal of Applied Psychology, 78(3), 402412 .

Burton, W. N., McCalister, K. T., Chen, C. Y., Edington, D. W. (2005). The association of health status, worksite fitness center participation, and two measures of productivity. Journal of Occupational and Environmental Medicine, 47(4), 343351.

Burton, N. W., \& Turrell, G. (2000). Occupation, hours worked, and leisure-time physical activity. Preventive Medicine, 31(6), 673-681.

Chang, S. H. (2007). Physical activity and its determinants among workers -- $A$ case of traditional industry's employee in Taiwan. Unpublished doctoral dissertation, National Taiwan University, Taipei. (in Chinese)

Chen, C. M., \& Chang, M. (2004). Exercise behavior and related factors in career women -- The case of a bank in Taipei City. Journal of Nursing Research, 12(3), 180-190.

Chen, J. J., Wang, T. M., Lan, C. F., Huang, Y. G., Chiang, I. T., Wu, S. K. et al., (2008). Healthy lifestyle -- Physical activity. In K. N. Kuo (Ed.), Healthy People 2020 (pp.183-224). Taipei: National Health Research Institutes. (in Chinese)

Cheng, Y., Luh, W. M., \& Guo, Y. L. (2003). Reliability and validity of the Chinese version of the job content questionnaire in Taiwanese workers. International Journal of Behavioral Medicine, 10(1), 15-30.

Conroy, M. B., Cook, N. R., Manson, J. E., Buring, J. E., \& Lee, I. M. (2005). Past physical activity, current physical activity, and risk of coronary heart disease. Medicine and Science in Sports and Exercise, 37(8), 1251-1256.

Craig, C.L., Marshall, A.L., Sjostrom, M., Bauman, A. E., Booth, M. L., \& Ainsworth, B. E., et al. (2003). International physical activity questionnaire: 12-country reliability and validity. Medicine and Science in Sports and Exercise, 35(8), 13811395.

Dillman, D. A., Smyth, J. D., \& Christian, L. M. (2009). Internet, mail, and mixed-mode surveys: The tailored design method. Hoboken, NJ: Wiley \& Sons.

Dishman, R. K., DeJoy, D. M., Wilson, M. G., \& Vandenberg, R. J. (2009). Move to improve: A randomized workplace trial to increase physical activity. American Journal of Preventive Medicine, 36(2), 133-141. 
Dodson, E. A., Lovegreen, S. L., Elliott, M. B., Haire-Joshu, D., \& Brownson, R. C. (2008). Worksite policies and environments supporting physical activity in midwestern communities. American Journal of Health Promotion, 23(1), 51-55.

Duffy, M. E., Rossow, R., \& Hernandez, M. (1996). Correlates of health-promotion activities in employed Mexican American women. Nuring Research, 45(1), 18-25.

Finney, S. J., \& DiStefano, C. (2006). Non-normal and categorical data in structural equation modeling. In G. R. Hancock, \& R. O. Mueller (Eds.), Structural equation modeling: A second course. Greenwich, CT: IAP.

Flaherty J. A., Gavira, F. M., Pathak, D., Mitchell, T., Wintrob, R., Richman, J. A. et al. (1988). Developing instruments for cross-cultural psychiatric research. Journal of nervous and mental disease, 176(5), 257-263.

Gimeno, D., Elovainio, M., Jokela, M., De Vogli, R., Marmot, M. G., \& Kivimaki, M. (2009). Association between passive jobs and low levels of leisure-time physical activity: The Whitehall II cohort study. Occupational and Environmental Medicine, 66(11), 772-776.

Golaszewski, T., Allen, J., \& Edington, D. (2008). Working together to create supportive environments in worksite health promotion. American Journal of Health Promotion, 22(4), 1-10, iii.

Griffin-Blake, C. S., \& DeJoy, D. M. (2006). Evaluation of social-cognitive versus stagematched, self-help physical activity interventions at the workplace. American Journal of Health Promotion, 20(3), 200-209.

Gryna, F. M. (2004). Work overload!: Redesigning jobs to minimize stress and burnout. Milwaukee, Wis.: ASQ Quality Press.

Hamilton, M. T., Healy, G. N., Dunstan, D. W., Zderic, T. W., \& Owen, N. (2008). Too little exercise and too much sitting: Inactivity physiology and the need for new recommendations on sedentary behavior. Current Cardiovascular Risk Reports, 2(4), 292-298.

Harkness, J., Pennell, B.-E., \& Schoua-Glusberg, A. (2004). Survey questionnaire translation and assessment. In S. Presser., J. M. Rothgeb, M. P. Couper, J. T. Lessler, E. Martin, J. Martin, \& E. Singer (Eds.), Methods for Testing and Evaluating Survey Questionnaires (pp. 453-473). New York: John Wiley \& Sons, Inc.

Haskell, W. L., Lee, I. M., Pate, R. R., Powell, K. E., Blair, S. N., Franklin, B. A., et al. (2007). Physical activity and public health: Updated recommendation for adults from the American College of Sports Medicine and the American Heart Association. Circulation, 116(9), 1081-1093. 
Hellerstedt, W. L., Jeffery, R. W. (1997). The association of job strain and health behaviours in men and women. International Journal of Epidemiology, 26(3), 575-583.

Herman, C. W., Musich, S., Lu, C., Sill, S., Young, J. M., \& Edington, D. W. (2006). Effectiveness of an incentive-based online physical activity intervention on employee health status. Journal of Occupational and Environmental Medicine, 48(9), 889-895.

Hsu, J. H., \& Huang, S. L. (2001). Labour health promotion assistance plan for business health promotion (pp. I-1-I-31) (IOSH90-M341). Taipei: Institute of Occupational Safety and Health, Council of Labor Affairs. (in Chinese)

Hu, L.-t., \& Bentler, P. M. (1999). Cutoff criteria for fit indexes in covariance structure analysis: Conventional criteria versus new alternatives. Structural Equation Modeling: A Multidisciplinary Journal, 6(1), 1 - 55.

IPAQ Research Committee. (2005, November). Guidelines for data processing and analysis of the international physical activity questionnaire (IPAQ). Retrieved June 20, 2009, from http://www.ipaq.ki.se/scoring.pdf

Kaewthummanukul, T., Brown, K. C., Weaver, M. T., \& Thomas, R. R. (2006). Predictors of exercise participation in female hospital nurses. Journal of Advanced Nursing, 54(6), 663-675.

Kaleta, D., \& Jegier, A. (2005). Occupational energy expenditure and leisure-time physical activity. International Journal of Occupational Medicine and Environmental Health, 18(4), 351-356.

Kao, Y. H., Lu, C. M., \& Huang, Y. C. (2002). Impact of a transtheoretical model on the psychosocial factors affecting exercise among workers. Journal of Nursing Research, 10(4), 303-310.

Karasek, R. (1979). Job demands, job decision latitude, and mental strain: Implications for job redesign. Administrative Science Quarterly, 24(2), 285-308.

Karasek, R., Brisson, C., Kawakami, N., Houtman, I., Bongers, P., \& Amick, B. (1998). The Job Content Questionnaire (JCQ): An instrument for internationally comparative assessments of psychosocial job characteristics. Journal of Occupational Health Psychology, 3(4), 322-355.

Karasek, R., \& Theorell, T. (1990). Healthy work: Stress, productivity, and the reconstruction of working life. New York: Basic Books.

Kouvonen, A., Kivimaki, M., Elovainio, M., Virtanen, M., Linna, A., \& Vahtera, J. (2005). Job strain and leisure-time physical activity in female and male public sector employees. Preventive Medicine, 41(2), 532-539. 
Kremers, S. P., de Bruijn, G. J., Visscher, T. L., van Mechelen, W., de Vries, N. K., \& Brug, J. (2006). Environmental influences on energy balance-related behaviors: A dual-process view. International Journal of Behavioral Nutrition and Physical Activity, 3, 9 .

Kruger J, Yore, M. M., Bauer, D. R., \& Kohl, H. W. (2007). Selected barriers and incentives for worksite health promotion services and policies. American Journal of Health Promotion, 21(5), 439-447.

Ku, P. W., Fox, K. R., McKenna, J., \& Peng, T. L. (2006). Prevalence of leisure-time physical activity in Taiwanese adults: Results of four national surveys. Preventive Medicine, 43(6), 454-457.

Lallukka, T., Lahelma, E., Rahkonen, O., Roos, E., Laaksonen, E., Martikainen, P., et al. (2008). Associations of job strain and working overtime with adverse health behaviors and obesity: Evidence from the Whitehall II Study, Helsinki Health Study, and the Japanese Civil Servants Study. Social Science \& Medicine, 66(8), 1681-1698.

Lallukka, T., Sarlio-Lahteenkorva, S., Roos, E., Laaksonen, M., Rahkonen, O., \& Lahelma, E. (2004). Working conditions and health behaviours among employed women and men: The Helsinki health study. Preventive Medicine, 38(1), 48-56.

Landsbergis, P. A., Schnall, P. L., Deitz, D. K., Warren, K., Pickering, T. G., \& Schwartz, J. E. (1998). Job strain and health behaviors: Results of a prospective study. American Journal of Health Promotion, 12(4), 237-245.

Lee, H. S. (2008). The relationship between physical activity and sleep quality -- In hightech industry of $R \& D$ staff. Unpublished master's thesis, National Taiwan Normal University, Taipei. (in Chinese)

Lee, Y. S., Huang, Y. C., \& Kao, Y. H. (2005). Physical activities and correlates of clinical nurses in Taipei municipal hospitals. Journal of Nursing Research, 13(4), 281-292.

Lee, I. M., Paffenbarger, R. S., Jr., \& Hsieh, C. C. (1992). Time trends in physical activity among college alumni, 1962-1988. American Journal of Epidemiology, 135(8), 915-925.

Liou, Y. M. (2004). Development and verification of validity and reliability of the Taiwan version of international physical activity questionnaires. Unpublished doctoral dissertation, National Taiwan University, Taipei. (in Chinese)

Little, R. J. A., \& Schenker, N. (1995). Missing data. In G. Arminger, C. C. Clogg, \& M. E. Sobel (Eds.), Handbook of statistical modeling for the social and behavioral sciences (pp. 39-76). New York: Plenum Press. 
Lu, J. F., Tseng, H. M., \& Tsai, Y. J. (2003). Assessment of health-related quality of life in Taiwan (I): Development and psychometric testing of SF-36 Taiwan version. Taiwan Journal of Public Health, 10(1), 15-30. (in Chinese)

Lucove, J. C., Huston, S. L., \& Evenson, K. R. (2007). Workers' perceptions about worksite policies and environments and their association with leisure-time physical activity. American Journal of Health Promotion, 21(3), 196-200.

Lynch, W. D., Golaszewski, T. J., Clearie, A. F., Snow, D., \& Vickery, D. M. (1990). Impact of a facility-based corporate fitness program on the number of absences from work due to illness. Journal of Occupational Medicine, 32(1), 9-12.

Marcus, B. H., Napolitano, M. A., King, A. C., Lewis, B. A., Whiteley, J. A., Albrecht, A., et al. (2007). Telephone versus print delivery of an individualized motivationally tailored physical activity intervention: Project STRIDE. Health Psychology, 26(4), 401-409.

Marcus, B. H., Williams, D. M., Dubbert, P. M., Sallis, J. F., King, A. C., Yancey, A. K., et al. (2006). Physical activity intervention studies: What we know and what we need to know: A scientific statement from the American Heart Association Council on Nutrition, Physical Activity, and Metabolism (Subcommittee on Physical Activity); Council on Cardiovascular Disease in the Young; and the Interdisciplinary Working Group on Quality of Care and Outcomes Research. Circulation, 114(24), 2739-2752.

Musich, S., Adams, L., Broder, J., \& Edington, D. W. (1999). Benefits of onsite fitness. Fitness Management, 15(11), 54-57.

Nishida, Y., Suzuki, H., Wang, D. H., \& Kira, S. (2004). Psychological correlates of physical activity and exercise in Japanese male employees. International Journal of Sport and Health Science, 2, 136-144.

Norman, A., Bellocco, R., Bergstrom, A., \& Wolk, A. (2001). Validity and reproducibility of self-reported total physical activity -- Differences by relative weight. International Journal of Obesity, 25(5), 682-688.

Orsini, N., Bellocco, R., Bottai, M., Pagano, M., \& Wolk, A. (2007). Reproducibility of the past year and historical self-administered total physical activity questionnaire among older women. European Journal of Epidemiology, 22(6), 363-368.

Payne, N., Jones, F., \& Harris, P. (2002). The impact of working life on health behavior: The effect of job strain on the cognitive predictors of exercise. Journal of Occupational Health Psychology, 7(4), 342-353.

Payne, N., Jones, F., \& Harris, P. R. (2005). The impact of job strain on the predictive validity of the theory of planned behaviour: An investigation of exercise and healthy eating. British Journal of Health Psychology, 10(Pt 1), 115-131. 
Plotnikoff, R. C., McCargar, L. J., Wilson, P. M., \& Loucaides, C. A. (2005). Efficacy of an E-mail intervention for the promotion of physical activity and nutrition behavior in the workplace context. American Journal of Health Promotion, 19(6), 422-429.

Plotnikoff, R. C., Pickering, M. A., Flaman, L. M., \& Spence, J. C. (2010). The role of self-efficacy on the relationship between the workplace environment and physical activity: A longitudinal mediation analysis. Health Education \& Behavior, 37(2), 170-185.

Prodaniuk, T. R., Plotnikoff, R. C., Spence, J. C., \& Wilson, P. M. (2004). The influence of self-efficacy and outcome expectations on the relationship between perceived environment and physical activity in the workplace. International Journal of Behavioral Nutrition and Physical Activity, 1(1), 7.

Pronk, N. P. (2009). Physical activity promotion in business and industry: Evidence, context, and recommendations for a national plan. Journal of Physical Activity \& Health, 6 Suppl 2, S220-235.

Pronk, N. P., \& Kottke, T. E. (2009). Physical activity promotion as a strategic corporate priority to improve worker health and business performance. Preventive Medicine, 49(4), 316-321.

Pronk, N. P., Martinson, B., Kessler, R. C., Beck, A. L., Simon, G. E., \& Wang, P. (2004). The association between work performance and physical activity, cardiorespiratory fitness, and obesity. Journal of Occupational and Environmental Medicine, 46(1), 19-25.

Purath, J. (2006). Comparison of the traits of physically active and inactive women. Journal of the American Academy of Nurse Practitioners, 18(5), 234-240.

Rovniak, L. S., Anderson, E. S., Winett, R. A. \& Stephens, R. S. (2002). Social cognitive determinants of physical activity in young adults: A prospective structural equation analysis. Annals of Behavioral Medicine, 24(2), 149-156.

Sassen, B., Kok, G., Schaalma, H., Kiers, H., \& Vanhees, L. (2010). Cardiovascular risk profile: cross-sectional analysis of motivational determinants, physical fitness and physical activity. BMC Public Health, 10, 592.

Satorra, A., \& Bentler, P. M. (2001). A scaled difference chi-square test statistic for the moment structure analysis. Psychometrika, 66(4), 507-514.

Schneider, S., \& Becker, S. (2005). Prevalence of physical activity among the working population and correlation with work-related factors: Results from the first German national health survey. Journal of Occupational Health, 47(5), 414-423.

Shephard, R. J. (1992). A critical analysis of work-site fitness programs and their postulated economic benefits. Medicine and Science in Sports and Exercise, 24(3), 354-370. 
Shephard, R. J. (1999). Do work-site exercise and health programs work? The Physician and Sportsmedicine, 27(2), 48-72.

Spence, J. C., \& Lee, R. E. (2003). Toward a comprehensive model of physical activity. Psychology of Sport and Exercise, 4(1), 7-24.

Steinhardt, M. A., \& Dishman, R. K. (1989). Reliability and validity of expected outcomes and barriers for habitual physical activity. Journal of Occupational Medicine, 31(6), 536-546.

Taiwan 1111 Job Bank. (2007). Physically inactive group in the workplace -- A survey of physical activity habits among office workers. Retrieved February 5, 2011, from http://project.1111.com.tw/zone/pr/headline.asp?autono=1702 (in Chinese)

Taiwan Bureau of Health Promotion, Department of Health. (2007). International physical activity questionnaire Taiwan version: Long form. Retrieved June 20, 2009, from http://www.bhp.doh.gov.tw/BHPnet/Portal/Them_Show.aspx? Subject $=200712250028 \&$ Class $=2 \& \mathrm{No}=200903250002$ (in Chinese)

Taiwan Council of Labor Affairs, Executive Yuan. (2007). Career guide to industries: Computer system design services industry. Retrieved February 14, 2009, from http://statdb.cla.gov.tw/careerguide/ind/ind_detail.asp?section_id=1\&id_no=K07 2 (in Chinese)

Taiwan Science and Technology Advisory Group, Executive Yuan. (2007). Survey on supply and demand of manpower in the information technology service industry. Taipei: Author. (in Chinese)

Tavares, L. S., \& Plotnikoff, R. C. (2008). Not enough time? individual and environmental implications for workplace physical activity programming among women with and without young children. Health Care for Women International, 29(3), 244-281.

Tavares, L. S., Plotnikoff, R. C., \& Loucaides, C. (2009). Social-cognitive theories for predicting physical activity behaviours of employed women with and without young children. Psychology, Health \& Medicine, 14(2), 129-142.

U.K. Department Trade and Industry. (2005, September). Managing change: Practical ways to reduce long hours and reform working practices. Retrieved November 1 , 2010 from http://www.berr.gov.uk/files/file14239.pdf

U.S. Department of Health and Human Services. (2000). Healthy people 2010: Understanding and improving health. $2^{\text {nd }}$ ed. Washington, DC: U.S. Government Printing Office.

U.S. Department of Health and Human Services. (2008). 2008 Physical activity guidelines for Americans. Retrieved March 1, 2009 from http://www.health.gov/PAGuidelines/pdf/paguide.pdf 
Vinokur, A. D. (2005). Sturctural equation modeling (SEM). In S. J. Best \& B. Radcliff (Eds.), Polling America: An encyclopedia of public opinion (pp. 800-805). Westport, Conn.: Greenwood Press.

Wang, F., McDonald T., Champagne, L. J., \& Edington, D. W. (2004). Relationship of body mass index and physical activity to health care costs among employees. Journal of Occupational and Environmental Medicine, 46(5), 428-436.

Weisberg, H. F. (2005). The total survey error approach: A guide to the new science of survey research. Chicago: University of Chicago Press.

Williams, D. M., Anderson, E. S., \& Winett, R. A. (2005). A review of the outcome expectancy construct in physical activity research. Annals of Behavioral Medicine, 29(1), 70-79.

World Health Organization. (2002). The world health report 2002: Reducing risks, promoting healthy life. Retrieved March 18, 2008, from http://www.who.int/whr/2002/en/whr02_en.pdf

World Health Organization. (2008). Preventing noncommunicable diseases in the workplace through diet and physical activity: WHO/World Economic Forum report of a joint event. Retrieved February 1, 2009, from http://www.weforum.org/pdf/Wellness/WHOWEF_report.pdf

Yeh, W. Y., Cheng, Y., Chen, M. J., \& Chiu, W. H. (2008). Development and validation of an occupational burnout inventory. Taiwan Journal of Public Health, 27(5), 349-364. (in Chinese)

Yu, C., \& Muthén, B. (2002, April). Evaluation of the model fit indices for latent variable models with categorical and continuous outcomes. Paper presented at the annual meeting of the American Educational Research Association, New Orleans, LA. 


\title{
CHAPTER 5
}

\section{Conclusions}

\begin{abstract}
Summary
The primary purpose of this study was to examine the relationships among work environment (i.e., supportive workplace environment and job strain), psychosocial factors (i.e., outcome expectations and self-efficacy for physical activity), and physical activity in Taiwanese IT professionals. The study was conducted through the following three stages.

In the first stage, a comprehensive literature review was conducted to identify the most promising work environment and psychosocial determinants of physical activity in white-collar workers (the closest working population of IT professionals). Twenty-three studies were reviewed. Strong evidence was found for self-efficacy, perceived barriers or cons, and perceived benefit or pros. Weak evidence was found for passive jobs, high strain jobs, job control, perceived workplace environments, intention, and social support. Based on the review, a hypothesized workplace physical activity model was developed using Bandura's Social Cognitive Theory (i.e., workplace environments, outcome expectations, and self-efficacy), incorporating variables from Karasek's demand/control model (i.e., job strain; the ratio of job demands to job control). This theoretical framework was used to guide the current research. In addition, the review helped identify reliable and valid measures to be used in the present study.
\end{abstract}


In the second stage, three instruments - the Perceived Workplace Environment Scale (PWES), the Positive and Negative Outcome Expectations Scale, and the Historical Physical Activity Questionnaire originally written in English were translated into Chinese, using a modified committee approach with the consideration of cross-cultural equivalence. The translation process of the PWES is described in detail in Chapter 3.

In the third stage, a cross-sectional survey was conducted. Survey data were initially used to test the psychometric properties of the instruments used in this study. The psychometric properties of the newly translated Chinese version of the PWES (PWES-C) are presented in detail in Chapter 3. The results support the PWES-C as a reliable and valid measure of perceived workplace environment in Taiwanese IT professionals. Secondly, survey data were used to examine the relationships among work environment, psychosocial factors, and physical activity in Taiwanese IT professionals. Structural equation modeling was used to test the model. The main results showed that (a) higher physical activity levels were associated with higher scores on supportive workplace environments, positive outcome expectations, and self-efficacy for physical activity; (b) self-efficacy partially mediated the effects of supportive workplace environments on physical activity; (c) job strain had a significant indirect effect on physical activity through self-efficacy; (d) the final model accounted for $31 \%$ of the variance in physical activity; and (e) the effect of gender on our findings was not substantial in multigroup analyses.

Findings from this study are significant for several reasons. This is the first study to conduct a review of the literature on both work environment and psychosocial determinants of physical activity in white-collar workers. The results indicated a 
significant gap in the absence of exploration of the complex relationships between work environment and psychosocial factors, and their influence on physical activity among (Taiwanese) IT professionals. The review also indicated a major gap in research to use a reliable and valid instrument to measure the construct of perceived workplace physical activity environments, with the exception of one study (Prodaniuk, Plotnikoff, Spence, \& Wilson, 2004). Moreover, this is the first study to translate the English version of the PWES into Chinese and to validate the PWES-C for cross-cultural equivalence. Furthermore, this is the first study to simultaneously examine the relationships among work environment, psychosocial factors, and physical activity in Taiwanese IT professionals, using structural equation modeling.

\section{Implications}

Occupational health professionals need to be aware of workers' perceptions of workplace environments that support physical activity given its relationship to physical activity and the fact that workers spend most of their waking hours during weekdays in the workplace. A short and psychometrically sound measure of the PWES-C provides a means for occupational health professionals to effectively assess and track changes in workers' perceptions of workplace environments. The assessment helps identify the need to improve and/or create a supportive workplace environment for physical activity.

Further, the PWES-C has the potential to be used for policy decisions. For example, Taiwan government agencies with responsibility for worker health, like Council of Labor Affairs and Bureau of Health Promotion, may include this measure in a regular business evaluation associated with safety and health promotion. Only then will most 
employers devote their efforts to creating healthy environments for physical activity and promising next steps be possible.

Results from this study can inform intervention development and policy decisions aimed at meeting the goals of Taiwan Healthy People 2020. The most important components of workplace physical activity programs may be directed toward increasing individuals' confidence in overcoming barriers to engage in physical activity and positive expected outcomes of engaging in physical activity in the context of supportive environments. Specific elements of supportive workplace environments for physical activity (including socio-cultural, organizational support and policy, physical, and education and encouragement) were identified by our participants described at length in Chapter 4. Their recommendations should be taken into consideration in implementing appropriate practice and policy.

Furthermore, employers need to be aware of how job strain and long work hours can negatively impact workers' physical activity levels and perceived health. Intervention programs could maximize their effectiveness by redesigning jobs to reduce the levels of job strain and working overtime, which may increase workers' confidence and motivation to engage in physical activity. This, in turn, could have a positive impact on workers' health and productivity, and eventually leading to better business performance.

\section{Future Directions}

Future research may examine the test-retest reliability and criterion-related validity (concurrent validity) of the PWES-C, which have not been evaluated in the current study. A validated objective measure of the Workplace Physical Activity Assessment Tool (WPAAT) (Plotnikoff, Prodaniuk, Fein, \& Milton, 2005) can be used as 
a criterion measure, which is a higher-order operationalization of perceived workplace environment. However, there is no Chinese version of the WPAAT.

Future research may replicate the factor structure of the PWES-C through confirmatory factor analysis. The factor structure of the PWES-C validated in this study is considered tentative until it has been successfully replicated in different samples. The cumulative evidence from a variety of sources may suggest that the PWES-C serves as a standardized tool to measure workers' perceptions of workplace environments and changes in perceptions over time.

Theories and measures about workplace physical activity environments are still in the formulation and development stage, but their application to workplace physical activity interventions is growing. Workplace physical activity intervention research is experiencing a paradigm shift from focusing mainly on individual psychosocial factors toward emphasizing a combination of supportive workplace environments with psychosocial factors. However, more research is needed to further establish consensus on the conceptual and operational definitions and theoretical frameworks to foster the empirical examination of the concept of workplace physical activity environments.

Future research may expand to look at the outcomes of physical activity and the long term effects of physical activity both on the workers and the organization. Considering the global competition of the IT industry and the need to be efficient and effective in every piece of work, finding a way to link worker health to positive business outcomes (e.g., increased productivity and reduced absenteeism and medical costs) would create 'win-win' situations. 
Future research should continue to test the proposed theoretical framework presented in this study to determine the utility of the model in Taiwanese workers. A larger number of corporations using a longitudinal design are needed to fully explore the effects of workplace environments. Further development and expansion of this work would address the short and long term outcomes of physical activity and the potential effect of gender on model variables.

Intervention studies are needed to determine how supportive workplace environments, increased self-efficacy and positive outcome expectations can promote physical activity of Taiwanese IT professionals, and to ascertain whether it produces the intended outcomes. Reducing job strain and working overtime may be incorporated to determine the potential impact of work culture on physical activity of Taiwanese IT professionals. 


\section{References}

Plotnikoff, R. C., Prodaniuk, T. R., Fein, A. J., \& Milton, L. (2005). Development of an ecological assessment tool for a workplace physical activity program standard. Health Promotion Practice, 6(4), 453-463.

Prodaniuk, T. R., Plotnikoff, R. C., Spence, J. C., \& Wilson, P. M. (2004). The influence of self-efficacy and outcome expectations on the relationship between perceived environment and physical activity in the workplace. International Journal of Behavioral Nutrition and Physical Activity, 1(1), 7. 
APPENDICES 


\section{Information Technology Professionals Physical Activity Survey}

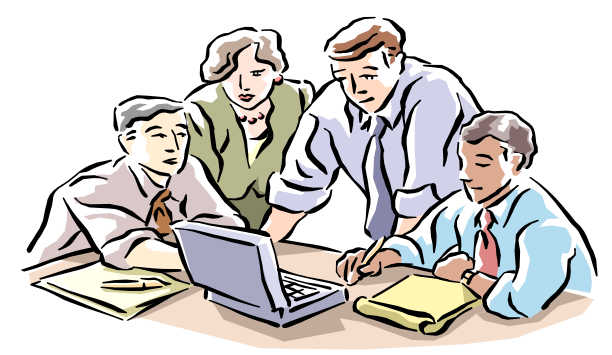

Thank you for considering this request to participate in this Physical Activity Survey of Information Technology Professionals. First, I would like to ask you three questions to see if you are eligible to participate in the study. Your answers will be kept confidential.

1. Are you under 18 years of age?
$\square$ No
Yes

2. Do you have any physical limitations or medical problems that would prevent you from performing physical activity or exercise?
$\square$ No
Yes

3. Are you currently pregnant?

$\square$ No

Yes

If you answer "Yes" to either one of these questions, please end here. I am sorry you are not eligible to participate in this study. We thank you very much for your consideration in participating in this research, and the results will be made available to the entire corporation.

Participation in this study is completely voluntary. Once you have completed the survey, please put it in the envelope (labeled with the investigator's name: Yun-Ping Lin) provided to you, seal it, and give it to your Department Assistant. The sealed envelopes will be given to the investigator.

Your answers to survey questions are very important to us. We thank you for your participation in our study!

Sincerely, Yun-Ping Lin, MSN, RN, PhD Candidate Janet Larson, PhD, RN, FAAN The University of Michigan, School of Nursing 


\section{Physical Activity}

We are interested in finding out about the kinds of physical activities that people do as part of their everyday lives. The questions will ask you about the time you spent being physically active in the last 7 days, including activities you do at work, as part of your house and yard/balcony work, to get from place to place, and in your spare time for recreation, exercise or sport. Please answer each question even if you do not consider yourself to be an active person.

In comparison with last 3 months, your physical activity during the last 7 days is (Please check)
More
As Much
Less (Please continue)

Think about all the vigorous and moderate activities that you did in the last 7 days.

Vigorous physical activities refer to activities that take hard physical effort and make you breathe much harder than normal. Count any activity that makes you work as hard as jogging and lasts at least 10 minutes at a time.

Moderate activities refer to activities that take moderate physical effort and make you breathe somewhat harder than normal. Count any activity that makes you work as hard as brisk walking and lasts at least 10 minutes at a time.

\section{Part 1: Job-Related Physical Activity}

The first section is about your work. This includes paid jobs, farming, volunteer work, course work, and any other unpaid work that you did outside your home. Do not include unpaid work you might do around your home, like housework, yard/balcony work, general maintenance, and caring for your family. These are asked in Part 3.

The next questions are about all the physical activity you did in the last $\mathbf{7}$ days as part of your paid or unpaid work. This does not include traveling to and from work.

1. During the last 7 days, on how many days did you do vigorous physical activities like heavy lifting (more than 17 taijin/10 kg), building/construction, shoveling, or climbing up stairs as part of your work? Think about only those physical activities that you did for at least 10 minutes at a time.

$$
\text { days }
$$

No vigorous job-related physical activity $\quad \longrightarrow \quad$ Skip to question 3

2. How much time did you usually spend in one day doing vigorous physical activities as part of your work? hours minutes per day 
3. Again, think about only those physical activities that you did for at least 10 minutes at a time. During the last $\mathbf{7}$ days, on how many days did you do moderate physical activities like carrying slightly heavy loads (slightly heavy means 4.5-9 kg; e.g., 2 packs of A4 paper) as part of your work? Please do not include walking while carrying light loads.

days

No moderate job-related physical activity

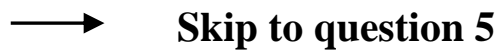

4. How much time did you usually spend in one day doing moderate physical activities as part of your work?

hours minutes per day

5. During the last $\mathbf{7}$ days, on how many days did you walk for at least 10 minutes at a time as part of your work? Please do not count any walking you did to travel to or from work. days

No job-related walking for more than 10 minutes at a time

$\longrightarrow \quad$ Skip to Part 2: Transportation

6. How much time did you usually spend in one day walking as part of your work? hours minutes per day

\section{Part 2: Transportation Physical Activity}

These questions are about how you traveled from place to place, including to places like work, stores, movies, and so on.

7. During the last $\mathbf{7}$ days, on how many days did you travel in a motor vehicle like a motorcycle, bus, car, train, or mass rapid transit (excluding bicycle)? days

$\square$ No traveling in a motor vehicle $\longrightarrow \quad$ Skip to question 9

8. How much time did you usually spend in one day traveling in a motorcycle, bus, car, train, or mass rapid transit (excluding bicycle)?

hours minutes per day

Now think only about the bicycling and walking you might have done to travel to and from work, to do errands, or to go from place to place. 
9. During the last $\mathbf{7}$ days, on how many days did you bicycle for at least 10 minutes at a time to go from place to place? days

No bicycling from place to place for at least 10 minutes at a time $\longrightarrow$ Skip to question 11

10. How much time did you usually spend in one day to bicycle from place to place?

hours minutes per day

11. During the last 7 days, on how many days did you walk for at least 10 minutes at a time to go from place to place? days

No walking from place to place for at least 10 minutes at a time $\longrightarrow$ Skip to Part 3: Housework, House Maintenance, and Caring for Family

12. How much time did you usually spend in one day walking from place to place? hours minutes per day

\section{Part 3: Housework, House Maintenance, and Caring for Family}

This section is about some of the physical activities you might have done in the last 7 days in and around your home, like housework, yard/balcony work, general maintenance work, and caring for your family.

13. Think about only those physical activities that you did for at least 10 minutes at a time. During the last 7 days, on how many days did you do vigorous physical activities like heavy lifting or digging in the garden or yard?

$$
\text { days }
$$

$\square$ No vigorous activity in garden or yard

\section{$\longrightarrow \quad$ Skip to question 15}

14. How much time did you usually spend in one day doing vigorous physical activities in the garden or yard?

hours minutes per day

15. Again, think about only those physical activities that you did for at least 10 minutes at a time. During the last 7 days, on how many days did you do moderate activities like carrying slightly heavy loads (e.g., 3 red bricks), raking, sweeping, cleaning, washing windows, and handwashing car in the garden or yard? days 
16. How much time did you usually spend in one day doing moderate physical activities in the garden or yard?

hours minutes per day

17. Once again, think about only those physical activities that you did for at least 10 minutes at a time. During the last 7 days, on how many days did you do moderate activities like carrying slightly heavy loads (e.g., grocery shopping, carrying a baby in your arms/on your back. Slightly heavy means 7.5-17 taijin/4.5-9 kg; e.g., 2 family sized milk cartons, a small watermelon, 3 unpeeled pineapples, $5 \mathrm{~kg}$ rice, 7 glass bottled Taiwan beer or rice wine, or a box of 24 canned drinks), washing windows, mopping the floor, scrubbing floors, hand washing clothes, and making the bed inside your home?

days

No moderate activity inside home

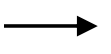

Skip to Part 4: Recreation, Sport and Leisure-Time Physical Activity

18. How much time did you usually spend in one day doing moderate physical activities inside your home?

hours minutes per day

\section{Part 4: Recreation, Sport, and Leisure-Time Physical Activity}

This section is about all the physical activities that you did in the last $\mathbf{7}$ days solely for recreation, sport, exercise or leisure. Please do not include any activities you have already mentioned.

19. Not counting any walking in your work or transportation you have already mentioned, during the last 7 days, on how many days did you walk for at least 10 minutes at a time for leisure or exercise?

$$
\text { days }
$$

No walking for leisure or exercise $\quad \longrightarrow \quad$ Skip to question 21

20. How much time did you usually spend in one day walking for leisure or exercise? hours minutes per day 
21. Think about only those physical activities that you did for at least 10 minutes at a time. During the last 7 days, on how many days did you do vigorous physical activities like jogging, continuous fast swimming, hiking, climbing stairs, aerobic dance/street dance, fast bicycling, playing ball (e.g., singles tennis, basketball, football), jumping rope, judo, tae kwon do, rock climbing, and weight training in your leisure time?

days

No vigorous activity in leisure time

$\longrightarrow \quad$ Skip to question 23

22. How much time did you usually spend in one day doing vigorous physical activities in your leisure time?

hours __ minutes per day

23. Again, think about only those physical activities that you did for at least 10 minutes at a time. During the last 7 days, on how many days did you do moderate physical activities like swimming at a regular pace, dance (e.g., bodybuilding exercise, disco, hip-hop, ballet, and folk dance; not including aerobic dance, street dance, slow dance, ballroom dance, or Yuanji dance), Tai Chi (not including Waidangong), bicycling at a regular pace, or doubles tennis, badminton, table tennis, volleyball, baseball, and softballl in your leisure time? days

No moderate activity in leisure time

$\longrightarrow \quad$ Skip to Part 5: Time Spent Sitting

24. How much time did you usually spend in one day doing moderate physical activities in your leisure time?

hours minutes per day

\section{Part 5: Time Spent Sitting}

The last questions are about the time you spend sitting while at work, at home, while doing course work and during leisure time. This may include time spent sitting at a desk, working on a computer, visiting friends, eating, reading, or sitting or lying down to watch television. Do not include any time spent sitting in a motor vehicle that you have already answered and do not count time sleeping.

25. During the last 7 days, on how many days did you work? days

26. During the last 7 days, how much time did you usually spend sitting on a weekday?

hours minutes per day

27. During the last 7 days, how much time did you usually spend sitting on a weekend day? hours minutes per day 


\section{Perceived Workplace physical activity Environment}

These questions relate to your perceptions about your workplace environment relative to physical activity. Please circle the number that best describes your response to each statement.

$\begin{array}{lccccc} & \text { None } & \text { A little } & \text { Some } & \begin{array}{c}\text { Quite a } \\ \text { lot }\end{array} & \begin{array}{c}\text { A great } \\ \text { amount }\end{array} \\ \text { 1. } \quad \begin{array}{l}\text { How much information is provided in your } \\ \text { workplace educating and/or encouraging } \\ \text { employees about physical activity? }\end{array} & 1 & 2 & 3 & 4 & 5 \\ \text { 2. } \quad \begin{array}{l}\text { Is there a positive social climate that } \\ \text { encourages physical activity in your } \\ \text { workplace? }\end{array} & 1 & 2 & 3 & 4 & 5\end{array}$
3. How much organizational capacity (i.e. infrastructure, will, and leadership) is there in your workplace that promotes physical activity for employees?

4. Has your organization used any services or resources in the community to support the physical activity of employees? (examples: local recreation/activity center, community events)
5. Does your workplace have policies that promote the physical activity of employees? (examples: no meetings scheduled over lunch, subsidies for memberships at a local fitness center or physical activity groups)

6. Are there convenient and appropriate 1 1 2 $\begin{array}{lll}3 & 4 & 5\end{array}$ facilities that you can access in order to do physical activity during the workday (including during or after working hours)? 


\section{Job Characteristics}

These questions relate to your job characteristics. Please circle the number that best describes your response to each statement.

\begin{tabular}{|c|c|c|c|c|c|}
\hline & & $\begin{array}{l}\text { Strongly } \\
\text { Disagree }\end{array}$ & Disagree & Agree & $\begin{array}{c}\text { Strongly } \\
\text { Agree }\end{array}$ \\
\hline 1. & My work requires me to do things very quickly. & 1 & 2 & 3 & 4 \\
\hline 2. & My work requires me to be very hard-working. & 1 & 2 & 3 & 4 \\
\hline$\underline{3}$. & My workload is not considered excessive. & 1 & 2 & 3 & 4 \\
\hline$\underline{4}$. & I have enough time to accomplish my work. & 1 & 2 & 3 & 4 \\
\hline 5. & $\begin{array}{l}\text { My work requires me to concentrate for a long } \\
\text { period of time. }\end{array}$ & 1 & 2 & 3 & 4 \\
\hline 6. & My work is very busy. & 1 & 2 & 3 & 4 \\
\hline 7. & My workplace has a shortage of manpower. & 1 & 2 & 3 & 4 \\
\hline 8. & My work requires me to learn new things. & 1 & 2 & 3 & 4 \\
\hline$\underline{9}$. & There are a lot of repetitive tasks in my work. & 1 & 2 & 3 & 4 \\
\hline 10. & My work requires me to be creative. & 1 & 2 & 3 & 4 \\
\hline 11. & My work allows me to make my own decisions. & 1 & 2 & 3 & 4 \\
\hline 12. & My work requires high level of skills. & 1 & 2 & 3 & 4 \\
\hline$\underline{13}$. & I don't get to decide on how I would do my work. & 1 & 2 & 3 & 4 \\
\hline 14. & I can do a variety of different things on my job. & 1 & 2 & 3 & 4 \\
\hline 15. & $\begin{array}{l}\text { On things that happen at work, my opinions are } \\
\text { influential. }\end{array}$ & 1 & 2 & 3 & 4 \\
\hline 16. & $\begin{array}{l}\text { My work provides room for me to develop my } \\
\text { own talent. }\end{array}$ & 1 & 2 & 3 & 4 \\
\hline
\end{tabular}




\section{Physical Activity Self-Efficacy}

In the next 3 months, how confident are you that you can engage in moderate or vigorous physical activity for $\mathbf{3 0}$ minutes a day (at least $\mathbf{1 0}$ minutes at a time) for at least $\mathbf{3}$ days a week in the following situations?

Moderate physical activity is any activity that is equivalent to brisk walking; vigorous physical activity is any activity that is equivalent to jogging or running.

Please circle the number that best describes your response to each statement.

\begin{tabular}{|c|c|c|c|c|c|c|c|}
\hline & & $\begin{array}{l}\text { Not At All } \\
\text { Confident }\end{array}$ & $\begin{array}{c}20 \% \\
\text { Confident }\end{array}$ & $\begin{array}{c}40 \% \\
\text { Confident }\end{array}$ & $\begin{array}{c}60 \% \\
\text { Confident }\end{array}$ & $\begin{array}{c}\mathbf{8 0} \% \\
\text { Confident }\end{array}$ & $\begin{array}{r}\text { Completely } \\
\text { Confident }\end{array}$ \\
\hline 1. & $\begin{array}{l}\text { When my family is in need of my } \\
\text { help }\end{array}$ & 1 & 2 & 3 & 4 & 5 & 6 \\
\hline 2. & $\begin{array}{l}\text { When nobody performs physical } \\
\text { activity with me }\end{array}$ & 1 & 2 & 3 & 4 & 5 & 6 \\
\hline 3. & $\begin{array}{l}\text { When nobody teaches me how to } \\
\text { do physical activity }\end{array}$ & 1 & 2 & 3 & 4 & 5 & 6 \\
\hline 4. & When I feel job stress & 1 & 2 & 3 & 4 & 5 & 6 \\
\hline 5. & When I feel that I am lacking time & 1 & 2 & 3 & 4 & 5 & 6 \\
\hline 6. & When I have a heavy workload & 1 & 2 & 3 & 4 & 5 & 6 \\
\hline 7. & When I feel a lack of energy & 1 & 2 & 3 & 4 & 5 & 6 \\
\hline 8. & When the air quality is bad & 1 & 2 & 3 & 4 & 5 & 6 \\
\hline 9. & When the weather is bad & 1 & 2 & 3 & 4 & 5 & 6 \\
\hline 10. & $\begin{array}{l}\text { When lacking adequate places for } \\
\text { physical activity }\end{array}$ & 1 & 2 & 3 & 4 & 5 & 6 \\
\hline 11. & $\begin{array}{l}\text { When lacking adequate physical } \\
\text { activity facilities or equipment }\end{array}$ & 1 & 2 & 3 & 4 & 5 & 6 \\
\hline 12. & $\begin{array}{l}\text { When lacking a budget for } \\
\text { physical activity }\end{array}$ & 1 & 2 & 3 & 4 & 5 & 6 \\
\hline 13. & When I am lazy & 1 & 2 & 3 & 4 & 5 & 6 \\
\hline 14. & $\begin{array}{l}\text { When there are other interesting } \\
\text { things to do (e.g., social activities) }\end{array}$ & 1 & 2 & 3 & 4 & 5 & 6 \\
\hline & $\begin{array}{l}\text { When nobody encourages me to } \\
\text { do physical activity }\end{array}$ & 1 & 2 & 3 & 4 & 5 & 6 \\
\hline
\end{tabular}




\section{Physical Activity Outcome Expectations}

Below is a list of possible outcomes of engaging in regular physical activity.

Regular physical activity is defined as any moderate or vigorous physical activity you performed for 30 minutes a day (at least 10 minutes at a time) for at least 3 days a week.

Moderate physical activity is any activity that is equivalent to brisk walking; vigorous physical activity is any activity that is equivalent to jogging or running.

Please rate each question twice.

- Under the heading “How Likely" please indicate how likely it is that you would experience each of the outcomes below.

- Under the heading "How Important" please indicate how much it would matter to you if each of the outcomes below occurred.

Please write one number from the following rating scale in each space.

\begin{tabular}{|lll|}
\hline How Likely & & How Important \\
\hline Not at all likely & 1 & Not at all important \\
Somewhat likely & 2 & Somewhat important \\
Moderately likely & 3 & Moderately important \\
Very likely & 4 & Very important \\
Extremely likely & 5 & Extremely important \\
\hline
\end{tabular}

SAMPLE: If it is very likely that I will build up my muscle strength, but my muscle strength is not at all important to me (i.e. it doesn't matter at all to me) then I would answer like this:
1. I will build up my muscle strength.
$\frac{\text { How Likely }}{4}$
How Important 1

If I engage in regular physical activity over the next 3 months, then:

1. I will build up my muscle strength.

2. It will be too time-consuming.

3. I will feel less depressed and/or bored.

4. I will improve my self-esteem.

5. It will make me feel tired.

6. I will not be good at doing the activity.

$\underline{7}$. It will take too long to achieve the outcomes I want.

8. I will not enjoy it.

9. I will feel less tension and stress.

\section{How Likely}

1.

2.

3.

4.

5.

6.

7.

8.

9.

- 1 .

- 3

- 4 .

5.

6.

7.

8.

9.

1.

2.

.

.

How Important

. 
Please write one number from the following rating scale in each space.

\begin{tabular}{|lll|}
\hline How Likely & & How Important \\
\hline Not at all likely & 1 & Not at all important \\
Somewhat likely & 2 & Somewhat important \\
Moderately likely & 3 & Moderately important \\
Very likely & 4 & Very important \\
Extremely likely & 5 & Extremely important \\
\hline
\end{tabular}

If I engage in regular physical activity over the next 3 months, then:

How Likely How Important

$\underline{10}$. It will be too much work and effort to motivate myself

10 .

10. to do the activity.

11. I will improve my health or reduce my risk of disease. 11

12. I will do better on my job.

12 .

11.

13. I will feel physically uncomfortable while doing the

13.

1.

12. activity (out of breath, in pain, etc.).

14. It will be difficult to find friends to do the activity with

14.

14. me.

\begin{tabular}{|c|c|c|}
\hline 15. I will feel more attractive. & 15. & 15. \\
\hline 16. I will improve my heart and lung fitness. & 16. & 16. \\
\hline$\underline{17}$. It will cost too much money. & 17. & 17. \\
\hline 18. I will find it boring. & 18. & 18. \\
\hline 19. I will increase my energy level. & 19. & 19. \\
\hline 20. I will improve my muscle tone. & 20. & 20. \\
\hline $\begin{array}{l}\text { 21. It will take away from the time I have to spend with my } \\
\text { friends. }\end{array}$ & 21. & 21. \\
\hline 22. It will take away from the time I have for my work. & 22. & 22. \\
\hline 23. I will feel better about my body. & 23. & 23. \\
\hline 24. I will gain muscle. & 24. & 24. \\
\hline 25. It will decrease the energy I have for other activities. & 25. & 25. \\
\hline
\end{tabular}




\section{Historical Physical Activity}

Think about your physical activity level at age 15, 20, and 30. Please check the one best answer for your level of physical activity for the following five activity categories at three ages (do not select multiple answers). Please refer to the example below before you answer the questions If you are under 30 years of age, please do not answer your level of physical activity at age 30.

\begin{tabular}{|l|c|c|c|}
\hline \multicolumn{2}{|l|}{ At age 15 } & At age 20 & At age 30 \\
\hline 1. Work/occupation & & & $\checkmark$ \\
\hline Mostly sitting down & $\checkmark$ & & \\
\hline Sitting down half the time & & & \\
\hline Mostly standing up & & $\checkmark$ & \\
\hline Mostly walking, lifts, carry little & & & \\
\hline Mostly walking, lifts, carry much & & & \\
\hline Heavy manual labor & & & \\
\hline
\end{tabular}

\begin{tabular}{|c|c|c|c|}
\hline & At age 15 & At age 20 & At age 30 \\
\hline \multicolumn{4}{|l|}{ 1. Work/occupation } \\
\hline \multicolumn{4}{|l|}{ Mostly sitting down } \\
\hline \multicolumn{4}{|l|}{ Sitting down half the time } \\
\hline \multicolumn{4}{|l|}{ Mostly standing up } \\
\hline \multicolumn{4}{|l|}{ Mostly walking, lifts, carry little } \\
\hline \multicolumn{4}{|l|}{ Mostly walking, lifts, carry much } \\
\hline \multicolumn{4}{|l|}{ Heavy manual labor } \\
\hline \multicolumn{4}{|l|}{ 2. Walking/bicycling } \\
\hline \multicolumn{4}{|l|}{ Hardly ever } \\
\hline \multicolumn{4}{|l|}{ Less than $20 \mathrm{~min} /$ day } \\
\hline \multicolumn{4}{|l|}{$20-40 \mathrm{~min} /$ day } \\
\hline \multicolumn{4}{|l|}{$40-60 \mathrm{~min} /$ day } \\
\hline \multicolumn{4}{|l|}{$1-1.5 \mathrm{hr} / \mathrm{day}$} \\
\hline \multicolumn{4}{|l|}{ More than $1.5 \mathrm{hr} /$ day } \\
\hline \multicolumn{4}{|l|}{ 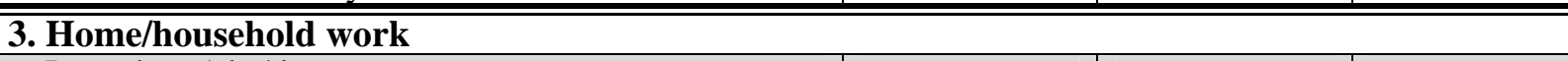 } \\
\hline \multicolumn{4}{|l|}{ Less than $1 \mathrm{hr} /$ day } \\
\hline \multicolumn{4}{|l|}{$1-2 \mathrm{hr} /$ day } \\
\hline \multicolumn{4}{|l|}{ 3-4 hr/day } \\
\hline \multicolumn{4}{|l|}{ 5-6 hr/day } \\
\hline \multicolumn{4}{|l|}{$7-8 \mathrm{hr} /$ day } \\
\hline \multicolumn{4}{|c|}{\begin{tabular}{|l|l|l} 
More than $8 \mathrm{hr} /$ day & & \\
\end{tabular}} \\
\hline \multicolumn{4}{|c|}{ 4. Leisure-time: Using computer/watching TV/reading (sitting quietly) } \\
\hline \multicolumn{4}{|c|}{\begin{tabular}{|l|l|l} 
Less than $1 \mathrm{~h} /$ day & & \\
\end{tabular}} \\
\hline \multicolumn{4}{|l|}{$1-2 \mathrm{hr} / \mathrm{day}$} \\
\hline \multicolumn{4}{|l|}{$3-4 \mathrm{hr} / \mathrm{day}$} \\
\hline \multicolumn{4}{|l|}{ 5-6 hr/day } \\
\hline \multicolumn{4}{|l|}{ More than $6 \mathrm{hr} / \mathrm{day}$} \\
\hline \multicolumn{4}{|l|}{ 5. Exercise } \\
\hline \multicolumn{4}{|l|}{ Less than $1 \mathrm{hr} /$ week } \\
\hline \multicolumn{4}{|l|}{$1 \mathrm{hr} /$ week } \\
\hline 2-3 hr/week & & & \\
\hline 4-5 hr/week & & & \\
\hline More than $5 \mathrm{hr} /$ week & & & \\
\hline $\begin{array}{l}\text { How many hours of each } 24 \text { hr day do you usually } \\
\text { sleep? }\end{array}$ & hours & hours & hours \\
\hline
\end{tabular}




\section{Background Information}

Finally, we would like to know a few things about you. Please provide the appropriate answer.

1. How old are you? YEARS OLD

2. What is your sex?

1. Male

2. Female

3. What is your highest level of education?

1. Senior high/vocational school and below

2. Junior college

3. College or university

4. Graduate school and above

4. What is your current marital status?

1. Never married

2. Married or Cohabiting

3. Separated or Divorced

4. Widowed

5. What is your job title?

6. What is your job type?

7. How long have you been employed in your current position? MONTHS YEARS

8. During the last whole week you worked (including working at home or on weekends) how many hours did you work?

HOURS PER WEEK

9. About how much do you weigh?

KG

10. About how tall are you?

CM

$\begin{array}{lcccccc} & \text { Excellent } & \begin{array}{c}\text { Very } \\ \text { Good }\end{array} & \text { Good } & \text { Fair } & \text { Poor } \\ \text { 11. In general, would you say your health is: } & 1 & 2 & 3 & 4 & 5\end{array}$




\section{Still Used to Never smoke smoke smoked}

12.

a. How would you describe your cigarette smoking habits?

$\begin{array}{lll}1 & 2 & 3\end{array}$

If not still smoke, skip to Question 13

b. If still smoke, on average, how many cigarettes did you smoke per day?

CIGARETTES PER DAY

13. How well off do you think your family is?

$\begin{array}{ccccc}\begin{array}{c}\text { Very } \\ \text { well off }\end{array} & \begin{array}{c}\text { Quite } \\ \text { well off }\end{array} & \text { Average } & \begin{array}{c}\text { Not very } \\ \text { well off }\end{array} & \begin{array}{c}\text { Not at all } \\ \text { well off }\end{array} \\ 1 & 2 & 3 & 4 & 5\end{array}$

At work At home Other places

14. Where did you complete this survey?

1

2

3

\section{Thank you for your time and participation!}

If you could make one recommendation about how to promote physical activity for information technology professionals in your company, what would it be? (Optional) 


\section{The Chinese Version of the Questionnaire}

\section{資訊科技專業人員身體活動問卷調查}

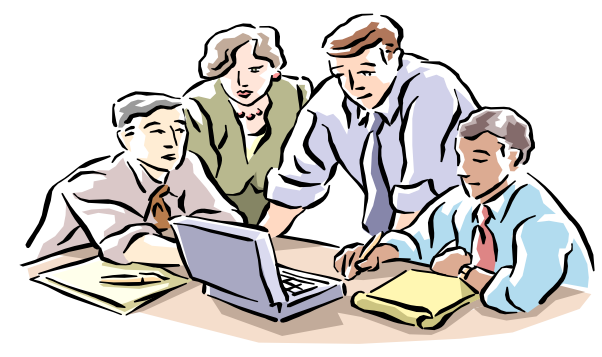

感謝您考慮填富這份『資訊科技專業人員身體活動問卷調查』! 首先想請問您三 個問題, 以確認您是否符合參與本研究的條件。您的回答會絕對保密。

\section{1. 您未滿 18 歲?}

$\square$ 否

是

2. 您現在是否有任何生理上之限制或健康問題使得您無法從事身體活動或運動?

$\square$ 否

3. 您目前是否懷孕?

$\square$ 否

若以上任一題您的回答為『是』, 請您不必填宾此問卷。我們很抱歉, 您不是本研 究所要調查的對象。我們非常感謝您考慮參與本研究, 研究結果將會提供給貴公司的 每位員工。

本研究是完全自願。一旦您填完問卷, 請將問卷放入我們所提供的信封袋裡, 信 封袋上標有研究主持人的姓名（林雲萍）, 請黏上封口, 然後將它交給您的人事小姐 $/$ 先生, 彌封的信封袋將被轉交予研究主持人。

您在問卷題目上的回答, 對本研究非常重要, 我們衷心感謝您的參與！

美國密西根大學護理學院

健康促進與風險降低科 博士候選人 林雲萍

急重症與長期照護科 教授與主任 Janet Larson 敬上 


\section{身體活動狀況}

我們想要了解民眾在日常生活中，所做身體活動的狀況。想請教您的是：

您在過去七天中花在身體活動的時間, 包括工作、做家事、整理庭院/陽台、交通, 及您在 休閒時間所做的娱樂、運動等活動中所花的時間。就算您認為自己不愛動, 也請您回答每一 個問題。

您過去七天的身體活動與過去 3 個月的身體活動比較起來（請打勾）

$\square$ 1. 比較多 $\square$ 2. 差不多 $\square$ 3. 比較少（請繼續）

回想過去七天中, 所有您做過費力及中等費力的活動。

費力的身體活動表示：這些活動會讓您的身體感覺很累，呼吸會比平常快很多。

任何一種活動它的強度跟慢跑差不多，而且一次的持續時間多於十分鐘的活動就算。

中等費力的活動表示：這些活動會讓您覺得身體有點累，呼吸會比平常快一些。

任何一種活動它的強度跟快走差不多，而且一次的持續時間多於十分鐘的活動就算。

\section{第一部份：與工作有關的身體活動}

這部份與您的工作有關, 包括您支新的工作、農務、志工、課業、以及所有在外頭不支薪的 工作。但不包括您在您家中做的不支新的工作, 如做家事、整理庭院/陽台、維修、及照顧 家人，這些會在第三部分請教您。

下面的問題是有關：過去七天中, 在支薪或不支薪的工作裡, 您所做的身體活動。但不包括 上下班的交通時間。

1. 過去七天中，您工作中有多少天，會從事費力的身體活動，如搬運重物(大於 17 台斤 $/ 10$ 公斤）、建築／營造、鏟土，或上樓梯? 但請只考慮那些一次您至少會持續 10 分鐘以上的 身體活動。

天

工作中沒有做費力的身體活動

請跳答問題 3

2. 在您的工作中, 費力的身體活動通常佔一天中的多少時間?

一天 小時 分鐘

3. 請回想那些您持續 10 分鐘以上的身體活動。過去七天, 在您的工作中, 有多少天您會做 中等費力的活動，如攜带有點重的東西走路(有點重是指 4.5 到 9 公斤：例如二包 A4 的 紙)? 請不要將提輕物的走路算進去。

天

工作中沒有做中等費力的活動

請跳答問題 5

4. 在您的工作中，中等費力的活動通常佔一天中的多少時間?

一天 小時 分鐘 
5. 在過去七天中，您工作中有多少天曾經走路持續 10 分鐘以上? 請不要將旅行或上下班的走路時間算進去。

天

工作中的走路，沒有一次持續 10 分鐘以上

一請跳答第二部分：交通

6. 在您的工作中, 走路通常佔一天中的多少時間?

一天 小時 分鐘

\section{第二部分：交通上的身體活動}

以下問題是關於您去工作、商店、電影院等這些地方的交通方式。

7. 過去七天中, 您有多少天會乘坐交通工具, 如摩托車、公車、汽車、火車或捷運（不含腳 踏車）?

天

不需乘坐交通工具 請跳答問題 9

8. 乘坐交通工具的那些天中，您通常一天花多少時間在乘坐摩托車、公車、汽車、火車或捷 運（不含腳踏車）？

一天 小時 分鐘

現在請只考虑您上下班、出差或外出的交通中，與騎腳踏車及走路相關的事。

9. 過去七天中，有幾天您以騎腳踏車當交通方式，一次至少持續 10 分鐘以上? 天

沒有以騎腳踏車當交通方式，且一次持續 10 分鐘以上

$\longrightarrow$ 請跳答 11 題

10. 您通常一天花多少時間，以騎腳踏車當交通方式?

一天 小時 分鐘

11. 過去七天中，有幾天您以走路當交通方式，而且一次至少持續 10 分鐘以上? 天

沒有以走路當交通方式，且一次持續 10 分鐘以上

$\Longrightarrow$ 請跳答第三部份：家事、 房屋維修和照顧家人

12. 您通常一天花多少時間, 以走路當交通方式?

一天 小時 分鐘 


\section{第三部份：家事、家中維修和照顧家人}

這個部份是關於過去七天中，您在住家和附近做的身體活動，如做家事、整理庭園／陽台、 一般維修和照顧您的家人。

13. 請回想那些您持續做 10 分鐘以上的身體活動。過去七天中，有幾天您在花園或庭院做費 力的活動, 像搬運重物、或鏟土?

天

沒有在花園或庭院做費力的活動

$\Longrightarrow$ 請跳答問題 15

14. 您通常一天花多少時間, 在花園或庭院做費力的身體活動?

一天 小時 分鐘

15. 請再次回想那些您持續做 10 分鐘以上的身體活動。在過去七天中，您有多少天在花園或 庭院做中等費力的活動, 像拿有點重的東西（例如三個紅碍頭）、耙土、掃地、打掃、清 洗窗户、和手工洗車?

天

沒有在花園或庭院做中等費力的活動

請跳答問題 17

16. 您通常一天花多少時間, 在花園或庭院裡, 做中等費力的活動?

一天 小時 分鐘

17. 請再次回想那些您持續 10 分鐘以上的身體活動。過去七天中, 有多少天您在家裡做中等 費力的活動，像攜带有點重的東西走路（例如買菜、背、抱小孩。有點重是指 7.5-17台 斤 /4.5-9 公斤：例如 2 瓶家庭號鮮奶、一個小玉西瓜、三個带皮鳳梨、5 公斤的米、7 瓶玻璃罐的台灣啤酒或米酒、一箱 24 瓶易開罐飲料)、清洗窗戶、拖地、擦地和手洗衣服、 鋪床。

天

沒有在家裡做中等費力的活動 請跳答第四部分：娱樂、運動和休閒的活動

18. 您通常一天花多少時間在家裡做中等費力的活動?

一天 小時 分鐘

\section{第四部分：娱樂、運動和休閒活動}

這部份是關於過去七天中, 您純粹為了娱樂、運動或休閒所做的身體活動。請不要將您前面 已經提到過的活動算進去。

19. 不要將您已經提過在工作及交通中的走路算進去。在過去七天中，您有多少天以走路當作 休閒或運動，而且一次持續 10 分鐘以上?

天

沒有以走路當作休閒或運動

請跳答問題 21 
20. 在您以走路當作休閒或運動時, 這類的走路通常佔一天中的多少時間?

一天 小時 分鐘

21. 請只考慮那些您一次至少持續做 10 分鐘以上的活動。在過去七天中，有多少天您在休閒 時, 會做費力的身體活動, 像慢跑、連續地快速游泳、上山爬坡、上樓梯、有氧舞蹈/街 舞、快速地騎腳踏車、打球（如網球單打、籃球、足球）、跳繩、柔道、跆拳道、攀岩、 重量訓練。

天

沒有在休閒時做費力的活動

請跳答問題 23

22. 您通常一天花多少時間做費力的休閒活動?

一天 小時 分鐘

23. 請再次只考慮那些您持續 10 分鐘以上的活動。過去七天内, 有多少天您在休閒時會做中等 費力的活動, 如用一般速度游泳、跳舞（如健身操、迪斯可、嘻哈舞、芭雷舞、土風舞、 民族舞蹈, 不含有氧舞蹈、街舞、慢舞、國際標準舞或元極舞)、太極（不含外丹功）、用 一般速度騎腳踏車、或是網球雙打、羽毛球、桌球、排球、棒球、壘球?

天

沒有在休閒時做中等費力的活動 $\longrightarrow$ 請跳答第五部分：坐著的時間

24. 您通常一天花多少時間, 做中等費力的休閒活動?

一天 小時 分鐘

\section{第五部分：坐著的時間}

最後的問題是關於：您在工作、居家、做功課及休閒時坐著的時間。包括坐在桌前、打電腦、 拜訪朋友、吃飯、閱讀、坐著或斜躺著看電視, 請不要包括您已經回答過的乘坐交通工具的 時間，也不要將睡著的時間算進去。

25. 過去七天中，您有幾天要工作： 天

26. 過去七天的工作天中，您一天坐著的時間有多久?

一天 小時 分鐘

27. 過去七天的假日中，您一天坐著的時間有多久?

一天 小時 分鐘 


\section{職場身體活動環境}

以下問題是關於您對職場環境有關身體活動方面的認知。對於每一句敘述, 請圈選一個最能 表達您的看法的數字。

\section{無 少許 有些 相當多 很多}

1. 您的工作場所提供了多少資訊以宣導及（或） $\quad \begin{array}{lllll}1 & 2 & 3 & 4 & 5\end{array}$ 鼓勵員工從事身體活動?

2. 您的工作場所是否具有鼓勵從事身體活動的 $\quad \begin{array}{lllll}1 & 2 & 3 & 4\end{array}$ 正面風氣?

3. 在您的工作場所中, 有多少促進員工身體活動 $\quad \begin{array}{llllll}1 & 2 & 3 & 4 & 5\end{array}$ 的組織能力（也就是基礎設施、推動意願和領 導力）?

4. 您的組織曾使用社區的任何服務或資源, 來支 $\quad \begin{array}{lllll}1 & 2 & 3 & 4 & 5\end{array}$ 持員工的身體活動嗎?（例如：當地休閒／活 動中心、社區活動）

5. 您的工作場所是否訂有鼓勵員工從事身體活 $2 \quad 3$

動的政策？（例如：不在午餐時間開會、補助 健身中心會員費或社團活動費用)

6. 是否有便利及合適的設施, 可以讓您在工作日

$2 \quad 3 \quad 4$

（包括上班或下班時間）用以從事身體活動? 


\section{工作特性}

以下敘述是關於您的工作特性。對於每一句敘述, 請圈選一個最能表達您的看法的數字。

\section{很不同意 不同意 同意 很同意}

\begin{tabular}{|c|c|c|c|c|c|}
\hline 1. & 我的工作步調很快。 & 1 & 2 & 3 & 4 \\
\hline 2. & 我的工作很辛苦。 & 1 & 2 & 3 & 4 \\
\hline 3. & 我的工作不會過量。 & 1 & 2 & 3 & 4 \\
\hline 4. & 我有足夠的時間來完成工作。 & 1 & 2 & 3 & 4 \\
\hline 5. & 我的工作會需要我長時間集中注意力。 & 1 & 2 & 3 & 4 \\
\hline 6. & 我的工作非常忙碌。 & 1 & 2 & 3 & 4 \\
\hline 7. & 我的工作場所有人力不足的現象。 & 1 & 2 & 3 & 4 \\
\hline 8. & 在工作中，我需要學習新的事物。 & 1 & 2 & 3 & 4 \\
\hline 9. & 我的工作內容, 很多是重複性的工作。 & 1 & 2 & 3 & 4 \\
\hline 10. & 在工作中，我必須具有創新的想法。 & 1 & 2 & 3 & 4 \\
\hline 11. & 在工作中，很多事我可以自己作主。 & 1 & 2 & 3 & 4 \\
\hline 12. & 我的工作需要高度的技術。 & 1 & 2 & 3 & 4 \\
\hline 13. & 對於如何執行我的工作, 我沒有什麼決定權。 & 1 & 2 & 3 & 4 \\
\hline 14. & 我的工作內容是很多元的、多樣的。 & 1 & 2 & 3 & 4 \\
\hline 15. & 對於工作上發生的事, 我的意見具有影響力。 & 1 & 2 & 3 & 4 \\
\hline 16. & 在工作中，我有機會發展自己特殊的才能。 & 1 & 2 & 3 & 4 \\
\hline
\end{tabular}




\section{身體活動自我效能}

未來三個月內, 您在以下情況中有多少把握可以從事每週至少 3 天, 每天 30 分鐘（每次至少 10 分鐘）的中等費力或費力的身體活動?

中等費力的身體活動是指相當於快走的任何活動; 費力的身體活動則是指相當於跑步（包括 慢跑）的任何活動。

對於每一句敘述, 請圈選一個最能表達您的看法的數字。

\begin{tabular}{|c|c|c|c|c|c|c|c|}
\hline 1. & 當家庭需要照顧時 & 1 & 2 & 3 & 4 & 5 & 6 \\
\hline 2. & 當缺乏從事身體活動的夥伴時 & 1 & 2 & 3 & 4 & 5 & 6 \\
\hline 3. & 當沒有人指導我從事身體活動時 & 1 & 2 & 3 & 4 & 5 & 6 \\
\hline 4. & 當感到工作壓力時 & 1 & 2 & 3 & 4 & 5 & 6 \\
\hline 5. & 當感到時間不夠用時 & 1 & 2 & 3 & 4 & 5 & 6 \\
\hline 6. & 當感到工作繁重時 & 1 & 2 & 3 & 4 & 5 & 6 \\
\hline 7. & 當感到體力不足時 & 1 & 2 & 3 & 4 & 5 & 6 \\
\hline 8. & 當感覺空氣品質不好時 & 1 & 2 & 3 & 4 & 5 & 6 \\
\hline 9. & 當天候不佳時 & 1 & 2 & 3 & 4 & 5 & 6 \\
\hline 10. & 當缺乏適當的場地從事身體活動時 & 1 & 2 & 3 & 4 & 5 & 6 \\
\hline 11. & 當缺乏適當的設備或器材從事身體活動時 & 1 & 2 & 3 & 4 & 5 & 6 \\
\hline 12. & 當缺乏經費從事身體活動時 & 1 & 2 & 3 & 4 & 5 & 6 \\
\hline 13. & 當想偷懶時 & 1 & 2 & 3 & 4 & 5 & 6 \\
\hline 14. & 當有其他事比從事身體活動更有趣時（如應酬） & 1 & 2 & 3 & 4 & 5 & 6 \\
\hline 15. & 當沒有人鼓勵我從事身體活動時 & 1 & 2 & 3 & 4 & 5 & 6 \\
\hline
\end{tabular}




\section{身體活動結果預期}

以下敘述列出了從事規律身體活動的各種可能結果。

規律的身體活動是指您從事每週 3 天，每天 30 分鐘（每次至少 10 分鐘）的中等費力或費力 的身體活動。

$\frac{\text { 中等費力的身體活動是指相當於快走的任何活動; 費力的身體活動則是指相當於跑步（包括 }}{\text { 慢跑）的任何活動。 }}$

請對以下每個敘述分別進行兩項評分。

- 在「可能程度」的標題下, 請指出您預期發生以下每個結果的可能程度。

- 在「重要程度」的標題下, 請指出如果發生以下每個結果, 對於您的重要程度。

請參考以下評分表的數字, 分別就每個敘述的可能程度及重要程度填入適當數字。

\begin{tabular}{|lll|}
\hline 可能程度 & & 重要程度 \\
\hline 完全不可能 & 1 & 完全不重要 \\
有點可能 & 2 & 有點重要 \\
頗有可能 & 3 & 頗為重要 \\
非常有可能 & 4 & 非常重要 \\
極有可能 & 5 & 極為重要 \\
\hline
\end{tabular}

範例: 假如我非常有可能強化我的肌肉力量, 可是肌肉力量對我而言完全不重要(也就是它 對我無關緊要), 那麼我的回答會像這樣:

範例 1. 我會強化我的肌肉力量 $\quad \frac{\text { 可能程度 }}{4} \quad \frac{\text { 重要程度 }}{1}$

如果我在未來三個月内從事規律的身體活動，那麼：

\section{可能程度}

重要程度

1. 我會強化我的肌肉力量

1.

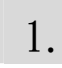

2. 從事規律的身體活動會花我太多時間。

2.

2.

3. 我會比較不憂蠜及(或) 無仰。

3.

3.

4. 我會提昇我的自尊心。

4.

5 . 從事規律的身體活動會讓我覺得很累。

5.

4.

6. 我會不太擅長於從事這種活動。

6.

7. 要達到我想要的結果會花太多時間。

7.

5.

8. 我不會樂在其中。

8.

9. 我會减少緊張和壓力

9.

6.

7.

8.

9. 
請多考以下評分表的數字, 分別就每個敘述的可能程度及重要程度填入適當數字。

\begin{tabular}{|lll|}
\hline 可能程度 & & 重要程度 \\
\hline 完全不可能 & 1 & 完全不重要 \\
有點可能 & 2 & 有點重要 \\
頗有可能 & 3 & 頗為重要 \\
非常有可能 & 4 & 非常重要 \\
極有可能 & 5 & 極為重要 \\
\hline
\end{tabular}

如果我在未夾三個月内從事規律的身體活動, 那麼：

\section{可能程度重要程度}

10. 要讓我有動機從事這種身體活動會很費功夫。

10.

10.

11. 我會改善我的健康或減少生病的風險。

11.

11.

12. 我會提高工作效率。

12.

12.

13. 當我從事身體活動時會覺得身體不舒服（喘不過

13.

13. 氣、疼痛等）。

14. 我會很難找到朋友跟我一起做活動。

14.

14.

15. 我會覺得自己更有吸引力。

15.

15.

16. 我會改善心肺功能。

16.

16.

17. 從事規律的身體活動會太花錢。

17.

17.

18. 我會覺得無聊。

18.

18.

19. 我會更有活力。

19.

19.

20. 我會增加肌肉張力（肌肉收縮緊實）。

20.

20.

21. 從事規律的身體活動會占去我和朋友相處的時間。

21.

21.

22. 從事規律的身體活動會占去我的工作時間。

22.

22.

23. 我會對自己的身體感到比較滿意。

23.

23.

24. 我會增加肌肉。

24.

24.

25. 從事規律的身體活動會减少我做其他活動的精力。

25.

25. 


\section{過去的身體活動狀況}

請回想您在 15 歲、20 歲和 30 歲那一年的身體活動程度。在下列五個活動類別中, 請針對三 個年龄分別勾選一個最能代表您身體活動程度的答案（請勿複選）。填答前請先參考以下 的工作/職業類別範例; 若您未滿 30 歲, 請您不必填富 30 歲那一年的身體活動程度。

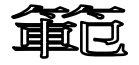

\section{1. 工作/職業 \\ 大部份的時間都坐著 有一半的時間都坐著 大部份的時間都站著 大部份的時間都在走動，不常搬運或提東西 大部份的時間都在走動，經常搬運或提東西 費力的勞動}

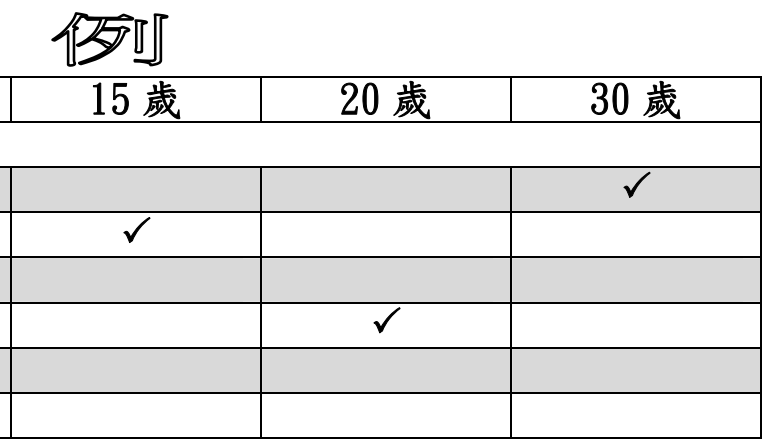

\begin{tabular}{|c|c|c|c|}
\hline & 15 歲 & 20 歲 & 30 歲 \\
\hline \multicolumn{4}{|l|}{ 1. 工作/職業 } \\
\hline \multicolumn{4}{|l|}{ 大部份的時間都坐著 } \\
\hline \multicolumn{4}{|l|}{ 有一半的時間都坐著 } \\
\hline \multicolumn{4}{|c|}{ 大部份的時間都站著 } \\
\hline \multicolumn{4}{|c|}{ 大部份的時間都在走動, 不常搬運或提東西 } \\
\hline \multicolumn{4}{|c|}{ 大部份的時間都在走動, 經常搬運或提東西 } \\
\hline \multicolumn{4}{|c|}{ 費力的勞動 } \\
\hline \multicolumn{4}{|l|}{ 2. 走路/騎腳踏車 } \\
\hline \multicolumn{4}{|l|}{ 幾乎沒有 } \\
\hline \multicolumn{4}{|l|}{ 一天不到 20 分鐘 } \\
\hline \multicolumn{4}{|l|}{ 一天 $20-40$ 分鐘 } \\
\hline \multicolumn{4}{|l|}{ 一天 $40-60$ 分鐘 } \\
\hline \multicolumn{4}{|l|}{ 一天 $1-1.5$ 小時 } \\
\hline \multicolumn{4}{|l|}{ 一天 1.5 小時以上 } \\
\hline \multicolumn{4}{|l|}{ 3. 家務/家事 } \\
\hline \multicolumn{4}{|l|}{ 一天不到 1 小時 } \\
\hline \multicolumn{4}{|l|}{ 一天 $1-2$ 小時 } \\
\hline \multicolumn{4}{|l|}{ 一天 $3-4$ 小時 } \\
\hline \multicolumn{4}{|l|}{ 一天 $5-6$ 小時 } \\
\hline \multicolumn{4}{|l|}{ 一天 $7-8$ 小時 } \\
\hline \multicolumn{4}{|l|}{ 一天 8 小時以上 } \\
\hline \multicolumn{4}{|c|}{ 44. 休閒時間：打電腦／看電視／閱讀（静坐著） } \\
\hline \multicolumn{4}{|l|}{ 一天不到 1 小時 } \\
\hline \multicolumn{4}{|l|}{ 一天 $1-2$ 小時 } \\
\hline \multicolumn{4}{|l|}{ 一天 $3-4$ 小時 } \\
\hline \multicolumn{4}{|l|}{ 一天 $5-6$ 小時 } \\
\hline \multicolumn{4}{|l|}{ 一天 6 小時以上 } \\
\hline \multicolumn{4}{|l|}{ 5. 運動 } \\
\hline \multicolumn{4}{|l|}{ 一週不到 1 小時 } \\
\hline \multicolumn{4}{|l|}{ 一週 1 小時 } \\
\hline 一週 $2-3$ 小時 & & & \\
\hline 一週 $4-5$ 小時 & & & \\
\hline 一週 5 小時以上 & & & \\
\hline 您在一天 24 小時中通常睡幾小時? & 小時 & 小時 & 小時 \\
\hline
\end{tabular}




\section{個人背景資料}

最後, 我們想對您個人的背景資料稍做瞭解, 請針對以下問題圈選或填䳆適當的答案。

1. 請問您的年齡?（請寫實足歲）

歲

2. 您的性別?
1. 男性
2. 女性

3. 您的最高學歷?
1. 高中職及以下
2. 專科
3. 大學
4. 研究所以上

4. 您目前的婚姻狀況?
1. 未婚
2. 結婚或同居
3. 分居或離婚
4. 丧偶

5. 您的職稱?

6. 您的工作類型?

7. 您受僱於目前的職位已多久? 年 月

8. 您過去一個完整的工作週內（包括平日和假日在家）實際工作總時數是多少? 一週 小時

9. 您的身高? 公分

10. 您的體重? 公斤

11. 一般來說, 您認為您目前的健康狀況是

極好的很好 好 普通 不好

$\begin{array}{lllll}1 & 2 & 3 & 4 & 5\end{array}$


12. 吸菸習慣

a. 您會如何形容自己的吸菸習慣?

還在吸菸 以前吸菸從未吸菸

123

如果答案不是「還在吸芸」, 請跳答問題 13

b. 如果還在吸菸, 您平均一天吸幾根香菸？ - 天

根香菸
13. 您認為您的家境如何?

14. 您在那裡填寫這份問卷?
很富裕相當富裕 小康 不太寬裕 很不寬裕

123

3

4

5

\section{感謝您花費寶貴的時間参與本研究！}

如果您可以向公司提供一項關於「如何增進資訊科技専業人員的身體活動」之建議, 那會是 什麼?（選擇性作答） 